\title{
A square root map on Sturmian words
}

\author{
Jarkko Peltomäki a and Markus Whiteland ${ }^{\mathrm{b}}$ \\ jspelt@utu.fi, mawhit@utu.fi \\ ${ }^{a}$ Turku Centre for Computer Science TUCS, 20520 Turku, Finland \\ a,b University of Turku, Department of Mathematics and Statistics, 20014 Turku, Finland
}

\begin{abstract}
We introduce a square root map on Sturmian words and study its properties. Given a Sturmian word of slope $\alpha$, there exists exactly six minimal squares in its language (a minimal square does not have a square as a proper prefix). A Sturmian word $s$ of slope $\alpha$ can be written as a product of these six minimal squares: $s=X_{1}^{2} X_{2}^{2} X_{3}^{2} \cdots$. The square root of $s$ is defined to be the word $\sqrt{s}=X_{1} X_{2} X_{3} \cdots$. The main result of this paper is that that $\sqrt{s}$ is also a Sturmian word of slope $\alpha$. Further, we characterize the Sturmian fixed points of the square root map, and we describe how to find the intercept of $\sqrt{s}$ and an occurrence of any prefix of $\sqrt{s}$ in $s$. Related to the square root map, we characterize the solutions of the word equation $X_{1}^{2} X_{2}^{2} \cdots X_{n}^{2}=\left(X_{1} X_{2} \cdots X_{n}\right)^{2}$ in the language of Sturmian words of slope $\alpha$ where the words $X_{i}^{2}$ are minimal squares of slope $\alpha$.

We also study the square root map in a more general setting. We explicitly construct an infinite set of non-Sturmian fixed points of the square root map. We show that the subshifts $\Omega$ generated by these words have a curious property: for all $w \in \Omega$ either $\sqrt{w} \in \Omega$ or $\sqrt{w}$ is periodic. In particular, the square root map can map an aperiodic word to a periodic word.
\end{abstract}

Keywords: sturmian word, standard word, optimal squareful word, word equation, continued fraction

\section{Introduction}

Kalle Saari studies in [16, 17] optimal squareful words which are aperiodic words containing the least number of minimal squares (that is, squares with no proper square prefixes) such that every position starts a square. Saari proves that an optimal squareful word always contains exactly six minimal squares, and he characterizes these squares; less than six minimal squares forces a word to be ultimately periodic. Moreover, he shows that Sturmian words are a proper subclass of optimal squareful words.

We propose a square root map for Sturmian words. Let $s$ be a Sturmian word of slope $\alpha$, and write it as a product of the six minimal squares in its language $\mathcal{L}(\alpha): s=X_{1}^{2} X_{2}^{2} X_{3}^{2} \cdots$. The square root of $s$ is defined to be the word $\sqrt{s}=X_{1} X_{2} X_{3} \cdots$. The main result of this paper is that the word $\sqrt{s}$ is also a Sturmian word of slope $\alpha$. More precisely, we prove that the square root of the Sturmian word $s_{x, \alpha}$ of intercept $x$ and slope $\alpha$ is $s_{\psi(x), \alpha}$ where $\psi(x)=\frac{1}{2}(x+1-\alpha)$. In addition to proving that the square root map preserves the language of a Sturmian word $s$, we show how to locate any prefix of $\sqrt{s}$ in $s$. We also characterize the Sturmian words of slope $\alpha$ which are fixed points of the square root map; they are the two Sturmian words $01 c_{\alpha}$ and $10 c_{\alpha}$ where $c_{\alpha}$ is the infinite standard Sturmian word of slope $\alpha$. The majority of the proofs of results on Sturmian words rely heavily on the interpretation of Sturmian words as rotation words. Continued fractions and results from Diophantine approximation theory play a key role in several proofs. 
Solutions of the word equation $X_{1}^{2} \cdots X_{n}^{2}=\left(X_{1} \cdots X_{n}\right)^{2}$ where the words $X_{i}^{2}$ are among the six minimal squares in $\mathcal{L}(\alpha)$ for some fixed irrational $\alpha$ are closely linked to the square root map. The study of these solutions to this word equation arises naturally from the study of fixed points of the square root map. The Sturmian fixed points of the square root map are fixed because they have arbitrarily long prefixes $X_{1}^{2} \cdots X_{n}^{2}$ which satisfy the word equation. We characterize these specific solutions, i.e., those primitive words $w$ such that $w^{2} \in \mathcal{L}(\alpha)$ and $w^{2}$ can be written as a product of minimal squares $X_{1}^{2} \cdots X_{n}^{2}$ satisfying the word equation. On the circle $[0,1)$, the interval $[w]$ of such a word $w$ can be seen to satisfy the square root condition $\psi\left(\left[w^{2}\right]\right) \subseteq[w]$, so we instead study and characterize the primitive words satisfying this square root condition. The result is that the specific solutions to the word equation (or, equivalently, the primitive words satisfying the square root condition) are the reversals of standard and semistandard words of slope $\alpha$ (see Subsection 2.3 for a definition) and the reversed standard words with the first two letters exchanged. In particular, all of these specific solutions are nonperiodic. It was known that the word equation $\left(X_{1}^{2} \cdots X_{n}^{2}\right)=X_{1}^{2} \cdots X_{n}^{2}$ has nonperiodic solutions [7], but according to our knowledge no large families of nonperiodic solutions have been identified until our result. Word equations of the type $X_{1}^{k} \cdots X_{n}^{k}=\left(X_{1} \cdots X_{n}\right)^{k}$ have been considered by Štěpán Holub $[6,7,8]$.

The final central topic of this paper concerns the square root map in a more general setting. The square root map can be defined not only for Sturmian words but for any optimal squareful word. We construct an infinite family of non-Sturmian, linearly recurrent optimal squareful words $\Gamma$ with properties similar to Sturmian words. The words $\Gamma$ are fixed points of the square root map. They are constructed by finding non-Sturmian solutions of the word equation $X_{1}^{2} \cdots X_{n}^{2}=\left(X_{1} \cdots X_{n}\right)^{2}$ and by building infinite words having arbitrarily long squares of such solutions as prefixes. The subshifts $\Omega$ generated by the words $\Gamma$ exhibit behavior similar to Sturmian subshifts. The square root map preserves the language of several but not every word in $\Omega$. Curiously, if the language of a word in $\Omega$ is not preserved under the square root map, then the image must be periodic. This result is very surprising since it is contrary to the plausible hypothesis that the square root of an aperiodic word is aperiodic.

The paper is organized as follows. In Section 3 we prove that the square root map preserves the language of a Sturmian word. As a corollary we obtain a description of those Sturmian words which are fixed points of the square root map. In Section 3 we observe that the intervals of the minimal squares in $\mathcal{L}(\alpha)$ satisfy the square root condition. In Section 4 we characterize all words $w^{2} \in \mathcal{L}(\alpha)$ satisfying the square root condition. The result is that $w^{2}$ with $w$ primitive satisfies the square root condition if and only if $w$ is a reversed standard or semistandard word or a reversed standard word with the first two letters exchanged. Section 5 contains a proof of the characterization of the specific solutions of the word equation $X_{1}^{2} \cdots X_{n}^{2}=\left(X_{1} \cdots X_{n}\right)^{2}$ mentioned earlier. We show that a primitive word $w$ satisfies the square root condition if and only if $w^{2}$ can be written as a product of minimal squares satisfying the word equation. In Section 6 we show how to locate prefixes of $\sqrt{s}$ in $s$. As an important step in proving this, we provide necessary and sufficient conditions for a Sturmian word to be a product of squares of reversed standard and semistandard words. We give a formula describing the square root of the Fibonacci word in Section 7. Section 8 is devoted to constructing the non-Sturmian fixed points $\Gamma$ mentioned above and to demonstrating that the languages of the words in their subshifts are preserved or they are mapped to periodic words. We conclude the paper by giving some remarks on possible generalizations in Section 9 and by discussing a few open problems in Section 10.

A short version of this paper was published as an extended abstract in the proceedings of WORDS 2015 [13].

\section{Notation and Preliminary Results}

In this section we review notation and basic concepts and results of word combinatorics, optimal squareful words, continued fractions, and Sturmian words. Most of the definitions and results 
provided here about words can be found in Lothaire's book [11].

An alphabet $A$ is a finite non-empty set of letters, or symbols. A (finite) word over $A$ is a finite sequence of letters of $A$ obtained by concatenation. The concatenation of two words $u=a_{0} \cdots a_{n-1}$ and $v=b_{0} \cdots b_{m-1}$ is the word $u \cdot v=u v=a_{0} \cdots a_{n-1} b_{0} \cdots b_{m-1}$. In this paper we consider only binary words, that is, words over an alphabet of size two. Most of the time we take $A$ to be the set $\{0,1\}$. The set of nonempty words over $A$ is denoted by $A^{+}$. We denote the empty word by $\varepsilon$ and set $A^{*}=A^{+} \cup\{\varepsilon\}$. A nonempty subset of $A^{*}$ is called a language. Let $w=a_{0} a_{1} \cdots a_{n-1}$ be a word of $n$ letters. We denote the length $n$ of $w$ by $|w|$; by convention $|\varepsilon|=0$. The set of proper powers of a word $w$ is denoted by $w^{+}$.

An infinite word $w$ over the alphabet $A$ is a function from the nonnegative integers to $A$. We write concisely $w=a_{0} a_{1} a_{2} \cdots$ with $a_{i} \in A$. The set of infinite words over $A$ is denoted by $A^{\omega}$. An infinite word $w$ is said to be ultimately periodic if we can write it in the form $w=u v^{\omega}=u v v v \cdots$ for some words $u, v \in A^{*}$. If $u=\varepsilon$, then $w$ is said to be periodic, or purely periodic. An infinite word which is not ultimately periodic is aperiodic. The shift operator $T$ acts on infinite words as follows: $T\left(a_{0} a_{1} a_{2} \ldots\right)=a_{1} a_{2} \cdots$.

A finite word $u$ is a factor of the finite or infinite word $w$ if we can write $w=v u z$ for some $v \in A^{*}$ and $z \in A^{*} \cup A^{\omega}$. If $v=\varepsilon$, then the factor $u$ is called a prefix of $w$. If $z=\varepsilon$, then we say that $u$ is a suffix of $w$. The set of factors of $w$, the language of $w$, is denoted by $\mathcal{L}(w)$. If $w=a_{0} a_{1} \cdots a_{n-1}$, then we let $w[i, j]=a_{i} \cdots a_{j}$ whenever the choices of positions $i$ and $j$ make sense. This notion is extended to infinite words in a natural way. An occurrence of $u$ in $w$ is a position $i$ such that $w[i, i+|u|-1]=u$. If such a position exists, then we say that $u$ occurs in $w$.

A positive integer $p$ is a period of $w=a_{0} \cdots a_{n-1}$ if $a_{i}=a_{i+p}$ for $0 \leq i \leq n-p-1$. If the finite word $w$ has period $p$ and $|w| / p \geq \alpha$ for some real $\alpha$ such that $\alpha \geq 1$, then $w$ is called an $\alpha$-repetition. An $\alpha$-repetition is minimal if it does not have an $\alpha$-repetition as a proper prefix. If $w=u^{2}$, then $w$ is a square with square root $u$. A square is minimal if it does not have a square as a proper prefix. A word $w$ is primitive if it is of the form $z^{n}$ if and only if $n=1$. Equivalently, a word $w$ is primitive if and only if $w$ occurs in $w^{2}$ exactly twice. The primitive root of $w$ is the unique primitive word $u$ such that $w=u^{n}$ for some $n \geq 1$. Let $w=v^{\omega}$ be a periodic infinite word. The minimal period of $w$ is defined to be the primitive root of $v$.

Let $w=a_{0} a_{1} \cdots a_{n-1}$ be a word. The reversal $\widetilde{w}$ of $w$ is the word $a_{n-1} \cdots a_{1} a_{0}$. If $w=\widetilde{w}$, then we call $w$ a palindrome. Let $C$ be the cyclic shift operator defined by the formula $C\left(a_{0} a_{1} \cdots a_{n-1}\right)=$ $a_{1} \cdots a_{n-1} a_{0}$. The words $w, C(w), C^{2}(w), \ldots, C^{|w|-1}(w)$ are the conjugates of $w$. If $u$ is a conjugate of $w$, then we say that $u$ is conjugate to $w$.

An infinite word $w$ is recurrent if each of its factors occurs in it infinitely often. Let $\left(i_{n}\right)_{n \geq 1}$ be the sequence of consecutive occurrences of a factor $u$ in a recurrent word $w$. The return time of $u$ is the quantity

$$
\sup \left\{i_{j+1}-i_{j}: j \in\{1,2, \ldots\}\right\},
$$

which can be infinite. The factors $w\left[i_{j}, i_{j+1}-1\right], j \geq 1$ are the returns to $u$ in $w$. If the return time of each factor of $w$ is finite, then the word $w$ is uniformly recurrent. Equivalently, $w$ is uniformly recurrent if for each factor $u$ of $w$ there exists an integer $R$ such that every factor of $w$ of length $R$ contains an occurrence of $u$. If there exists a global constant $K$ such that the return time of any factor $u$ of $w$ is at most $K|u|$, then we say that $w$ is linearly recurrent. Clearly a linearly recurrent word is uniformly recurrent. The index of a factor $u$ of an infinite word $w$ is defined to be

$$
\sup \left\{n: u^{n} \in \mathcal{L}(w)\right\} .
$$

If $w$ is uniformly recurrent and aperiodic, then the index of every factor of $w$ is finite.

A subshift $\Omega$ is a subset of $A^{\omega}$ such that

$$
\Omega=\left\{w \in A^{\omega}: \mathcal{L}(w) \subseteq \mathcal{L}\right\}
$$


for some language $\mathcal{L}$ such that $\mathcal{L} \subseteq A^{*}$. If we set above $\mathcal{L}=\mathcal{L}(w)$ where $w$ is an infinite word, then we say that the subshift $\Omega$ is generated by $w$. Subshifts are clearly shift-invariant. If every word in a subshift is aperiodic, then we call the subshift aperiodic. A subshift is minimal if it does not contain nonempty subshifts as proper subsets. A nonempty subshift is minimal if and only if it is generated by a uniformly recurrent word.

\subsection{Optimal Squareful Words}

In [17] Kalle Saari considers $\alpha$-repetitive words. An infinite word is $\alpha$-repetitive if every position in the word starts an $\alpha$-repetition and the number of distinct minimal $\alpha$-repetitions occurring in the word is finite. If $\alpha=2$, then $\alpha$-repetitive words are called squareful words. This means that every position of a squareful word begins with a minimal square. Saari proves that if the number of distinct minimal squares occurring in a squareful word is at most 5 , then the word must be ultimately periodic. On the other hand, if a squareful word contains at least 6 distinct minimal squares, then aperiodicity is possible. Saari calls the aperiodic squareful words containing exactly 6 minimal squares optimal squareful words. Further, he shows that optimal squareful words are always binary and that the six minimal squares must take a very specific form:

Proposition 2.1. Let $w$ be an optimal squareful word. If $10^{i} 1$ occurs in $w$ for some $i>1$, then the roots of the six minimal squares in $w$ are

$$
\begin{array}{ll}
S_{1}=0, & S_{4}=10^{\mathfrak{a}}, \\
S_{2}=010^{\mathfrak{a}-1}, & S_{5}=10^{\mathfrak{a}+1}\left(10^{\mathfrak{a}}\right)^{\mathfrak{b}}, \\
S_{3}=010^{\mathfrak{a}}, & S_{6}=10^{\mathfrak{a}+1}\left(10^{\mathfrak{a}}\right)^{\mathfrak{b}+1},
\end{array}
$$

for some $\mathfrak{a} \geq 1$ and $\mathfrak{b} \geq 0$.

The optimal squareful words containing the minimal square roots of (1) are called optimal squareful words with parameters $\mathfrak{a}$ and $\mathfrak{b}$. For the rest of this paper we reserve this meaning for the symbols $\mathfrak{a}$ and $\mathfrak{b}$. Furthermore, we agree that the symbols $S_{i}$ always refer to the minimal square roots (1).

Saari completely characterizes optimal squareful words [17, Theorem 17].

Proposition 2.2. An aperiodic infinite word $w$ is optimal squareful if and only if (up to renaming of letters) there exists integers $\mathfrak{a} \geq 1$ and $\mathfrak{b} \geq 0$ such that $w$ is an element of the language

$$
0^{*}\left(10^{\mathfrak{a}}\right)^{*}\left(10^{\mathfrak{a}+1}\left(10^{\mathfrak{a}}\right)^{\mathfrak{b}}+10^{\mathfrak{a}+1}\left(10^{\mathfrak{a}}\right)^{\mathfrak{b}+1}\right)^{\omega}=S_{1}^{*} S_{4}^{*}\left(S_{5}+S_{6}\right)^{\omega} .
$$

\subsection{Continued Fractions and Rational Approximations}

In this section we review results on continued fractions and best rational approximations of irrational numbers needed in this paper. Good references on these subjects are the books of Khinchin [9] and Cassels [2].

Every irrational real number $\alpha$ has a unique infinite continued fraction expansion

$$
\alpha=\left[a_{0} ; a_{1}, a_{2}, a_{3}, \ldots\right]=a_{0}+\frac{1}{a_{1}+\frac{1}{a_{2}+\frac{1}{a_{3}+\cdots}}}
$$

with $a_{0} \in \mathbb{Z}$ and $a_{k} \in \mathbb{N}$ for all $k \geq 1$. The numbers $a_{i}$ are called the partial quotients of $\alpha$. We focus here only on irrational numbers, but we note that with small tweaks much of what follows also holds for rational numbers, which have finite continued fraction expansions. 
The convergents $c_{k}=\frac{p_{k}}{q_{k}}$ of $\alpha$ are defined by the recurrences

$$
\begin{aligned}
p_{0}=a_{0}, & p_{1}=a_{1} a_{0}+1, & p_{k}=a_{k} p_{k-1}+p_{k-2}, & k \geq 2, \\
q_{0}=1, & q_{1}=a_{1}, & q_{k}=a_{k} q_{k-1}+q_{k-2}, & k \geq 2 .
\end{aligned}
$$

The sequence $\left(c_{k}\right)_{k \geq 0}$ converges to $\alpha$. Moreover, the even convergents are less than $\alpha$ and form an increasing sequence and, on the other hand, the odd convergents are greater than $\alpha$ and form a decreasing sequence.

If $k \geq 2$ and $a_{k}>1$, then between the convergents $c_{k-2}$ and $c_{k}$ there are semiconvergents (called intermediate fractions in Khinchin's book [9]) which are of the form

$$
\frac{p_{k, \ell}}{q_{k, \ell}}=\frac{\ell p_{k-1}+p_{k-2}}{\ell q_{k-1}+q_{k-2}}
$$

with $1 \leq \ell<a_{k}$. When the semiconvergents (if any) between $c_{k-2}$ and $c_{k}$ are ordered by the size of their denominators, the sequence obtained is increasing if $k$ is even and decreasing if $k$ is odd.

Note that we make a clear distinction between convergents and semiconvergents, i.e., convergents are not a specific subtype of semiconvergents.

A rational number $\frac{a}{b}$ is a best approximation of the real number $\alpha$ if for every fraction $\frac{c}{d}$ such that $\frac{c}{d} \neq \frac{a}{b}$ and $d \leq b$ it holds that

$$
|b \alpha-a|<|d \alpha-c| .
$$

In other words, any other multiple of $\alpha$ with a coefficient at most $b$ is further away from the nearest integer than $b \alpha$ is. The next important proposition shows that the best approximations of an irrational number are connected to its convergents (for a proof see Theorems 16 and 17 of [9]).

Proposition 2.3. The best rational approximations of an irrational number are exactly its convergents.

We identify the unit interval $[0,1)$ with the unit circle $\mathbb{T}$. Let $\alpha \in(0,1)$ be irrational. The map

$$
R:[0,1) \rightarrow[0,1), x \mapsto\{x+\alpha\},
$$

where $\{x\}$ stands for the fractional part of the number $x$, defines a rotation on $\mathbb{T}$. The circle partitions into the intervals $\left(0, \frac{1}{2}\right)$ and $\left(\frac{1}{2}, 1\right)$. Points in the same interval of the partition are said to be on the same side of 0 and points in different intervals are said to be on the opposite sides of 0 . (We are not interested in the location of the point $\frac{1}{2}$.) The points $\left\{q_{k} \alpha\right\}$ and $\left\{q_{k-1} \alpha\right\}$ are always on the opposite sides of 0 . The points $\left\{q_{k, \ell} \alpha\right\}$ with $0<\ell \leq a_{k}$ always lie between the points $\left\{q_{k-2} \alpha\right\}$ and $\left\{q_{k} \alpha\right\}$; see (4).

We measure the shortest distance to 0 on $\mathbb{T}$ by setting

$$
\|x\|=\min \{\{x\}, 1-\{x\}\} .
$$

We have the following facts for $k \geq 2$ and for all $l$ such that $0<l \leq a_{k}$ :

$$
\begin{aligned}
\left\|q_{k, \ell} \alpha\right\| & =(-1)^{k}\left(q_{k, \ell} \alpha-p_{k, \ell}\right), \\
\left\|q_{k, \ell} \alpha\right\| & =\left\|q_{k, \ell-1} \alpha\right\|-\left\|q_{k-1} \alpha\right\| .
\end{aligned}
$$

We can now interpret Proposition 2.3 as

$$
\min _{0<n<q_{k}}\|n \alpha\|=\left\|q_{k-1} \alpha\right\|, \quad \text { for } k \geq 1 .
$$

Note that rotating preserves distances; a fact we will often use without explicit mention. In particular, the distance between the points $\{n \alpha\}$ and $\{m \alpha\}$ is $\||n-m| \alpha\|$. Thus by (5) the minimum distance between the distinct points $\{n \alpha\}$ and $\{m \alpha\}$ with $0 \leq n, m<q_{k}$ is at least $\left\|q_{k-1} \alpha\right\|$. Formula (5) tells what is the point closest to 0 among the points $\{n \alpha\}$ for $1 \leq n \leq q_{k}-1$. We are also interested in knowing the point closest to 0 on the side opposite to $\left\{q_{k-1} \alpha\right\}$. The next result is very important and concerns this; see [12, Proposition 2.2.]. 
Proposition 2.4. Let $\alpha$ be an irrational number. Let $n$ be an integer such that $0<n<q_{k, \ell}$ with $k \geq 2$ and $0<\ell \leq a_{k}$. If $\|n \alpha\|<\left\|q_{k, \ell-1} \alpha\right\|$, then $n=m q_{k-1}$ for some integer $m$ such that $1 \leq m \leq$ $\min \left\{\ell, a_{k}-\ell+1\right\}$.

\subsection{Sturmian Words}

Sturmian words are a well-known class of infinite, aperiodic binary words with minimal factor complexity. They are defined as the infinite words having $n+1$ factors of length $n$ for every $n \geq 0$. For our purposes it is more convenient to view Sturmian words as the infinite words obtained as codings of orbits of points in an irrational circle rotation with two intervals; see [14, 11]. Let us make this more precise. The frequency $\alpha$ of letter 1 (called the slope) in a Sturmian words exists, and it is irrational. Divide the circle $\mathbb{T}$ into two intervals $I_{0}$ and $I_{1}$ defined by the points 0 and $1-\alpha$, and define the coding function $v$ by setting $v(x)=0$ if $x \in I_{0}$ and $v(x)=1$ if $x \in I_{1}$. The coding of the orbit of a point $x$ is the infinite word $s_{x, \alpha}$ obtained by setting its $n^{\text {th }}, n \geq 0$, letter to equal $v\left(R^{n}(x)\right)$ where $R$ is the rotation by angle $\alpha$. This word is Sturmian with slope $\alpha$, and conversely every Sturmian word with slope $\alpha$ is obtained this way. To make the definition proper, we need to define how $v$ behaves in the endpoints 0 and $1-\alpha$. We have two options: either take $I_{0}=[0,1-\alpha)$ and $I_{1}=[1-\alpha, 1)$ or $I_{0}=(0,1-\alpha]$ and $I_{1}=(1-\alpha, 1]$. The difference is seen in the codings of the orbits of the special points $\{-n \alpha\}$, and both options are needed to be able to obtain every Sturmian word of slope $\alpha$ as a coding of a rotation. However, in this paper we are not concerned about this choice. We make the convention that $I(x, y)$ with $x \neq y$ and $x, y \neq 0$ is either of the half-open intervals of $\mathbb{T}$ separated by the points $x$ and $y$ (taken modulo 1 if necessary) not containing the point 0 as an interior point. The interval $I(x, 0)=I(0, x)$ is either of the half-open intervals separated by the points 0 and $x$ having smallest length (the case $x=\frac{1}{2}$ is not important in this paper). Since the sequence $(\{n \alpha\})_{n \geq 0}$ is dense in $[0,1)$-as is wellknown-every Sturmian word of slope $\alpha$ has the same language (that is, the set of factors); this language is denoted by $\mathcal{L}(\alpha)$. Further, all Sturmian words are uniformly recurrent.

For every factor $w=a_{0} a_{1} \cdots a_{n-1}$ of length $n$ there exists a unique subinterval $[w]$ of $\mathbb{T}$ such that $s_{x, \alpha}$ begins with $w$ if and only if $x \in[w]$. Clearly

$$
[w]=I_{a_{0}} \cap R^{-1}\left(I_{a_{1}}\right) \cap \ldots \cap R^{-(n-1)}\left(I_{a_{n-1}}\right) .
$$

We denote the length of the interval $[w]$ by $|[w]|$. The points $0,\{-\alpha\},\{-2 \alpha\}, \ldots,\{-n \alpha\}$ partition the circle into $n+1$ intervals, which have one-to-one correspondence with the words of $\mathcal{L}(\alpha)$ of length $n$. Among these intervals the interval containing the point $\{-(n+1) \alpha\}$ corresponds to the right special factor of length $n$. A factor $w$ is right special if both $w 0, w 1 \in \mathcal{L}(\alpha)$. Similarly a factor is left special if both $0 w, 1 w \in \mathcal{L}(\alpha)$. In a Sturmian word there exists a unique right special and a unique left special factor of length $n$ for all $n \geq 0$. The language $\mathcal{L}(\alpha)$ is mirror-invariant, that is, for every $w \in \mathcal{L}(\alpha)$ also $\widetilde{w} \in \mathcal{L}(\alpha)$. It follows that the right special factor of length $n$ is the reversal of the left special factor of length $n$. Sturmian words are also balanced; that is, the number of occurrences of the letter 1 in any two factors of the same length differ at most by 1 .

Given the continued fraction expansion of an irrational $\alpha \in(0,1)$ as in (2), we define the corresponding standard sequence $\left(s_{k}\right)_{k \geq 0}$ of words by

$$
s_{-1}=1, \quad s_{0}=0, \quad s_{1}=s_{0}^{a_{1}-1} s_{-1}, \quad s_{k}=s_{k-1}^{a_{k}} s_{k-2}, \quad k \geq 2 .
$$

As $s_{k}$ is a prefix of $s_{k+1}$ for $k \geq 1$, the sequence $\left(s_{k}\right)$ converges to a unique infinite word $c_{\alpha}$ called the infinite standard Sturmian word of slope $\alpha$, and it equals $s_{\alpha, \alpha}$. Inspired by the notion of semiconvergents, we define semistandard words for $k \geq 2$ by

$$
s_{k, \ell}=s_{k-1}^{\ell} s_{k-2}
$$

with $1 \leq \ell<a_{k}$. Clearly $\left|s_{k}\right|=q_{k}$ and $\left|s_{k, \ell}\right|=q_{k, \ell}$. Instead of writing "standard or semistandard", we often simply write "(semi)standard". The set of standard words of slope $\alpha$ is denoted 
by $\operatorname{Stand}(\alpha)$, and the set of standard and semistandard words of slope $\alpha$ is denoted by $\operatorname{Stand}^{+}(\alpha)$. (Semi)standard words are left special as prefixes of the word $c_{\alpha}$. Every (semi)standard word is primitive [11, Proposition 2.2.3]. An important property of standard words is that the words $s_{k}$ and $s_{k-1}$ almost commute; namely $s_{k} s_{k-1}=w x y$ and $s_{k-1} s_{k}=w y x$ for some word $w$ and distinct letters $x$ and $y$. For more on standard words see [11,1].

The only difference between the words $c_{\alpha}$ and $c_{\bar{\alpha}}$ where $\alpha=\left[0 ; 1, a_{2}, a_{3}, \ldots\right]$ and $\bar{\alpha}=\left[0 ; a_{2}+\right.$ $\left.1, a_{3}, \ldots\right]$ is that the roles of the letters 0 and 1 are reversed. We may thus assume without loss of generality that $a_{1} \geq 2$. For the rest of this paper we make the convention that $\alpha$ stands for an irrational number in $(0,1)$ having the continued fraction expansion as in (2) with $a_{1} \geq 2$, i.e., we assume that $0<\alpha<\frac{1}{2}$. The numbers $q_{k}$ and $q_{k, \ell}$ refer to the denominators of the convergents of $\alpha$, and the words $s_{k}$ and $s_{k, \ell}$ refer to the standard or semistandard words of slope $\alpha$.

\subsection{Powers in Sturmian Words}

In this section we review some known results on powers in Sturmian words, and prove helpful results for the next section.

If a square $w^{2}$ occurs in a Sturmian word of slope $\alpha$, then the length of the word $w$ must be a really specific number, namely a denominator of a convergent or a semiconvergent of $\alpha$. The proof can be found in [3, Theorem 1] or [12, Proposition 4.1].

Proposition 2.5. If $w^{2} \in \mathcal{L}(\alpha)$ with $w$ nonempty and primitive, then $|w|=q_{0},|w|=q_{1}$ or $|w|=q_{k, \ell}$ for some $k \geq 2$ with $0<\ell \leq a_{k}$.

Next we need to know when conjugates of (semi)standard words occur as squares in a Sturmian word.

Proposition 2.6. The following holds:

(i) A factor $w \in \mathcal{L}(\alpha)$ is conjugate to $s_{k}$ for some $k \geq 0$ if and only if $|w|=\left|s_{k}\right|$ and $w^{2} \in \mathcal{L}(\alpha)$.

(ii) Let $w$ be a conjugate of $s_{k, \ell}$ with $k \geq 2$ and $0<\ell<a_{k}$. Then $w^{2} \in \mathcal{L}(\alpha)$ if and only if the intervals $[w]$ and $\left[s_{k, \ell}\right]$ have the same length.

(iii) Let $n=q_{0}, n=q_{1}$, or $n=q_{k, \ell}$ with $k \geq 2$ and $0<\ell \leq a_{k}$, and let $s$ be the (semi)standard word of length $n$. A factor $w \in \mathcal{L}(\alpha)$ of length $n$ is conjugate to $s$ if and only if $w$ and s have equally many occurrences of the letter 0 .

Proof. Claim (i) is a direct consequence of [3, Theorem 3] or alternatively [12, Theorem 4.5]. Claim (ii) can be inferred from Theorems 4.3 and 4.5 of [12]. Finally, claim (iii) is evident from the proof of [12, Theorem 4.3], but a short proof can be given: the idea is that every factor of length $n$ except one exceptional factor $v$ is conjugate to $s$ since $s^{2}$ occurs in $\mathcal{L}(\alpha)$ by $(i)$ and (ii). As not every factor of length $n$ may have the same number of letters 0 (a right special factor always extends to two factors having different number of letters 0 ), it must be that $v$ has a different number of letters 0 than any conjugate of $s$.

We also need to know the index of certain factors of Sturmian words. The following proposition follows directly from Theorems 3 and 4 of [3] or from [12, Theorem 4.5].

Proposition 2.7. The index of the standard word $s_{k}$ in $\mathcal{L}(\alpha)$ is $a_{k+1}+2$ for $k \geq 2$ and $a_{2}+1$ for $k=1$. The index of the semistandard word $s_{k, \ell}$ in $\mathcal{L}(\alpha)$ with $k \geq 2$ and $0<\ell<a_{k}$ is 2 . 


\section{The Square Root Map}

In [17] Saari observed that every Sturmian word with slope $\alpha=\left[0 ; a_{1}, a_{2}, \ldots\right]$ is an optimal squareful word with parameters $\mathfrak{a}=a_{1}-1$ and $\mathfrak{b}=a_{2}-1$. The assumption $0<\alpha<\frac{1}{2}$ implies that $0^{2} \in \mathcal{L}(\alpha)$, so the six minimal squares in $\mathcal{L}(\alpha)$ are the same as in (1). In particular, Saari's result means that every Sturmian word can be (uniquely) written as a product of the six minimal squares of slope $\alpha$ (1). Thus the square root map introduced next is well-defined.

Definition 3.1. Let $s$ be a Sturmian word with slope $\alpha$ and factorize it as a product of minimal squares $s=X_{1}^{2} X_{2}^{2} X_{3}^{2} \cdots$. The square root of $s$ is then defined to be the word $\sqrt{s}=X_{1} X_{2} X_{3} \cdots$.

Let us consider as an example the famous Fibonacci word $f$. The Fibonacci word is a Sturmian word of slope $[0 ; 2,1,1, \ldots]$, so it has parameters $\mathfrak{a}=1$ and $\mathfrak{b}=0$. It is also the fixed point of the substitution $0 \mapsto 01,1 \mapsto 0$. For more information, see for instance [11]. We have that

$$
\begin{aligned}
f & =(010)^{2}(100)^{2}(10)^{2}(01)^{2} 0^{2}(10010)^{2}(01)^{2} \cdots \text { and } \\
\sqrt{f} & =010 \cdot 100 \cdot 10 \cdot 01 \cdot 0 \cdot 10010 \cdot 01 \cdots .
\end{aligned}
$$

Note that a square root map can be defined for any optimal squareful word. However, now we only focus on Sturmian words; we study later the square root map for other optimal squareful words in Section 8.

We aim to prove the surprising fact that given a Sturmian word $s$ the word $\sqrt{s}$ is also a Sturmian word having the same slope as $s$. Moreover, knowing the intercept of $s$, we can compute the intercept of $\sqrt{s}$.

In the proof we need a special function $\psi: \mathbb{T} \rightarrow \mathbb{T}$ defined as follows. For $x \in(0,1)$ we set

$$
\psi(x)=\frac{1}{2}(x+1-\alpha)
$$

and we set

$$
\psi(0)= \begin{cases}\frac{1}{2}(1-\alpha), & \text { if } I_{0}=[0,1-\alpha), \\ 1-\frac{\alpha}{2}, & \text { if } I_{0}=(0,1-\alpha] .\end{cases}
$$

The mapping $\psi$ moves a point $x$ on the circle $\mathbb{T}$ towards the point $1-\alpha$ by halving the distance between the points $x$ and $1-\alpha$. The distance to $1-\alpha$ is measured in the interval $I_{0}$ or $I_{1}$ depending on which of these intervals the point $x$ belongs to.

We can now state the result.

Theorem 3.2. Let $s_{x, \alpha}$ be a Sturmian word with slope $\alpha$ and intercept $x$. Then $\sqrt{s_{x, \alpha}}=s_{\psi(x), \alpha}$. In particular, $\sqrt{s_{x, \alpha}}$ is a Sturmian word with slope $\alpha$.

For a combinatorial version of the above theorem see Theorem 6.5 in Section 6.

The main idea of the proof is to demonstrate that the square root map is actually the symbolic counterpart of the function $\psi$. We begin with a definition.

Definition 3.3. A square $w^{2} \in \mathcal{L}(\alpha)$ satisfies the square root condition if $\psi\left(\left[w^{2}\right]\right) \subseteq[w]$.

Note that if the interval $[w]$ in the above definition has $1-\alpha$ as an endpoint, then $w$ automatically satisfies the square root condition. This is because $\psi$ moves points towards the point $1-\alpha$ but does not map them over this point. Actually, if $w$ satisfies the square root condition, then necessarily the interval $[w]$ has $1-\alpha$ as an endpoint (see Corollary 4.3 ).

We will only sketch the proof of the following lemma.

Lemma 3.4. For every $i \in\{1, \ldots, 6\}$ the minimal square root $S_{i}$ of slope $\alpha$ satisfies the square root condition and $\psi\left(\left\{x+2\left|S_{i}\right| \alpha\right\}\right)=\left\{\psi(x)+\left|S_{i}\right| \alpha\right\}$ for all $x \in\left[S_{i}^{2}\right]$. 


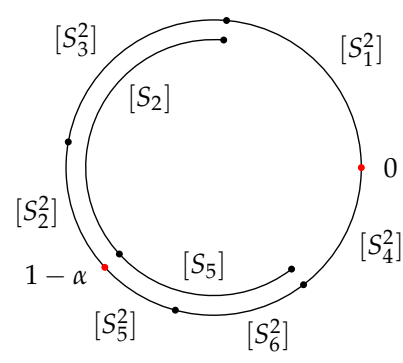

Figure 1: The positions of the intervals on the circle in the proof sketch of Lemma 3.4.

Proof Sketch. It is straightforward to verify that

$$
\begin{array}{ll}
{\left[S_{1}\right]=I(0,1-\alpha),} & {\left[S_{4}\right]=I(1-\alpha, 1),} \\
{\left[S_{2}\right]=I(-2 \alpha, 1-\alpha),} & {\left[S_{5}\right]=I\left(1-\alpha,-q_{2,1} \alpha\right),} \\
{\left[S_{3}\right]=I(-2 \alpha, 1-\alpha),} & {\left[S_{6}\right]=I\left(1-\alpha,-q_{2,1} \alpha\right)}
\end{array}
$$

and

$$
\begin{array}{ll}
{\left[S_{1}^{2}\right]=I(0,-2 \alpha),} & {\left[S_{4}^{2}\right]=I\left(-q_{2,1} \alpha, 1\right),} \\
{\left[S_{2}^{2}\right]=I\left(-\left(q_{2,1}+1\right) \alpha, 1-\alpha\right),} & {\left[S_{5}^{2}\right]=I\left(1-\alpha,-\left(q_{3,1}+1\right) \alpha\right),} \\
{\left[S_{3}^{2}\right]=I\left(-2 \alpha,-\left(q_{2,1}+1\right) \alpha\right),} & {\left[S_{6}^{2}\right]=I\left(-\left(q_{3,1}+1\right) \alpha,-q_{2,1} \alpha\right),}
\end{array}
$$

see Figure 1 . Since $\psi$ does not map points over the point $1-\alpha$, it is evident that every minimal square root satisfies the square root condition.

Consider then the latter claim. Let $i \in\{1, \ldots, 6\}$. Suppose that $x \in\left[S_{i}^{2}\right] \backslash\{0\},\left\{x+2\left|S_{i}\right| \alpha\right\} \neq$ 0 , and $\left\lfloor x+2\left|S_{i}\right| \alpha\right\rfloor=2 r$ for some $r \geq 0$. Then

$$
\psi\left(\left\{x+2\left|S_{i}\right| \alpha\right\}\right)=\frac{1}{2}\left(x+2\left|S_{i}\right| \alpha-2 r+1-\alpha\right)=\psi(x)+\left|S_{i}\right| \alpha-r=\left\{\psi(x)+\left|S_{i}\right| \alpha\right\}
$$

since $\psi$ is a function from $\mathbb{T}$ to $\mathbb{T}$. We consider next the cases $i=1$ and $i=5$; the other cases are similar.

Suppose that $S_{i}=S_{1}$. Now $x+2 \alpha \geq 2 \alpha>0=2 p_{0}$ and $x+2 \alpha \leq 1-2 \alpha+2 \alpha=1=2 p_{0}+1$, so $x+2 \alpha \in\left(2 p_{0}, 2 p_{0}+1\right]$. The claim is thus clear as in (6) if $x \neq 0$ and $x \neq 1-2 \alpha$. If $x=0$, then $I_{0}=[0,1-\alpha)$ and $\{\psi(x)+\alpha\}=\left\{\frac{1}{2}(1-\alpha)+\alpha\right\}=\frac{1}{2}(1+\alpha)=\psi(\{x+2 \alpha\})$. If $x=1-2 \alpha$, then $I_{0}=(0,1-\alpha]$ and $\psi(\{x+2 \alpha\})=1-\frac{\alpha}{2}=\{\psi(x)+\alpha\}$.

Assume then that $S_{i}=S_{5}$. Note that $\left|S_{5}\right|=q_{2}$. Using (4) we obtain that

$$
\begin{aligned}
x+2 q_{2} \alpha & \leq\left\|\left(q_{3,1}+1\right) \alpha\right\|+2 q_{2} \alpha \\
& =1-\alpha+\left\|q_{3,1} \alpha\right\|+2 p_{2}+2\left\|q_{2} \alpha\right\| \\
& =1-\alpha+\left\|q_{1} \alpha\right\|-\left\|q_{2} \alpha\right\|+2 p_{2}+2\left\|q_{2} \alpha\right\| \\
& =1-\alpha+\left\|q_{1} \alpha\right\|+\left\|q_{2} \alpha\right\|+2 p_{2} \\
& \leq 2 p_{2}+1,
\end{aligned}
$$

where equality holds only if $x=\left\|\left(q_{3,1}+1\right) \alpha\right\|$ and $a_{2}=1$. The length of the interval $\left[S_{5}^{2}\right]$ is $\left\|q_{3,1} \alpha\right\|$. Since $1-\alpha \geq \alpha+\left\|q_{1} \alpha\right\|$ and $\alpha>\left\|q_{1} \alpha\right\|>\left\|q_{2} \alpha\right\|$, it follows from the preceding inequalities that $x+2 q_{2} \alpha>2 p_{2}$. Therefore $x+2 q_{2} \alpha \in\left(2 p_{2}, 2 p_{2}+1\right]$. If $a_{2}>1$ or $x \neq\left\|\left(q_{3,1}+1\right) \alpha\right\|$, then the conclusion follows as in (6). Suppose finally that $a_{2}=1$ and $x=\left\|\left(q_{3,1}+1\right) \alpha\right\|$. Now 
$I_{0}=(0,1-\alpha]$, so $\psi\left(\left\{x+2 q_{2} \alpha\right\}\right)=\psi(0)=1-\frac{\alpha}{2}$. On the other hand,

$$
\begin{aligned}
\psi(x)+q_{2} \alpha & =\frac{1}{2}\left(1-\alpha+\left\|q_{3,1} \alpha\right\|+1-\alpha\right)+p_{2}+\left\|q_{2} \alpha\right\| \\
& =\frac{1}{2}\left(1-\alpha+\left\|q_{1} \alpha\right\|-\left\|q_{2} \alpha\right\|+1-\alpha+2\left\|q_{2} \alpha\right\|\right)+p_{2} \\
& =1-\frac{\alpha}{2}+p_{2}
\end{aligned}
$$

so the conclusion holds also in this case.

Proof of Theorem 3.2. Write $s_{x, \alpha}=X_{1}^{2} X_{2}^{2} X_{3}^{2} \cdots$ as a product of minimal squares. Since the minimal square $X_{1}^{2}$ satisfies the square root condition by Lemma 3.4, we have that $\psi(x) \in\left[X_{1}\right]$. Hence both $\sqrt{s_{x, \alpha}}$ and $s_{\psi(x), \alpha}$ begin with $X_{1}$. Lemma 3.4 implies that $\psi\left(\left\{x+2\left|X_{1}\right| \alpha\right\}\right)=\left\{\psi(x)+\left|X_{1}\right| \alpha\right\}$ for all $x \in\left[X_{1}^{2}\right]$. Thus by shifting $s_{x, \alpha}$ the amount $2\left|X_{1}\right|$ and by applying the preceding reasoning, we conclude that $s_{\psi(x), \alpha}$ shifted by the amount $\left|X_{1}\right|$ begins with $X_{2}$. Therefore the words $\sqrt{s_{x, \alpha}}$ and $s_{\psi(x), \alpha}$ agree on their first $\left|X_{1}\right|+\left|X_{2}\right|$ letters. By repeating this procedure, we conclude that $\sqrt{s_{x, \alpha}}=s_{\psi(x), \alpha}$.

Theorem 3.2 allows us to effortlessly characterize the Sturmian words which are fixed points of the square root map.

Corollary 3.5. The only Sturmian words of slope $\alpha$ which are fixed by the square root map are the two words $01 c_{\alpha}$ and $10 c_{\alpha}$, both having intercept $1-\alpha$.

Proof. The only fixed point of the map $\psi$ is the point $1-\alpha$. Having this point as an intercept, we obtain two Sturmian words: either $01 c_{\alpha}$ or $10 c_{\alpha}$, depending on which of the intervals $I_{0}$ and $I_{1}$ the point $1-\alpha$ belongs to.

The set $\left\{01 c_{\alpha}, 10 c_{\alpha}\right\}$ is not only the set of fixed points but also the unique attractor of the square root map in the set of Sturmian words of slope $\alpha$. When iterating the square root map on a fixed Sturmian word $s_{x, \alpha}$, the obtained word has longer and longer prefixes in common with either of the words $01 c_{\alpha}$ and $10 c_{\alpha}$ because $\psi^{n}(x)$ tends to $1-\alpha$ as $n$ increases.

\section{One Characterization of Words Satisfying the Square Root Condition}

In the previous section we saw that the minimal squares, which satisfy the square root condition, were crucial in proving that the square root of a Sturmian word is again Sturmian with the same slope. The minimal squares of slope $\alpha$ are not the only squares in $\mathcal{L}(\alpha)$ satisfying the square root condition; in this section we will characterize combinatorially such squares. To be able to state the characterization, we need to define

$$
\operatorname{RStand}(\alpha)=\{\widetilde{w}: w \in \operatorname{Stand}(\alpha)\},
$$

the set of reversed standard words of slope $\alpha$. Similarly we set

$$
\operatorname{RStand}^{+}(\alpha)=\left\{\widetilde{w}: w \in \operatorname{Stand}^{+}(\alpha)\right\} .
$$

We also need the operation $L$ which exchanges the first two letters of a word (we do not apply this operation to too short words).

The main result of this section is the following.

Theorem 4.1. A square $w^{2} \in \mathcal{L}(\alpha)$ satisfies the square root condition if and only if $w \in R \operatorname{Rtand}^{+}(\alpha) \cup$ $L(R \operatorname{Stand}(\alpha))$. 
As we remarked in Section 3, a square $w^{2} \in \mathcal{L}(\alpha)$ trivially satisfies the square root condition if its interval $[w]$ has $1-\alpha$ as an endpoint. Our aim is to prove that the converse is also true. We begin with a technical lemma.

Lemma 4.2. Let $n=q_{1}$ or $n=q_{k, l}$ for some $k \geq 2$ with $0<l \leq a_{k}$, and let $i$ be an integer such that $1<i \leq n$.

(i) If $\{-i \alpha\} \in I_{0}$ and $\{-(i+n) \alpha\}<\{-i \alpha\}$, then $\psi(-(i+n) \alpha)>\{-i \alpha\}$.

(ii) If $\{-i \alpha\} \in I_{1}$ and $\{-(i+n) \alpha\}>\{-i \alpha\}$, then $\psi(-(i+n) \alpha)<\{-i \alpha\}$.

Proof. We prove (i), the second assertion is symmetric. Suppose $\{-i \alpha\} \in I_{0}$ and $\{-(i+n) \alpha\}<$ $\{-i \alpha\}$. Note that the distance between the points $\{-i \alpha\}$ and $\{-(i+n) \alpha\}$ is less than $\alpha$. It follows that $\{-n \alpha\} \in I_{1}$. Assume on the contrary that $\psi(-(i+n) \alpha) \leq\{-i \alpha\}$, that is,

$$
\{-(i+n) \alpha\}+\frac{1}{2}(\{1-\alpha\}-\{-(i+n) \alpha\}) \leq\{-i \alpha\} .
$$

Since $0<\{-(i+n) \alpha\}<\{-i \alpha\}$, the distance between $\{-(i+n) \alpha\}$ and $\{-i \alpha\}$ is the same as the distance between 1 and $\{-n \alpha\}$. Thus by substituting $\{-(i+n) \alpha\}=\{-i \alpha\}-(1-\{-n \alpha\})$ to the above and rearranging, we have that

$$
\{1-\alpha\}-\{-i \alpha\} \leq 1-\{-n \alpha\} .
$$

Since $\{-n \alpha\} \in I_{1}$, we obtain that

$$
\|-(i-1) \alpha\| \leq\|-n \alpha\| .
$$

Suppose now first that $n=q_{k, l}$ for some $k \geq 2$ and $0<l \leq a_{k}$. Since $i-1<n$, Proposition 2.4 and (7) imply that $i-1=m q_{k-1}$ for some $1 \leq m \leq \min \left\{l, a_{k}-l+1\right\}$. As $\{-n \alpha\} \in I_{1}$, the point $\left\{-q_{k-1} \alpha\right\}$ must lie on the opposite side of 0 in the interval $I_{0}$. Therefore $\{-(i-1) \alpha\} \in I_{0}$. Then by (7), the point $\{-i \alpha\}$ must lie in $I_{1}$. This is a contradiction. Suppose then that $n=q_{1}$. It is easy to see that (7) cannot hold for any $i$ greater than 1 . This concludes the proof.

Corollary 4.3. If $w^{2} \in \mathcal{L}(\alpha)$ with $w$ primitive satisfies the square root condition, then the interval [w] has $1-\alpha$ as an endpoint.

Proof. Let $n=|w|$. Proposition 2.5 implies that $n=q_{0}, n=q_{1}$, or $n=q_{k, l}$ for some $k \geq 2$ with $0<l \leq a_{k}$. Say $n=q_{0}=1$. As the only factor of length 1 occurring as a square is 0 , the claim holds as $[0]=I_{0}=I(0,1-\alpha)$. Suppose then that $n=q_{1}$ or $n=q_{k, l}$.

Let $[w]=I(-i \alpha,-j \alpha)$. Then either $\left[w^{2}\right]=I(-i \alpha,-(j+|w|) \alpha)$ or $\left[w^{2}\right]=I(-(i+|w|) \alpha,-j \alpha)$. Suppose first that $[w] \subseteq I_{0}$. By symmetry we may assume that $\{-j \alpha\}>\{-i \alpha\}$. Now $\left[w^{2}\right]=$ $[-(i+|w|) \alpha,-j \alpha)$ if and only if $j=1$. Namely, if $j \neq 1$, then it is clear that it is possible to find a point $x \in I(-i \alpha,-j \alpha)$ close to $\{-j \alpha\}$ such that $\psi(x)>\{-j \alpha\}$, so the condition $\psi\left(\left[w^{2}\right]\right) \subseteq[w]$ cannot be satisfied. If $\left[w^{2}\right]=[-i \alpha,-(j+|w|) \alpha)$ and $j \neq 1$, then by Lemma $4.2 \psi(-(j+|w|) \alpha)>$ $\{-j \alpha\}$, so the condition $\psi\left(\left[w^{2}\right]\right) \subseteq[w]$ cannot be satisfied. Thus also in this case necessarily $j=1$. The case where $[w] \subseteq I_{1}$ is proven symmetrically using the latter symmetric assertion of Lemma 4.2.

Next we study in more detail the properties of squares $w^{2} \in \mathcal{L}(\alpha)$ whose interval has $1-\alpha$ as an endpoint.

Proposition 4.4. Consider the intervals of factors in $\mathcal{L}(\alpha)$ of length $n=q_{1}$ or $n=q_{k, l}$ with $k \geq 2$ and $0<l \leq a_{k}$. Let $u$ and $v$ be the two distinct words of length $n$ having intervals with endpoint $1-\alpha$. Then the following holds.

(i) There exists a word $w$ such that $u=x y w$ and $v=y x w=L(u)$ for distinct letters $x$ and $y$. 
(ii) Either $u$ or $v$ is right special.

(iii) If $\mu$ is the right special word among the words $u$ and $v$, then $\mu^{2} \in \mathcal{L}(\alpha)$.

(iv) If $\lambda$ is the word among the words $u$ and $v$ which is not right special, then $\lambda^{2} \in \mathcal{L}(\alpha)$ if and only if $n=q_{1}$ or $l=a_{k}$.

Proof. Suppose first that $n=q_{1}$. Then it is straightforward to see that the factors $u$ and $v$ of length $n$ having intervals with endpoint $1-\alpha$ are $010^{a_{1}-2}=S_{2}$ and $10^{a_{1}-1}=S_{4}$. Clearly $S_{4}$ is right special and $L\left(S_{4}\right)=S_{2}$. Moreover $S_{2}^{2}, S_{4}^{2} \in \mathcal{L}(\alpha)$.

Assume that $n=q_{k, l}$ for some $k \geq 2$ with $0<l \leq a_{k}$. By Proposition 2.4 the point $\{-n \alpha\}$ is the point closest to 0 on the side opposite to the point $\left\{-q_{k-1} \alpha\right\}$. Thus either $\{-(n+1) \alpha\} \in[u]$ or $\{-(n+1) \alpha\} \in[v]$. Assume by symmetry that $\{-(n+1) \alpha\} \in[u]$. This means that the word $u$ is right special, proving (ii). Further, the endpoint of $[u]$ which is not $1-\alpha$ must be after a rotation the next closest point to 0 on the side opposite to the point $\left\{-q_{k-1} \alpha\right\}$. Thus by Proposition 2.4 $[u]=I\left(-\left(q_{k, l-1}+1\right) \alpha, 1-\alpha\right)$ and consequently $[v]=I\left(1-\alpha,-\left(q_{k-1}+1\right) \alpha\right)$.

Since the points $x=\left\{\left(-\left(q_{k, l-1}+1\right) \alpha\right\}\right.$ and $y=\left\{-\left(q_{k-1}+1\right) \alpha\right\}$ are on the opposite sides of the point $1-\alpha$ and the points $\{x+\alpha\}$ and $\{y+\alpha\}$ are on the opposite sides of the point 0 , it follows that $u$ begins with $c d$ and $v$ begins with $d c$ for distinct letters $c$ and $d$. Assume on the contrary that $u=c d z e u^{\prime}$ and $v=d c z f v^{\prime}$ for distinct letters $e$ and $f$. In particular, $|z| \leq n-3$. This means that the point $x^{\prime}=\{x+(|z|+2) \alpha\}$ is in $[e]$ and the point $y^{\prime}=\{y+(|z|+2) \alpha\}$ is in $[f]$. It must be that $e=c$ and $f=d$ as otherwise the point $x^{\prime}-\alpha$ would be in $[c]$ and the point $y^{\prime}-\alpha$ would be in $[d]$ contradicting the choice of $z$. Since $\alpha$ is irrational, either $x^{\prime}$ is closer to $1-\alpha$ than $x$ or $y^{\prime}$ is closer to $1-\alpha$ than $y$.

Suppose that $x^{\prime}$ is closer to $1-\alpha$ than $x$. Since $x^{\prime}$ is on the same side of the point $1-\alpha$ as $x$, it follows that

$$
\left\|x^{\prime}+\alpha\right\|=\left\|\left(q_{k, l-1}-|z|-2\right) \alpha\right\|<\left\|q_{k, l-1} \alpha\right\|=\|x+\alpha\| .
$$

Since $q_{k, l-1}-|z|-2<q_{k, l-1}$, by Proposition 2.4 it must be that $q_{k, l-1}-|z|-2 \leq 0$. However, as $\left\|q_{k, l-1} \alpha\right\|=\left\|-q_{k, l-1} \alpha\right\|$, it follows by Proposition 2.4 that $|z|+2-q_{k, l-1}=m q_{k-1}$ for some $m \geq 1$. Thus $|z|+2 \geq q_{k, l-1}+q_{k-1}=q_{k, l}=n$. This is, however, a contradiction as $|z| \leq n-3$.

Suppose then that $y^{\prime}$ is closer to $1-\alpha$ than $y$. Similar to above, it follows that

$$
\left\|y^{\prime}+\alpha\right\|=\left\|\left(q_{k-1}-|z|-2\right) \alpha\right\|<\left\|q_{k-1} \alpha\right\|=\|y+\alpha\| .
$$

Again, it must be that $q_{k-1}-|z|-2 \leq 0$. Since $\left\|q_{k-1} \alpha\right\|=\left\|-q_{k-1} \alpha\right\|$, it follows from (5) that $|z|+2-q_{k-1} \geq q_{k}$. Therefore $|z|+2 \geq q_{k}+q_{k-1}>n$. This is again a contradiction with the fact that $|z| \leq n-3$.

Thus we conclude that $u=c d w$ and $v=d c w$ for some word $w$ proving (i). As $n=q_{k, l}$, it must be that the right special word of length $n$ equals $\widetilde{s}_{k, l}$. Since $u$ and $v$ are conjugate by Proposition 2.6 (iii), Proposition 2.6 implies that if $l=a_{k}$, then $u^{2}, v^{2} \in \mathcal{L}(\alpha)$. Suppose that $l \neq a_{k}$. By Proposition 2.6, the word $s_{k, l}$ occurs as a square in $\mathcal{L}(\alpha)$. Since $\mathcal{L}(\alpha)$ is mirror-invariant, also $u^{2}=\widetilde{s}_{k, l}^{2} \in \mathcal{L}(\alpha)$. Therefore from Proposition 2.6 it follows that $|[u]|=\left\|q_{k, l-1} \alpha\right\|=\left|\left[s_{k, l}\right]\right|$. Now $[v]=I\left(1-\alpha,-\left(q_{k-1}+1\right) \alpha\right)$, so $|[v]|=\left\|q_{k-1} \alpha\right\| \neq|[u]|$. Thus Proposition 2.6 implies that $v^{2} \notin \mathcal{L}(\alpha)$. Hence (iii) and (iv) are proved.

Proof of Theorem 4.1. If $|w|=1$, then clearly $w=0=\widetilde{s}_{0}$, so the claim holds. We may thus focus on the case that $|w|>1$.

Suppose that $w^{2} \in \mathcal{L}(\alpha)$ satisfies the square root condition. By Corollary 4.3 the interval $[w]$ has $1-\alpha$ as an endpoint. Moreover, Proposition 2.5 implies that $|w|=q_{1}$ or $|w|=q_{k, l}$ for some $k \geq 2$ with $0<l \leq a_{k}$. Thus from Proposition 4.4 it follows that $w=\widetilde{s}$ or $w=L(\widetilde{s})$ where $s$ is the (semi)standard word of length $|w|$. By Proposition 4.4 we have that $\widetilde{s}^{2} \in \mathcal{L}(\alpha)$. Moreover, 
by Proposition 4.4 we have that $L(\widetilde{s})^{2} \in \mathcal{L}(\alpha)$ if and only if $|w|=q_{k}$ for some $k \geq 1$. Thus $w \in$ RStand $^{+}(\alpha) \cup L(R \operatorname{Rtand}(\alpha))$.

Suppose then that $w \in \operatorname{RStand}^{+}(\alpha) \cup L(\operatorname{RStand}(\alpha))$. Note first that $L(w)$ has the same number of letters 0 as $w$, so $w$ is conjugate to $L(w)$ by Proposition 2.6. Thus it follows from Proposition 2.6 that $w^{2} \in \mathcal{L}(\alpha)$. Let $u$ and $v$ be the factors of length $|w|$ having endpoint $1-\alpha$. By Proposition 4.4 the word $u$ must be right special and $v=L(u)$. Since the right special factor of length $|w|$ is unique, either $w=u$ or $L(w)=u$. Thus the interval $[w]$ has $1-\alpha$ as an endpoint. Then clearly $w^{2}$ satisfies the square root condition.

\section{Characterization by a Word Equation}

It turns out that the squares of slope $\alpha$ satisfying the square root condition have also a different characterization in terms of specific solutions of the word equation

$$
X_{1}^{2} X_{2}^{2} \cdots X_{n}^{2}=\left(X_{1} X_{2} \cdots X_{n}\right)^{2}
$$

in the language $\mathcal{L}(\alpha)$. We are interested only in the solutions of (8) where all words $X_{i}$ are minimal square roots (1), i.e., primitive roots of minimal squares. Thus we give the following definition.

Definition 5.1. A nonempty word $w$ is a solution to (8) if $w$ can be written as a product of minimal square roots $w=X_{1} X_{2} \cdots X_{n}$ which satisfy the word equation (8). The solution is trivial if $X_{1}=$ $X_{2}=\ldots=X_{n}$ and primitive if $w$ is primitive. The word $w$ is a solution to (8) in $\mathcal{L}(\alpha)$ if $w$ is a solution to (8) and $w^{2} \in \mathcal{L}(\alpha)$.

All minimal square roots of slope $\alpha$ are trivial solutions to (8). One example of a nontrivial solution is $w=S_{2} S_{1} S_{4}$ in the language of the Fibonacci word (i.e., in the language of slope $[0 ; 2,1,1, \ldots])$ since $w^{2}=(01010)^{2}=(01)^{2} \cdot 0^{2} \cdot(10)^{2}=S_{2}^{2} S_{1}^{2} S_{4}^{2}$. Note that in the language of any Sturmian word there are only finitely many trivial solutions as the index of every factor is finite.

Note that the factorization of a word as product of minimal squares is unique. Indeed, if $X_{1}^{2} \cdots X_{n}^{2}=Y_{1}^{2} \cdots Y_{m}^{2}$, where the squares $X_{i}^{2}$ and $Y_{i}^{2}$ are minimal, then either $X_{1}^{2}$ is a prefix of $Y_{1}^{2}$ or vice versa. Therefore by minimality $X_{1}^{2}=Y_{1}^{2}$, that is, $X_{1}=Y_{1}$. The uniqueness of the factorization follows.

Our aim is to complete the characterization of Theorem 4.1 as follows.

Theorem 5.2. Let $w \in \mathcal{L}(\alpha)$. The following are equivalent:

(i) $w$ is a primitive solution to (8) in $\mathcal{L}(\alpha)$,

(ii) $w^{2}$ satisfies the square root condition,

(iii) $w \in \operatorname{RStand}^{+}(\alpha) \cup L(R \operatorname{Rtand}(\alpha))$.

For later use in Section 8 we define the language $\mathcal{L}(\mathfrak{a}, \mathfrak{b})$.

Definition 5.3. The language $\mathcal{L}(\mathfrak{a}, \mathfrak{b})$ consists of all factors of the infinite words in the language

$$
\left(10^{\mathfrak{a}+1}\left(10^{\mathfrak{a}}\right)^{\mathfrak{b}}+10^{\mathfrak{a}+1}\left(10^{\mathfrak{a}}\right)^{\mathfrak{b}+1}\right)^{\omega}=\left(S_{5}+S_{6}\right)^{\omega} .
$$

Observe that by Proposition 2.2 every factor in $\mathcal{L}(\mathfrak{a}, \mathfrak{b})$ is a factor of some optimal squareful word with parameters $\mathfrak{a}$ and $\mathfrak{b}$. Moreover, if $\alpha=[0 ; \mathfrak{a}+1, \mathfrak{b}+1, \ldots]$, then $\mathcal{L}(\alpha) \subseteq \mathcal{L}(\mathfrak{a}, \mathfrak{b})$.

Definition 5.4. The language $\Pi(\mathfrak{a}, \mathfrak{b})$ consists of all nonempty words in $\mathcal{L}(\mathfrak{a}, \mathfrak{b})$ which can be written as products of the minimal squares (1). 
Let $w \in \Pi(\mathfrak{a}, \mathfrak{b})$, that is, $w=X_{1}^{2} \cdots X_{n}^{2}$ for minimal square roots $X_{i}$. Then we can define the square root of $w$ by setting $\sqrt{w}=X_{1} \cdots X_{n}$.

We need two technical lemmas. Their proofs are straightforward case-by-case analysis. The statement of Lemma 5.5 has a technical condition for later use in Section 8, which is perhaps better understood if the reader first reads the proof of Lemma 5.6 up to the point where Lemma 5.5 is invoked.

Lemma 5.5. Let $u$ and $v$ be words such that

- $u$ is a nonempty suffix of $S_{6}$,

- $|v| \geq\left|S_{5} S_{6}\right|$,

- $v$ begins with $x y$ for distinct letters $x$ and $y$,

- $u v \in \mathcal{L}(\mathfrak{a}, \mathfrak{b})$ and $L(v) \in \mathcal{L}(\mathfrak{a}, \mathfrak{b})$.

Suppose there exists a minimal square $X^{2}$ such that $\left|X^{2}\right|>|u|$ and $X^{2}$ is a prefix of $u v$ or $u L(v)$. Then there exist minimal squares $Y_{1}^{2}, \ldots, Y_{n}^{2}$ such that $X^{2}$ and $Y_{1}^{2} \ldots Y_{n}^{2}$ are prefixes of $u v$ and $u L(v)$ of the same length and $X=Y_{1} \cdots Y_{n}$.

Proof. Let $Z^{2}$ be a minimal square such that $\left|Z^{2}\right|>|u|$ and $Z^{2}$ is a prefix of $u v$ or $u L(v)$. It is not obvious at this point that $Z$ exists but its existence becomes evident as this proof progresses. By symmetry we may assume that $Z^{2}$ is a prefix of $u v$. To prove the claim we consider different cases depending on the word $Z$.

Case A. $Z=S_{1}=0$. Since $u$ is a nonempty suffix of $S_{6}$ and $\left|Z^{2}\right|>|u|$, it must be that $u=0$. As $v$ begins with 0 , we have that $v$ begins with 01 by assumption. Since $v \in \mathcal{L}(\mathfrak{a}, \mathfrak{b})$ and $|v| \geq\left|S_{6}\right|$, the word $v$ begins with either $010^{\mathfrak{a}} 10^{\mathfrak{a}}$ or $010^{\mathfrak{a}+1} 10^{\mathfrak{a}}$. In the latter case $L(v)$ would begin with $10^{\mathfrak{a}+2} 1$ contradicting the assumption $L(v) \in \mathcal{L}(\mathfrak{a}, \mathfrak{b})$. Hence $v$ begins with $010^{\mathfrak{a}} 10^{\mathfrak{a}}$. It follows that $u v$ has $0010^{\mathfrak{a}} 10^{\mathfrak{a}}$ as a prefix, that is, $u v$ begins with $S_{1}^{2} S_{4}^{2}$. On the other hand, the word $u L(v)$ has the word $S_{3}^{2}=010^{\mathfrak{a}+1} 10^{\mathfrak{a}}$ as a prefix. Since $S_{3}=S_{1} S_{4}$, the conclusion of the claim holds.

Case B. $Z=S_{2}=010^{\mathfrak{a}-1}$. If $u=0$, then $v$ has $10^{\mathfrak{a}} 10^{\mathfrak{a}}$ as a prefix and, consequently, $L(v)$ has $10^{\mathfrak{a}-1} 10^{\mathfrak{a}}$ as a prefix contradicting the fact that $L(v) \in \mathcal{L}(\mathfrak{a}, \mathfrak{b})$. Therefore by the assumptions that $u$ is a nonempty suffix of $S_{6}$ and $\left|Z^{2}\right|>|u|$, it follows that $u=010^{\mathfrak{a}}$. Thus $v$ has $10^{\mathfrak{a}}$ as a prefix. Using the fact that $L(v) \in \mathcal{L}(\mathfrak{a}, \mathfrak{b})$, we see that $v$ begins with $10^{\mathfrak{a}+1}$ and $L(v)$ begins with $010^{\mathfrak{a}}$. Hence $u v$ has $S_{2}^{2} S_{1}^{2}$ as a prefix, and $u L(v)$ has $S_{3}^{2}$ as a prefix. Since $S_{2} S_{1}=S_{3}$, we conclude, as in the previous case, that the conclusion holds.

Case C. $Z=S_{3}=010^{\mathfrak{a}}$. Using again the fact that $u$ is a suffix of $S_{6}$ and $\left|Z^{2}\right|>|u|$, we see that either $u=0$ or $u=010^{\mathfrak{a}}$. In the first case $v$ begins with $10^{\mathfrak{a}+1} 10^{\mathfrak{a}}$ and $L(v)$ begins with $010^{\mathfrak{a}} 10^{\mathfrak{a}}$. Hence the word $u L(v)$ has $S_{1}^{2} S_{4}^{2}$ as a prefix. As $S_{1} S_{4}=S_{3}$, the conclusion follows. Let us then consider the other case. Now $L(v)$ begins with $10^{\mathfrak{a}+1}$, so the word $u L(v)$ has $S_{2}^{2} S_{1}^{2}$ as a prefix. Again, the conclusion follows since $S_{2} S_{1}=S_{3}$.

Case D. $Z=S_{4}=10^{\mathfrak{a}}$. Now the only option is that $u=10^{\mathfrak{a}}$. Using the fact that $v \in \mathcal{L}(\mathfrak{a}, \mathfrak{b})$, we see that $v$ cannot begin with $10^{\mathfrak{a}} 1$, so $v$ must have $10^{\mathfrak{a}+1}$ as a prefix. Further, since $|v| \geq\left|S_{6}\right|$, it must be that $S_{6}$ is a prefix of $v$. If $S_{6} 1$ would be a prefix of $v$, then the word $L(v)$ would have the word $\left(10^{\mathfrak{a}}\right)^{\mathfrak{b}+2} 1$ as a factor contradicting the fact that $L(v) \in \mathcal{L}(\mathfrak{a}, \mathfrak{b})$. Thus $S_{6} 0$ is a prefix of $v$. Since $v \in \mathcal{L}(\mathfrak{a}, \mathfrak{b})$ and $|v| \geq\left|S_{5} S_{6}\right|$, we have that $S_{6} 0\left(10^{\mathfrak{a}}\right)^{\mathfrak{b}+1}=S_{5}^{2} 10^{\mathfrak{a}}$ is a prefix of $v$. Consequently, the word $L(v)$ begins with $0\left(10^{\mathfrak{a}}\right)^{\mathfrak{b}+1} 10^{\mathfrak{a}+1}\left(10^{\mathfrak{a}}\right)^{\mathfrak{b}+1}$, so $u L(v)$ has $S_{6}^{2}$ as a prefix. Assume first that $\mathfrak{b}$ is odd. It is straightforward to see that in this case

$$
0\left(10^{\mathfrak{a}}\right)^{\mathfrak{b}} 10^{\mathfrak{a}+1}\left(10^{\mathfrak{a}}\right)^{\mathfrak{b}+1}=\left(S_{2}^{2}\right)^{(\mathfrak{b}+1) / 2} S_{1}^{2}\left(S_{4}^{2}\right)^{(\mathfrak{b}+1) / 2} .
$$

Thus for the prefix $10^{\mathfrak{a}} S_{5} 10^{\mathfrak{a}}$ of $u v$ we have that

$$
10^{\mathfrak{a}} S_{5}^{2} 10^{\mathfrak{a}}=S_{4}^{2}\left(S_{2}^{2}\right)^{(\mathfrak{b}+1) / 2} S_{1}^{2}\left(S_{4}^{2}\right)^{(\mathfrak{b}+1) / 2} .
$$


As $S_{6}=S_{4} S_{2}^{(\mathfrak{b}+1) / 2} S_{1} S_{4}^{(\mathfrak{b}+1) / 2}$, the conclusion follows as before. Assume then that $\mathfrak{b}$ is even. It is now easy to show that

$$
0\left(10^{\mathfrak{a}}\right)^{\mathfrak{b}} 10^{\mathfrak{a}+1}\left(10^{\mathfrak{a}}\right)^{\mathfrak{b}+1}=\left(S_{2}^{2}\right)^{\mathfrak{b} / 2} S_{3}^{2}\left(S_{4}^{2}\right)^{\mathfrak{b} / 2} .
$$

Therefore

$$
10^{\mathfrak{a}} S_{5}^{2} 10^{\mathfrak{a}}=S_{4}^{2}\left(S_{2}^{2}\right)^{\mathfrak{b} / 2} S_{3}^{2}\left(S_{4}^{2}\right)^{\mathfrak{b} / 2} .
$$

Since $S_{6}=S_{4} S_{2}^{\mathfrak{b} / 2} S_{3} S_{4}^{\mathfrak{b} / 2}$, the conclusion again follows.

Case E. $Z=S_{5}=10^{\mathfrak{a}+1}\left(10^{\mathfrak{a}}\right)^{\mathfrak{b}}$. Now either $u=10^{\mathfrak{a}}$ or $u=10^{\mathfrak{a}+1}\left(10^{\mathfrak{a}}\right)^{\mathfrak{b}+1}$. In the first case $v$ must begin with $0\left(10^{\mathfrak{a}}\right)^{\mathfrak{b}} 10^{\mathfrak{a}+1}\left(10^{\mathfrak{a}}\right)^{\mathfrak{b}}$. However, this implies that $L(v)$ begins with $10^{\mathfrak{a}+1}\left(10^{\mathfrak{a}}\right)^{\mathfrak{b}-1} 10^{\mathfrak{a}+1}\left(10^{\mathfrak{a}}\right)^{\mathfrak{b}}$ contradicting the fact that $L(v) \in \mathcal{L}(\mathfrak{a}, \mathfrak{b})$. Consider then the latter case where $v$ begins with $0\left(10^{\mathfrak{a}}\right)^{\mathfrak{b}}$. As $L(v) \in \mathcal{L}(\mathfrak{a}, \mathfrak{b})$ and $|v| \geq\left|S_{6}\right|$, it must be that $L(v)$ begins with $10^{\mathfrak{a}+1}\left(10^{\mathfrak{a}}\right)^{\mathfrak{b}+1}$. Hence the word $u L(v)$ has $S_{6}^{2}$ as a prefix. Since the word $v$ begins with $0\left(10^{\mathfrak{a}}\right)^{\mathfrak{b}+2}$, the word $u v$ has $S_{5}^{2} S_{4}^{2}$ as a prefix. The conclusion follows as $S_{5} S_{4}=S_{6}$.

Case F. $Z=S_{6}=10^{\mathfrak{a}+1}\left(10^{\mathfrak{a}}\right)^{\mathfrak{b}+1}$. Now there are two possibilities: either $u=10^{\mathfrak{a}}$ or $u=10^{\mathfrak{a}+1}\left(10^{\mathfrak{a}}\right)^{\mathfrak{b}+1}$. In the first case $v$ begins with $0\left(10^{\mathfrak{a}}\right)^{\mathfrak{b}+1} 10^{\mathfrak{a}+1}\left(10^{\mathfrak{a}}\right)^{\mathfrak{b}+1}$, so $L(v)$ begins with $10^{\mathfrak{a}+1}\left(10^{\mathfrak{a}}\right)^{\mathfrak{b}} 10^{\mathfrak{a}+1}\left(10^{\mathfrak{a}}\right)^{\mathfrak{b}+1}$. The word $u L(v)$ has $S_{4}^{2} 0\left(10^{\mathfrak{a}}\right)^{\mathfrak{b}} 10^{\mathfrak{a}+1}\left(10^{\mathfrak{a}}\right)^{\mathfrak{b}+1}$ as a prefix. Proceeding as in the Case D depending on the parity of $\mathfrak{b}$, we see that the conclusion holds. Consider then the latter case $u=10^{\mathfrak{a}+1}\left(10^{\mathfrak{a}}\right)^{\mathfrak{b}+1}$. The word $v$ must begin with $u$, so $L(v)$ has $0\left(10^{\mathfrak{a}}\right)^{\mathfrak{b}+2}$ as a prefix. Clearly the word $u L(v)$ has $S_{5}^{2} S_{4}^{2}$ as a prefix. As $S_{6}=S_{5} S_{4}$, the conclusion follows.

A more intuitive way of stating Lemma 5.5 is that under the assumptions of the lemma swapping two adjacent and distinct letters which do not occur as a prefix of a minimal square affects a product of minimal square only locally and does not change its square root.

Lemma 5.6. Let $w$ be a primitive solution to (8) having the word $S_{6}=10^{\mathfrak{a}+1}\left(10^{\mathfrak{a}}\right)^{\mathfrak{b}+1}$ as a suffix such that $w^{2}, L(w) \in \mathcal{L}(\mathfrak{a}, \mathfrak{b})$. Then $w L(w) \in \Pi(\mathfrak{a}, \mathfrak{b})$ and $\sqrt{w L(w)}=w$.

Proof. If $w=S_{6}$, then it is easy to see that $w L(w)=S_{5}^{2} S_{4}^{2}$ and $w=S_{5} S_{4}$, so the claim holds. We may thus suppose that $S_{6}$ is a proper suffix of $w$.

Since $w$ is a solution to (8), we have that $w^{2}=X_{1}^{2} \cdots X_{n}^{2}$ and $w=X_{1} \cdots X_{n}$ for some minimal square roots $X_{i}$. It must be that $n>1$ as if $n=1$ then $w=X_{1}$, and it is not possible for $S_{6}$ to be a proper suffix of $w$. Assume for a contradiction that $X_{1}=S_{1}$. Since $X_{1} X_{2}$ is a prefix of $w^{2}$, it follows that $X_{2}$ begins with the letter 0 . If $X_{2} \neq S_{1}$, then $X_{1} X_{2}$ begins with 001 but $X_{1}^{2} X_{2}^{2}$ begins with 000, which is impossible. Hence $X_{2}=S_{1}$, and by repeating the argument it follows that $X_{k}=S_{1}$ for all $k$ such that $1 \leq k \leq n$. Thus $w$ cannot have $S_{6}$ as a suffix, so we conclude that $X_{1} \neq S_{1}$. Hence $w$ always begins with 01 or 10 .

We show that $\left|X_{1}^{2}\right|<|w|$. Assume on the contrary that $\left|X_{1}^{2}\right| \geq|w|$. Since $w$ has the word $S_{6}$ as a suffix, it follows that $S_{6}$ is a factor of $X_{1}^{2}$. It follows that $X_{1}$ is one of the words $S_{5}, S_{6}$ or $S_{3}($ if $\mathfrak{b}=0)$. If $X_{1}=S_{5}$, then $S_{6}$ occurs in $X_{1}^{2}=10^{a+1}\left(10^{a}\right)^{b} 10^{a+1}\left(10^{a}\right)^{b}$ only as a prefix. Thus $w=S_{6}$ contradicting the fact that $S_{6}$ is a proper suffix of $w$. If $X_{1}=S_{6}$, then $S_{6}$ occurs in $X_{1}^{2}=10^{\mathfrak{a}+1}\left(10^{\mathfrak{a}}\right)^{\mathfrak{b}+1} 10^{\mathfrak{a}+1}\left(10^{\mathfrak{a}}\right)^{\mathfrak{b}+1}$ as a prefix and as a suffix. Since $w \neq S_{6}$, it must be that $w=X_{1}^{2}$ contradicting the primitivity of $w$. Let finally $\mathfrak{b}=0$ and $X_{1}=S_{3}$. Then $S_{6}$ occurs in $X_{1}^{2}=010^{\mathfrak{a}+1} 10^{\mathfrak{a}}$ as a suffix. Hence $w=X_{1}^{2}$ contradicting again the primitivity of $w$.

Now there exists a maximal $r$ such that $1 \leq r<n$ and $X_{1}^{2} \cdots X_{r}^{2}$ is a prefix of $w$. Actually $X_{1}^{2} \cdots X_{r}^{2}$ is a proper prefix of $w$, as otherwise $w^{2}=\left(X_{1}^{2} \cdots X_{r}^{2}\right)^{2}=\left(X_{1} \cdots X_{r} X_{1} \cdots X_{r}\right)^{2}$, so $w=\left(X_{1} \cdots X_{r}\right)^{2}$ contradicting the primitivity of $w$. Thus when factorizing $w L(w)$ and $w^{2}$ as products of minimal squares, the first $r$ squares are equal. Let $u$ be the nonempty word such that $w=X_{1}^{2} \cdots X_{r}^{2} u$. By the definition of the number $r$, we have that $u$ is a proper prefix of $X_{r+1}^{2}$. Suppose for a contradiction that $|u|>\left|S_{6}\right|$. It follows that $u$ has $S_{6}$ as a proper suffix. This leaves only the possibilities that $X_{r+1}$ is either of the words $S_{5}$ or $S_{6}$. However, if $X_{r+1}=S_{5}$, 
then $S_{6}$ cannot be a proper suffix of $u$, and if $X_{r+1}=S_{6}$, then $r$ is not maximal. We conclude that $|u| \leq\left|S_{6}\right|$.

Next we show that $w$ must satisfy $|w| \geq\left|S_{5} S_{6}\right|$. Suppose first that $w$ begins with the letter 0 . Then as $S_{6}$ is a proper suffix of $w$ and $w^{2} \in \mathcal{L}(\mathfrak{a}, \mathfrak{b})$, it must be that $w$ begins with $0\left(10^{\mathfrak{a}}\right)^{\mathfrak{b}+1}$. Suppose that this prefix overlaps with the suffix $S_{6}$. Then clearly $w=0\left(10^{\mathfrak{a}}\right)^{\mathfrak{b}} 10^{\mathfrak{a}+1}\left(10^{\mathfrak{a}}\right)^{\mathfrak{b}+1}=$ $\left(0\left(10^{\mathfrak{a}}\right)^{\mathfrak{b}+1}\right)^{2}$ contradicting the primitivity of $w$. If the prefix $0\left(10^{\mathfrak{a}}\right)^{\mathfrak{b}+1}$ does not overlap with the suffix $S_{6}$, then $|w| \geq\left|S_{5} S_{6}\right|$. Assume then that $w$ begins with the letter 1 . Similar to above, the word $w$ must begin with $10^{\mathfrak{a}+1}\left(10^{\mathfrak{a}}\right)^{\mathfrak{b}+1}$. In this case necessarily $|w| \geq\left|S_{5} S_{6}\right|$.

Finally, we can apply Lemma 5.5 to the words $u$ and $w$ with $X=X_{r+1}$. We obtain minimal squares $Y_{1}^{2}, \ldots, Y_{m}^{2}$ such that $Y_{1}^{2} \cdots Y_{m}^{2}$ is a prefix of $u L(w)$ and and $Y_{1} \cdots Y_{m}=X_{r+1} \cdots X_{r+t}$ for some $t \geq 1$. Thus

$$
\begin{aligned}
w L(w) & =X_{1}^{2} \cdots X_{r}^{2} Y_{1}^{2} \cdots Y_{m}^{2} X_{r+t+1}^{2} \cdots X_{n}^{2} \quad \text { and } \\
w & =X_{1} \cdots X_{n}=X_{1} \cdots X_{r} Y_{1} \cdots Y_{m} X_{r+t+1} \cdots X_{n} .
\end{aligned}
$$

The claim is proved.

Proposition 5.7. Let $w \in R S \operatorname{tand}{ }^{+}(\alpha) \cup L(R \operatorname{Rtand}(\alpha))$. Then the word $w$ is a primitive solution to (8) in $\mathcal{L}(\alpha)$.

Proof. Note that Proposition 2.6 implies that $w^{2} \in \mathcal{L}(\alpha)$. Suppose first that $|w|<\left|S_{6}\right|$ where $S_{6}=\widetilde{s}_{3,1}=10^{\mathfrak{a}+1}\left(10^{\mathfrak{a}}\right)^{\mathfrak{b}+1}$. Clearly the minimal square root $S_{1}, \ldots, S_{5}$ are solutions to (8), so we are left with the case where $w=\widetilde{s}_{2, \ell}=0\left(10^{\mathfrak{a}}\right)^{\ell}$ for some $\ell$ such that $1<\ell \leq \mathfrak{b}+1$. It is straightforward to see that if $\ell$ is even, then

$$
w^{2}=\left(S_{2}^{2}\right)^{\ell / 2} S_{1}^{2}\left(S_{4}^{2}\right)^{\ell / 2} \text { and } w=S_{2}^{\ell / 2} S_{1} S_{4}^{\ell / 2} .
$$

If $\ell$ is odd, then

$$
w^{2}=\left(S_{2}^{2}\right)^{(\ell+1) / 2} S_{3}^{2}\left(S_{4}^{2}\right)^{(\ell+1) / 2} \text { and } w=S_{2}^{(\ell+1) / 2} S_{3} S_{4}^{(\ell+1) / 2} .
$$

Hence $w$ is a solution to (8).

We may thus suppose that $|w| \geq\left|S_{6}\right|$, so $w$ has $S_{6}$ as a suffix. We proceed by induction. Now either $w=\widetilde{s}_{k, \ell}$ for some $k \geq 3$ with $0<\ell \leq a_{k}$ or $L(w)=\widetilde{s}_{k}$ for some $k \geq 3$. We assume that the claim holds for every word satisfying the hypotheses which are shorter than $w$. Consider first the case $w=\widetilde{s}_{k, \ell}$ for some $k \geq 3$ with $0<\ell \leq a_{k}$. By the fact that $\widetilde{s}_{k-1} \widetilde{s}_{k-2}=L\left(\widetilde{s}_{k-2}\right) \widetilde{s}_{k-1}$ we obtain that

$$
w^{2}=\widetilde{s}_{k-2} \widetilde{s}_{k-1}^{\ell} \widetilde{s}_{k-2} \widetilde{s}_{k-1}^{\ell}=\widetilde{s}_{k-2} \widetilde{s}_{k-1}^{\ell-1} L\left(\widetilde{s}_{k-2}\right) \widetilde{s}_{k-1}^{\ell-1} \cdot \widetilde{s}_{k-1}^{2}=\widetilde{s}_{k, \ell-1} L\left(\widetilde{s}_{k, \ell-1}\right) \cdot \widetilde{s}_{k-1}^{2} .
$$

Now if $k=3$ and $\ell=1$, then the conclusion holds as $\widetilde{s}_{3,1}=S_{6}$ is a minimal square root. Hence we may assume that either $k>3$ or $k=3$ and $\ell>1$. Since $\widetilde{s}_{k-1}$ is a solution to (8), we have that $\widetilde{s}_{k-1}^{2}=X_{1}^{2} \cdots X_{n}^{2}$ and $\widetilde{s}_{k-1}=X_{1} \cdots X_{n}$ for some minimal square roots $X_{i}$. In other words,

$$
\widetilde{s}_{k-1}^{2} \in \Pi(\mathfrak{a}, \mathfrak{b}) \text { and } \sqrt{\widetilde{s}_{k-1}^{2}}=\widetilde{s}_{k-1} \text {. }
$$

Since $\left|\widetilde{s}_{k, \ell-1}\right| \geq\left|S_{6}\right|$, with an application of Lemma 5.6 we obtain that

$$
\widetilde{s}_{k, \ell-1} L\left(\widetilde{s}_{k, \ell-1}\right) \in \Pi(\mathfrak{a}, \mathfrak{b}) \text { and } \sqrt{\widetilde{s}_{k, \ell-1} L\left(\widetilde{s}_{k, \ell-1}\right)}=\widetilde{s}_{k, \ell-1} .
$$

Thus $w^{2} \in \Pi(\mathfrak{a}, \mathfrak{b})$ and

$$
\sqrt{w^{2}}=\sqrt{\widetilde{s}_{k, \ell-1} L\left(\widetilde{s}_{k, \ell-1}\right)} \sqrt{\widetilde{s}_{k-1}^{2}}=\widetilde{s}_{k, \ell-1} \widetilde{s}_{k-1}=w,
$$


so $w$ is a solution to (8).

Consider next the case $w=L\left(\widetilde{s}_{k}\right)$ for some $k \geq 3$. Similar to above,

$$
\begin{aligned}
w^{2} & =L\left(\widetilde{s}_{k-2}\right) \widetilde{s}_{k-1}^{a_{k}} L\left(\widetilde{s}_{k-2}\right) \widetilde{s}_{k-1}^{a_{k}}=L\left(\widetilde{s}_{k-2}\right) \widetilde{s}_{k-1}^{a_{k}+1} \widetilde{s}_{k-2} \widetilde{s}_{k-1}^{a_{k}-1} \\
& =L\left(\widetilde{s}_{k-2}\right) \widetilde{s}_{k-1} \widetilde{s}_{k-3} \widetilde{s}_{k-2}^{a_{k-1}} \widetilde{s}_{k-1}^{a_{k}-1} \widetilde{s}_{k-2} \widetilde{s}_{k-1}^{a_{k}-1}=L\left(\widetilde{s}_{k-2}\right) \widetilde{s}_{k-1} \widetilde{s}_{k-3} \widetilde{s}_{k-2}^{a_{k-1}-1} \cdot \widetilde{s}_{k, a_{k}-1}^{2} \\
& =\widetilde{s}_{k-1} \widetilde{s}_{k-2} \widetilde{s}_{k-3} \widetilde{s}_{k-2}^{a_{k-1}-1} \cdot \widetilde{s}_{k, a_{k}-1}^{2}=\widetilde{s}_{k-1} L\left(\widetilde{s}_{k-1}\right) \cdot \widetilde{s}_{k, a_{k}-1}^{2} .
\end{aligned}
$$

If $k>3$, then the claim follows using the induction hypothesis and Lemma 5.6 as above. In the case $k=3$ we have that

$$
\widetilde{s}_{k-1} L\left(\widetilde{s}_{k-1}\right) \in \Pi(\mathfrak{a}, \mathfrak{b}) \text { and } \sqrt{\widetilde{s}_{k-1} L\left(\widetilde{s}_{k-1}\right)}=\widetilde{s}_{k-1} .
$$

Namely, it is not difficult to see that if $\mathfrak{b}$ is even, then

$$
\widetilde{s}_{k-1} L\left(\widetilde{s}_{k-1}\right)=\left(S_{2}^{2}\right)^{1+\mathfrak{b} / 2} S_{1}^{2}\left(S_{4}^{2}\right)^{\mathfrak{b} / 2} \text { and } \widetilde{s}_{k-1}=S_{2}^{1+\mathfrak{b} / 2} S_{1} S_{4}^{\mathfrak{b} / 2} .
$$

If $\mathfrak{b}$ is odd, then

$$
\widetilde{s}_{k-1} L\left(\widetilde{s}_{k-1}\right)=\left(S_{2}^{2}\right)^{(\mathfrak{b}+1) / 2} S_{3}^{2}\left(S_{4}^{2}\right)^{(\mathfrak{b}-1) / 2} \text { and } \widetilde{s}_{k-1}=S_{2}^{(\mathfrak{b}+1) / 2} S_{3} S_{4}^{(\mathfrak{b}-1) / 2} .
$$

Thus $w$ is a solution to (8) also in the case $k=3$.

Note that a word $w$ in the set $L\left(R S \operatorname{Rtand} d^{+}(\alpha)\right) \backslash L(R \operatorname{Rtand}(\alpha))$ is a solution to (8) but not in the language $\mathcal{L}(\alpha)$. Rather, $w$ is a solution to $(8)$ in $\mathcal{L}(\beta)$ where $\beta$ is a suitable irrational such that $L(w)$ is a reversed standard word of slope $\beta$.

From Proposition 5.7 we conclude the following interesting fact:

Corollary 5.8. There exist arbitrarily long primitive solutions of (8) in $\mathcal{L}(\alpha)$.

We can now prove Theorem 5.2.

Proof of Theorem 5.2. By Proposition 5.7 and Theorem 4.1 it is sufficient to prove that (i) implies (ii).

Suppose that $w$ is a solution to (8) in $\mathcal{L}(\alpha)$. Write $w^{2}$ as a product of minimal squares: $w^{2}=$ $X_{1}^{2} X_{2}^{2} \cdots X_{n}^{2}$. Let $x \in\left[w^{2}\right]$. Then the word $s_{x, \alpha}$ begins with $X_{1}^{2} X_{2}^{2} \cdots X_{n}^{2}$, so by Theorem 3.2 the word $\sqrt{s_{x, \alpha}}=s_{\psi(x), \alpha}$ begins with $X_{1} X_{2} \cdots X_{n}$. Therefore $\psi(x) \in\left[X_{1} X_{2} \cdots X_{n}\right]=[w]$. Thus $w^{2}$ satisfies the square root condition.

\section{A More Detailed Combinatorial Description of the Square Root Map}

Recall from Section 3 that the square root $\sqrt{s}$ of a Sturmian word $s$ has the same factors as $s$. The proofs were dynamical; we used the special mapping $\psi$ on the circle. In this section we describe combinatorially why the language is preserved; we give a location for any prefix of $\sqrt{s}$ in $s$. As a side product, we are able to describe when a Sturmian word is uniquely factorizable as a product of squares of reversed (semi)standard words.

Let us begin with an introductory example. Recall from Section 3 the square root of the Fibonacci word $f$ :

$$
\begin{aligned}
f & =(010)^{2}(100)^{2}(10)^{2}(01)^{2} 0^{2}(10010)^{2}(01)^{2} \cdots, \\
\sqrt{f} & =010 \cdot 100 \cdot 10 \cdot 01 \cdot 0 \cdot 10010 \cdot 01 \cdots .
\end{aligned}
$$

Obviously the square root $X_{1}=010$ of $(010)^{2}$ occurs as a prefix of $f$. Equally clearly the word $010 \cdot 100=\sqrt{(010)^{2}(100)^{2}}$ occurs, not as a prefix, but after the prefix $X_{1}$ of $f$. Thus the position of 
the first occurrence of $010 \cdot 100$ shifted $\left|X_{1}\right|=3$ positions from the position of the first occurrence of $X_{1}$. However, when comparing the position of the first occurrence of $\sqrt{(010)^{2}(100)^{2}(10)^{2}}$ with the first occurrence of $010 \cdot 100$, we see that there is no further shift. By further inspection, the word $\sqrt{(010)^{2}(100)^{2}(10)^{2}(01)^{2} 0^{2}(10010)^{2}}$ occurs for the first time at position $\left|X_{1}\right|$ of $f$. This is no longer true for the first seven minimal squares; the first occurrence of $X_{1} X_{2}=010 \cdot 100 \cdot 10$. $01 \cdot 0 \cdot 10010 \cdot 01$ is at position $\left|X_{1} X_{2}\right|=16$ of $f$. The amount of shift from the previous position $\left|X_{1}\right|=3$ is $\left|X_{2}\right|=13$; observe that both of these numbers are Fibonacci numbers. Thus the amount of shift was exactly the length of the square roots added after observing the previous shift. As an observant reader might have noticed, both of the words $X_{1}$ and $X_{2}$ are reversed standard words, or equivalently, primitive solutions to (8). Repeating similar inspections on other Sturmian words suggests that there is a certain pattern to these shifts and that knowing the pattern would make it possible to locate prefixes of $\sqrt{s}$ in the Sturmian word $s$. Thus it makes very much sense to "accelerate" the square root map by considering squares of solutions to (8) instead of just minimal squares. Next we make these somewhat vague observations more precise.

Every Sturmian word has a solution of (8) as a square prefix. Next we aim to characterize Sturmian words having infinitely many solutions of (8) as square prefixes. The next two lemmas are key results towards such a characterization.

Lemma 6.1. Consider the reversed (semi)standard word $\widetilde{s}_{k, l}$ of slope $\alpha$ with $k \geq 2$ and $0<\ell \leq a_{k}$. The set $\left[\widetilde{s}_{k, \ell}\right] \backslash\{1-\alpha\}$ equals the disjoint union

$$
\left(\bigcup_{i=0}^{\infty} \bigcup_{j=1}^{a_{k+2 i}}\left[\widetilde{s}_{k+2 i, j}^{2}\right]\right) \backslash \bigcup_{i=1}^{l-1}\left[\widetilde{s}_{k, i}^{2}\right] .
$$

Analogous representations exist for the sets $\left[\widetilde{s}_{0}\right] \backslash\{1-\alpha\}$ and $\left[\widetilde{s}_{1}\right] \backslash\{1-\alpha\}$.

To put it more simply: for each $x \neq 1-\alpha$ there exists a unique reversed (semi)standard word $w$ such that $x \in\left[w^{2}\right]$. To illustrate the proof, we begin by giving a proof sketch.

Proof Sketch. Consider as an example the interval $[0]=I(0,1-\alpha)$. It is easy to see that $\left[0^{2}\right]=$ $I(0,-2 \alpha)=I\left(0,-\left(q_{0}+1\right) \alpha\right)$, so $[0]=\left[0^{2}\right] \cup I\left(-\left(q_{0}+1\right) \alpha, 1-\alpha\right)$. The interval $I\left(-\left(q_{0}+1\right) \alpha, 1-\right.$ $\alpha)$ is the interval of the factor $\widetilde{s}_{2,1}$. Therefore $[0]=\left[\widetilde{s}_{0}^{2}\right] \cup\left[\widetilde{s}_{2,1}\right]$. Since $\widetilde{s}_{2,1}^{2} \in \mathcal{L}(\alpha)$, the interval $\left[\widetilde{s}_{2,1}^{2}\right]$ splits into two parts: $\left[\widetilde{s}_{2,1}\right]=\left[\widetilde{s}_{2,1}^{2}\right] \cup J$. It is straightforward to show that $J=I\left(-\left(q_{2,1}+1\right) \alpha, 1-\alpha\right)$. Again, the interval $J$ is the interval of the factor $w$ which equals either $\widetilde{s}_{2,2}$ or $\widetilde{s}_{4,1}$ depending on the number $a_{2}$. So $[0]=\left[\widetilde{s}_{0}^{2}\right] \cup\left[\widetilde{s}_{2,1}^{2}\right] \cup[w]$. This process can be repeated for the interval $[w]$ and indefinitely after that. The very same idea can be applied to any interval $\left[\widetilde{s}_{k, \ell}\right]$.

Proof of Lemma 6.1. Consider the lengths of the reversed (semi)standard words beginning with the same letter as $\widetilde{s}_{k, \ell}$. Out of these lengths we can form the unique increasing sequence $\left(b_{n}\right)$ such that $b_{1}=q_{k, \ell-1}$. If we set $s_{1}=\widetilde{s}_{k, \ell}$ and $J_{1}=I\left(-\left(b_{1}+1\right) \alpha, 1-\alpha\right)$, then based on the observations in the proof of Proposition 4.4 we see that $J_{1}=\left[s_{1}\right]$. The interval $J_{1}$ is split by the point $\left\{-\left(q_{k, \ell}+1\right) \alpha\right\}=\left\{-\left(b_{2}+1\right) \alpha\right\}$. It must be that $\left[s_{1}^{2}\right]=I\left(-\left(b_{1}+1\right) \alpha,-\left(b_{2}+1\right) \alpha\right)$. Otherwise $\left[s_{1}^{2}\right]=\left[s_{1}\right] \cap R^{-b_{2}}\left(\left[s_{1}\right]\right)=I\left(-\left(b_{2}+1\right) \alpha, 1-\alpha\right)$, so the points $\left\{-\left(b_{1}+b_{2}\right) \alpha\right\}$ and $\left\{-b_{1} \alpha\right\}$ are on the opposite sides of 0 . Furthermore, $\left\|\left(b_{1}+b_{2}\right) \alpha\right\|$ equals the distance between the points $\left\{-b_{1} \alpha\right\}$ and $\left\{-b_{2} \alpha\right\}$, so $\left\|\left(b_{1}+b_{2}\right) \alpha\right\|=\left\|q_{k-1} \alpha\right\|$. Since also the point $\left\{-q_{k-1} \alpha\right\}$ is on the side opposite to $\left\{-b_{1} \alpha\right\}$, it follows that $q_{k-1}=b_{1}+b_{2}$ which is obviously false. Thus $J_{2}=J_{1} \backslash\left[s_{1}^{2}\right]=I\left(-\left(b_{2}+\right.\right.$ 1) $\alpha, 1-\alpha)$ is the interval of $s_{2}$, the unique reversed (semi)standard word of length $b_{3}$ beginning with the same letter as $s_{1}$. By repeating this when $n>1$, we see that the interval $J_{n}$ is split by the point $\left\{-\left(b_{n+1}+1\right) \alpha\right\}$ and that $\left[s_{n}^{2}\right]=I\left(-\left(b_{n}+1\right) \alpha,-\left(b_{n+1}+1\right) \alpha\right)$. Then there is a unique reversed (semi)standard word $s_{n+1}$ such that $\left[s_{n+1}\right]=I\left(-\left(b_{n+1}+1\right) \alpha, 1-\alpha\right)=J_{n} \backslash\left[s_{n}^{2}\right]$; we set $J_{n+1}=\left[s_{n+1}\right]$. By the definition of the sequence $\left(b_{n}\right)$, the words $s_{n+1}$ and $s_{1}$ begin with the same 
letter. This yields a well-defined sequence $\left(J_{n}\right)$ of nested subintervals of $J_{1}$. It is clear that $\left|J_{n}\right| \rightarrow 0$ as $n \rightarrow \infty$. It follows that

$$
\left[\widetilde{s}_{k, \ell}\right] \cup\{1-\alpha\}=J_{1} \cup\{1-\alpha\}=\bigcup_{n=1}^{\infty}\left[s_{n}^{2}\right] \cup\{1-\alpha\} .
$$

The sets $\left[s_{n}^{2}\right]$ are by definition disjoint. The claim follows since the indexing in the claim is just another way to express the reversed (semi)standard words having lengths from the sequence $\left(b_{n}\right)$.

The above proof works as it is for the cases $\widetilde{s}_{0}$ and $\widetilde{s}_{1}$; only minor adjustments in notation are needed.

Lemma 6.2. Let $u \in \operatorname{RStand}^{+}(\alpha)$ and $v \in \operatorname{RStand}^{+}(\alpha) \cup L\left(\operatorname{RStand}^{+}(\alpha)\right)$. Then $u^{2}$ is never a proper prefix of $v^{2}$.

Proof. If $v \in \operatorname{RStand}^{+}(\alpha)$ and $|u| \neq|v|$, then by Lemma 6.1, the intervals $\left[u^{2}\right]$ and $\left[v^{2}\right]$ are disjoint. Hence $u^{2}$ can never be a proper prefix of $v^{2}$. Assume then that $v \in L\left(\operatorname{RStand}^{+}(\alpha)\right)$. If $|v| \leq\left|\widetilde{s}_{1}\right|$, then $v^{2}$ is a minimal square, so it is not possible for $u^{2}$ to be a proper prefix of $v^{2}$. Suppose that $|v|=\left|\widetilde{s}_{k, \ell}\right|$ for some $k \geq 2$ with $0<\ell \leq a_{k}$. As in the proof of Proposition 4.4, we have that $[v]=I\left(-\left(q_{k-1}+1\right) \alpha, 1-\alpha\right)$. If $u$ begins with the same letter as $v$ and $|u|<|v|$, then $|u| \leq\left|\widetilde{s}_{k-1}\right|$. It follows, as in the proof of Lemma 6.1, that the distance between $1-\alpha$ and either of the endpoints of the interval $\left[u^{2}\right]$ must be at least $\left\|q_{k-1} \alpha\right\|$. Hence the intervals $[v]$ and $\left[u^{2}\right]$ are disjoint, so $u^{2}$ is not a proper prefix of $v^{2}$.

Let $s$ be a fixed Sturmian word of slope $\alpha$. Since the index of a factor of a Sturmian word is finite, Lemma 6.2 and Theorem 5.2 imply that if $s$ has infinitely many solutions of (8) as square prefixes then no word in $\operatorname{RStand}^{+}(\alpha)$ is a square prefix of $s$. We have now the proper tools to prove the following:

Proposition 6.3. Let $s_{x, \alpha}$ be a Sturmian word of slope $\alpha$ and intercept $x$. Then $s_{x, \alpha}$ begins with a square of a word in $\operatorname{RStand}^{+}(\alpha)$ if and only if $x \neq 1-\alpha$.

Proof. If $x \neq 1-\alpha$, then $x \in I_{0} \backslash\{1-\alpha\}=\left[\widetilde{s}_{0}\right] \backslash\{1-\alpha\}$ or $x \in I_{1} \backslash\{1-\alpha\}=\left[\widetilde{s}_{1}\right] \backslash\{1-\alpha\}$. Thus by applying Lemma 6.1 to $I_{0} \backslash\{1-\alpha\}$ or $I_{1} \backslash\{1-\alpha\}$, we see that the word $s_{x, \alpha}$ begins with a square of a word in $\operatorname{RStand}^{+}(\alpha)$.

Suppose then that $x=1-\alpha$. Then $s_{x, \alpha} \in\left\{01 c_{\alpha}, 10 c_{\alpha}\right\}$. It is a well-known fact that $s_{2 k}=$ $P_{2 k} 10$ and $s_{2 k+1}=Q_{2 k+1} 01$ for some palindromes $P_{2 k}$ and $Q_{2 k+1}$ for every $k \geq 1$ (see e.g. [11, Lemma 2.2.8]). As $c_{\alpha}=\lim _{k \rightarrow \infty} s_{k}$, it follows that $01 c_{\alpha}=\lim _{k \rightarrow \infty} \widetilde{s}_{2 k}$ and $10 c_{\alpha}=\lim _{k \rightarrow \infty} \widetilde{s}_{2 k+1}$. Hence by Lemma 6.2, the word $s_{x, \alpha}$ cannot have as a prefix a square of a word in $\operatorname{RStand}^{+}(\alpha)$.

It follows that if $s$ has infinitely many solutions of (8) as square prefixes, then $s \in\left\{01 c_{\alpha}, 10 c_{\alpha}\right\}$.

Next we take one extra step and characterize when $s$ can be written as a product of squares of words in $\operatorname{RStand}^{+}(\alpha)$.

Theorem 6.4. A Sturmian word $s$ of slope $\alpha$ can be written as a product of squares of words in $\operatorname{RStand}^{+}(\alpha)$ if and only if $s$ is not of the form $X_{1}^{2} X_{2}^{2} \cdots X_{n}^{2} c$ where $X_{i} \in \operatorname{RStand}^{+}(\alpha)$ and $c \in\left\{01 c_{\alpha}, 10 c_{\alpha}\right\}$. If $s$ is a product of squares in $\operatorname{RStand}^{+}(\alpha)$, then this product is unique.

Proof. This is a direct consequence of Proposition 6.3 and Lemma 6.2.

Suppose that $s \notin\left\{01 c_{\alpha}, 10 c_{\alpha}\right\}$. Then the word $s$ has only finitely many solutions of (8) as square prefixes. We call the longest solution maximal. Observe that the maximal solution is not necessarily primitive since any power of a solution to (8) is also a solution. Sturmian words of slope $\alpha$ can be classified into two types. 
Type A. Sturmian words $s$ of slope $\alpha$ which can be written as products of maximal solutions to (8). In other words, it can be written that $s=X_{1}^{2} X_{2}^{2} \cdots$ where $X_{i}$ is the maximal solution occurring as a square prefix of the word $T^{h_{i}}(s)$ where $h_{i}=\left|X_{1}^{2} X_{2}^{2} \cdots X_{i-1}^{2}\right|$.

Type B. Sturmian words $s$ of slope $\alpha$ which are of the form $s=X_{1}^{2} X_{2}^{2} \cdots X_{n}^{2} c$ where $c \in$ $\left\{01 c_{\alpha}, 10 c_{\alpha}\right\}$ and the words $X_{i}$ are maximal solutions as above.

Proposition 6.3 and Lemma 6.2 imply that the words $X_{i}$ in the above definitions are uniquely determined and that the primitive root of a maximal solution is in $\operatorname{RStand}^{+}(\alpha)$. Consequently, a maximal solution is always right special. When finding the factorization of a Sturmian word as a product of squares of maximal solutions, it is sufficient to detect at each position the shortest square of a word in $\operatorname{RStand}^{+}(\alpha)$ and take its largest even power occurring in that position.

Keeping the Sturmian word $s$ of slope $\alpha$ fixed, we define two sequences $\left(\mu_{k}\right)$ and $\left(\lambda_{k}\right)$. We set $\mu_{0}=\lambda_{0}=\varepsilon$. Following the notation above, we define depending on the type of $s$ as follows.

(A) If $s$ is of type $\mathrm{A}$, then we set for all $k \geq 1$ that

$$
\begin{aligned}
& \mu_{k}=X_{1}^{2} X_{2}^{2} \cdots X_{k}^{2} \text { and } \\
& \lambda_{k}=X_{1} X_{2} \cdots X_{k} .
\end{aligned}
$$

(B) If $s$ is of type $\mathrm{B}$, then we set for $1 \leq k \leq n$ that

$$
\begin{aligned}
& \mu_{k}=X_{1}^{2} X_{2}^{2} \cdots X_{k}^{2} \text { and } \\
& \lambda_{k}=X_{1} X_{2} \cdots X_{k},
\end{aligned}
$$

and we let

$$
\begin{aligned}
& \mu_{n+1}=X_{1}^{2} X_{2}^{2} \cdots X_{n}^{2} c \text { and } \\
& \lambda_{n+1}=X_{1} X_{2} \cdots X_{n} c .
\end{aligned}
$$

Compare these definitions with the example in the beginning of this section; the words $X_{1}$ and $X_{2}$ are maximal solutions in the Fibonacci word (which is of type A).

We are finally in a position to formulate precisely the observations made in the beginning of this section and state the main result of this section.

Theorem 6.5. Let s be a Sturmian word with slope $\alpha$.

(A) If $s$ is of type $A$, then

$$
\sqrt{s}=\lim _{k \rightarrow \infty} T^{\left|\lambda_{k}\right|}(s) .
$$

Moreover, the first occurrence of the prefix $\lambda_{k+1}$ of $\sqrt{s}$ is at position $\left|\lambda_{k}\right|$ of $s$ for all $k \geq 0$.

(B) If $s$ is of type $B$, then

$$
\sqrt{s}=T^{\left|\lambda_{n}\right|}(s) \text {. }
$$

Moreover, the first occurrence of the prefix $\lambda_{k+1}$ with $0 \leq k \leq n-1$ is at position $\left|\lambda_{k}\right|$ of s, and the first occurrence of any prefix of $\sqrt{s}$ having lenght greater than $\left|\lambda_{n}\right|$ is at position $\left|\lambda_{n}\right|$ of s.

In particular $\sqrt{s}$ is a Sturmian word with slope $\alpha$.

The theorem only states where the prefixes $\lambda_{k}$ of $\sqrt{s}$ occur for the first time. For the first occurrence of other prefixes of $\sqrt{s}$ we do not have a guaranteed location.

To illustrate the theorem, consider next $\tau$, the eighth shift of the Fibonacci word. If we write under the word $\tau$ each of the corresponding words $\lambda_{k}$ at the position of their first occurrence we get the picture in Figure 2. Theorem 6.5 shows that the nice pattern where the words $\lambda_{k}$ overlap continues indefinitely and, moreover, that if we replace $\tau$ with any other Sturmian word (of type A) we obtain a similar picture. Most of the results of this paper were motivated by the discovery of this pattern.

Before proving the theorem we need one more result. 


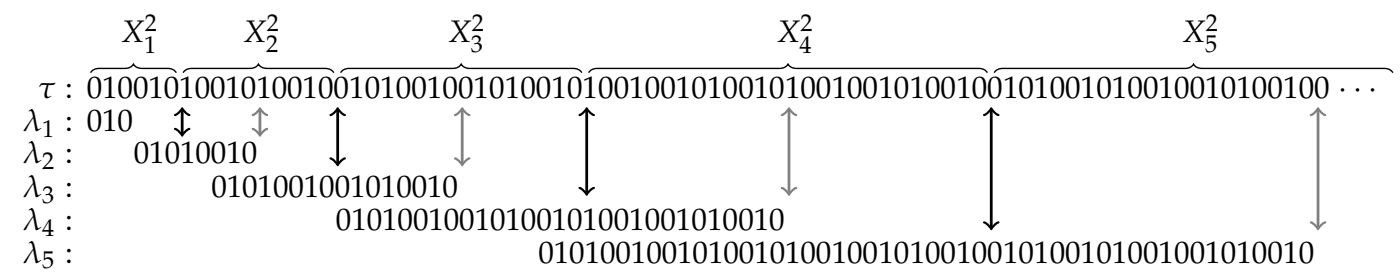

Figure 2: The first occurrences of the words $\lambda_{k}$ in $\tau$. The eighth shift of the Fibonacci word was used since for the Fibonacci word the lengths $\left|\lambda_{k}\right|$ grow very rapidly.

Proposition 6.6. Suppose that $s$ is a Sturmian word of type A. Then the word $\lambda_{k}$ is right special and a suffix of the word $\mu_{k}$ for all $k \geq 0$.

Proof. This proof might be tricky to follow. We advise the reader to keep the picture of Figure 3 in mind while reading the proof. This picture depicts only the Case A below but is surely helpful.

The assertion is evident when $k=0$. Suppose that $k>0$ and assume that $\lambda_{k}$ is right special and that $\lambda_{k}$ is a suffix of the word $\mu_{k}$. It is equivalent to say that $\left\{-\left(\left|\lambda_{k}\right|+1\right) \alpha\right\} \in\left[\lambda_{k}\right]$ and $\left[\mu_{k}\right] \subseteq R^{-\left|\lambda_{k}\right|}\left(\left[\lambda_{k}\right]\right)$ (evidently $\left.2\left|\lambda_{k}\right|=\left|\mu_{k}\right|\right)$. We write simply $\lambda=\lambda_{k}, \mu=\mu_{k}$, and $X=X_{k+1}$. This proof utilizes only the facts that $\mu X^{2} \in \mathcal{L}(\alpha)$ and that $\lambda$ is right special and a suffix of the word $\mu$, not the structure of the words $\lambda$ and $\mu$ implied by their definitions. Thus without loss of generality, we may assume that $X$ is primitive. Consequently, $X \in \operatorname{RStand}^{+}(\alpha)$. It follows that

$$
\begin{aligned}
{[X] } & =I(-(q+1) \alpha, 1-\alpha) \text { and } \\
{\left[X^{2}\right] } & =[X] \cap R^{-|X|}([X])=I(-(q+1) \alpha,-(|X|+1) \alpha) .
\end{aligned}
$$

for some nonnegative integer $q$. Let $x=\{-(|\mu|+1) \alpha\}$. It follows from the hypothesis $\{-(|\lambda|+$ $1) \alpha\} \in[\lambda]$ that $x \in R^{-|\lambda|}([\lambda])$. By (9) the point $x$ is an endpoint of the interval $R^{-|\mu|}([X])$.

Let then $y=\{-(|\mu X|+1) \alpha\}$. By (9) the point $y$ is an endpoint of the interval $R^{-|\mu X|}([X])$ and an interior point of the interval $R^{-|\mu|}([X])$. Suppose for a contradiction that $y \notin R^{-|\lambda|}([\lambda])$. As $x \notin R^{-|\mu X|}([X])$ (otherwise it would follow that $1-\alpha \in R^{-|X|}([X])$ which contradicts (9)), it follows that $R^{-|\lambda|}([\lambda]) \cap R^{-|\mu X|}([X])=\varnothing$. Since

$$
\left[\mu_{k+1}\right]=[\mu] \cap R^{-|\mu|}([X]) \cap R^{-|\mu X|}([X]),
$$

we have that $\left[\mu_{k+1}\right] \subseteq R^{-|\mu X|}([X])$. By assumption $\left[\mu_{k+1}\right] \subseteq[\mu] \subseteq R^{-|\lambda|}([\lambda])$. Thus $\left[\mu_{k+1}\right] \subseteq$ $R^{-|\lambda|}([\lambda]) \cap R^{-|\mu X|}([X])$, so by the above we are forced to conclude that $\left[\mu_{k+1}\right]=\varnothing$. This is a contradiction since $X$ is chosen in such a way that $\left[\mu_{k+1}\right]=\left[\mu X^{2}\right] \neq \varnothing$. We conclude that $y \in R^{-|\lambda|}([\lambda])$.

Now $R^{-|\lambda|}([\lambda X])=R^{-|\lambda|}([\lambda]) \cap R^{-|\mu|}([X])$. Since $y \in R^{-|\lambda|}([\lambda]), R^{-|\mu|}([X])$, it follows that $y=\{-(|\mu X|+1) \alpha\} \in R^{-|\lambda|}([\lambda X])$. Thus $R^{|\lambda|}(y)=\{-(|\lambda X|+1) \alpha\} \in[\lambda X]$, so the word $\lambda X$ is right special. We have two cases depending on the length of the interval $R^{-|\mu|}([X])$ compared to the length of the interval $R^{-|\lambda|}([\lambda])$.

Case A. $R^{-|\mu|}([X]) \nsubseteq R^{-|\lambda|}([\lambda])$. In this case $R^{-|\lambda|}([\lambda X])=I(x, z)$ where $z$ an endpoint of $R^{-|\lambda|}([\lambda])$. Since $y$ is an interior point of $R^{-|\lambda|}([\lambda X]), R^{-|X|}(x)=y$, and $x \notin R^{-|\lambda X|}([\lambda X])$, we obtain that $I(y, z) \subseteq R^{-|\lambda X|}([\lambda X])$. Since $y$ is also an interior point of $R^{-|\lambda|}([\lambda])$, we obtain similarly that $R^{-|\lambda|}([\lambda]) \cap R^{-|\mu|}\left(\left[X^{2}\right]\right)=I(y, z)$. Thus

$$
\left[\mu_{k+1}\right]=[\mu] \cap R^{-|\mu|}\left(\left[X^{2}\right]\right) \subseteq R^{-|\lambda|}([\lambda]) \cap R^{-|\mu|}\left(\left[X^{2}\right]\right)=I(y, z) \subseteq R^{-|\lambda X|}([\lambda X]) .
$$

This proves that $\lambda X=\lambda_{k+1}$ is a suffix of $\mu_{k+1}$. 


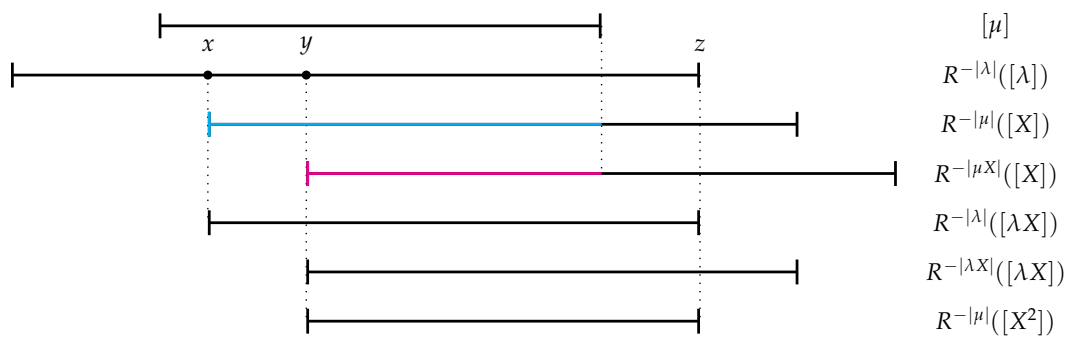

Figure 3: A possible arrangement for the intervals in the Case A of the proof of Proposition 6.6. The blue color marks the interval $[\mu \mathrm{X}]$ and magenta marks the interval $\left[\mu \mathrm{X}^{2}\right]=\left[\mu_{k+1}\right]$.

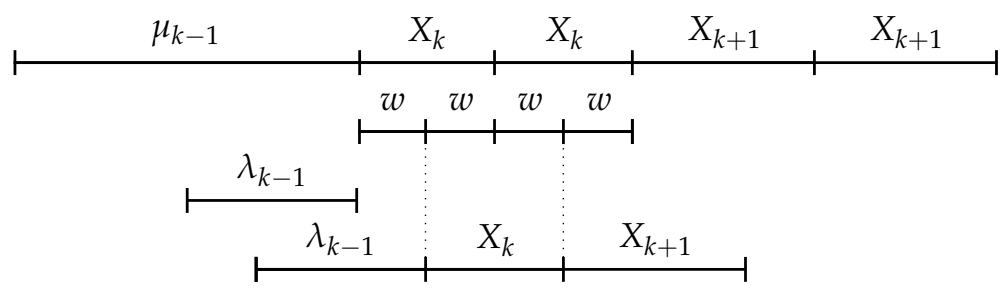

Figure 4: Possible locations for factors in the proof of Theorem 6.5.

Case B. $R^{-|\mu|}([X]) \subseteq R^{-|\lambda|}([\lambda])$. It follows that $R^{-|\lambda|}([\lambda X])=R^{-|\mu|}([X])$, so $R^{-|\lambda X|}([\lambda X])=$ $R^{-|\mu X|}([X])$. Since $R^{-|\bar{\mu}|}\left(\left[X^{2}\right]\right) \subseteq R^{-|\mu X|}([X])$, we get that

$$
\left[\mu_{k+1}\right]=[\mu] \cap R^{-|\mu|}\left(\left[X^{2}\right]\right) \subseteq[\mu] \cap R^{-|\mu X|}([X]) \subseteq[\mu] \cap R^{-|\lambda X|}([\lambda X])
$$

proving that also in this case $\lambda X=\lambda_{k+1}$ is a suffix of $\mu_{k+1}$.

Note that even though $\lambda_{k}$ is right special and always a suffix of $\mu_{k}$, it is not necessary for $\mu_{k}$ to be right special.

Proof of Theorem 6.5. Since Sturmian words of type B differ from Sturmian words of type A essentially only by the fact that the sequence of maximal solutions is finite, it is in this proof enough to consider the case that $s$ is of type A.

Proposition 6.6 says that $\lambda_{k}$ is always a suffix of $\mu_{k}$ for all $k \geq 0$. Since $\left|\mu_{k}\right|=2\left|\lambda_{k}\right|$, it follows that the word $T^{\left|\lambda_{k}\right|}(s)$ has the word $\lambda_{k}$ as a prefix. Therefore $\sqrt{s}=\lim _{k \rightarrow \infty} T^{\left|\lambda_{k}\right|}(s)$.

It remains to prove that the first occurrence of $\lambda_{k+1}$ in $s$ is at position $\left|\lambda_{k}\right|$ of $s$ for all $k \geq 0$. It is clear that the first occurrence of $\lambda_{1}=X_{1}$ is at position $\left|\lambda_{0}\right|=0$. Assume that $k>0$, and suppose for a contradiction that $\lambda_{k+1}$ occurs before the position $\left|\lambda_{k}\right|$. Since $\lambda_{k}$ is a prefix of $\lambda_{k+1}$, by induction we see that $\lambda_{k+1}$ cannot occur before the position $\left|\lambda_{k-1}\right|$. This means that an occurrence of $X_{k} X_{k+1}$ begins in $s$ at position $v$ such that $\left|\mu_{k-1}\right| \leq v<\left|\mu_{k-1} X_{k}\right|$; see Figure 4. Observe that $s$ has at position $\left|\mu_{k-1}\right|$ an occurrence of $X_{k}^{2}$. Write now $X_{k}=w^{t}$ with $w \in \operatorname{RStand}^{+}(\alpha)$. Since $w$ is primitive, we must have that $v=\left|\mu_{k-1}\right|+r|w|$ with $0 \leq r<t$. Thus $X_{k+1}$ occurs in $s$ at position $v+\left|X_{k}\right|=\left|\mu_{k-1}\right|+(r+t)|w|$. Since $r<t$, it follows that either $w$ is a prefix of $X_{k+1}$ or $X_{k+1}$ is a prefix of $w$.

Suppose first that $w$ is a prefix of $X_{k+1}$. If $w=X_{k+1}$, then the prefix $\mu_{k-1} X_{k}^{2}$ of $s$ is followed by $w^{2}$. Now $w^{2 t+2}$ is a solution to (8) implying that $X_{k}$ is not a maximal solution to (8). Since this is contradictory, we infer that $|w|<\left|X_{k+1}\right|$. Since $X_{k+1}$ occurs at position $\left|\mu_{k-1}\right|+(r+t)|w|<\left|\mu_{k}\right|$ and $X_{k+1}$ has $w$ as a prefix, it must be that $X_{k+1}$ begins with $w a$ where $a$ is the first letter of $w$. Since $w$ is right special and $w^{2} \in \mathcal{L}(\alpha)$, it follows that $X_{k+1}^{2}$ begins with $w^{2}$. Like above, this implies that $X_{k}$ is not maximal. This is a contradiction. 
Suppose then that $X_{k+1}$ is a proper prefix of $w$. First of all, $X_{k+1}$ must be primitive, as otherwise $X_{k+1}$ and consequently $w$ would have as a prefix a square of some word in $\operatorname{RStand}^{+}(\alpha)$ contradicting Lemma 6.2. The assumption that $X_{k+1}$ is a prefix of $w$ implies that $X_{k+1}$ and $w$ begin with the same letter. Like above, since $w$ is right special and $w^{2} \in \mathcal{L}(\alpha)$, it must be that $w$ occurs after the prefix $\mu_{k}$ of $s$. Since also $X_{k+1}^{2}$ occurs after the prefix $\mu_{k}$, by Lemma 6.2 we conclude that the word $w$ must be a proper prefix of $X_{k+1}^{2}$. Observe now that the assumption that $X_{k+1}$ is a proper prefix of $w$ excludes the possibilities that $w=\widetilde{s}_{0}=0$ or $w=\widetilde{s}_{1}=10^{a}$. Therefore $w=\widetilde{s}_{h, \ell}$ for some $h \geq 2$ with $0<\ell \leq a_{h}$. Because $|w|<2\left|X_{k+1}\right|$, we must have that $\left|X_{k+1}\right|>\left|\widetilde{s}_{h-2}\right|$. On the other hand, since $\left|X_{k+1}\right|<|w|$ and $X_{k+1}$ and $w$ begin with the same letter, the only option is that $X_{k+1}=\widetilde{s}_{h, \ell^{\prime}}$ with $0<\ell^{\prime}<\ell$. Now

$$
X_{k+1}^{2}=\left(\widetilde{s}_{h-2} \widetilde{s}_{h-1}^{\ell^{\prime}}\right)^{2}=\widetilde{s}_{h-2} \widetilde{s}_{h-1}^{\ell^{\prime}} L\left(\widetilde{s}_{h-1}\right) \widetilde{s}_{h-2} \widetilde{s}_{h-1}^{\ell^{\prime}-1},
$$

so as $w$ is a prefix of $X_{k+1}^{2}$, it must be that $\widetilde{s}_{h-1}=L\left(\widetilde{s}_{h-1}\right)$. This is a contradiction. This final contradiction ends the proof.

As a conclusion of this section, we study the lengths of the maximal solutions of (8). Namely, let $s=X_{1}^{2} X_{2}^{2} \cdots$ be a Sturmian word of type A factorized as a product of maximal solutions $X_{i}$. Computer experiments suggest that typically the sequence $\left(\left|X_{i}\right|\right)$ is strictly increasing. However, there are examples where $\left|X_{i}\right|>\left|X_{i+1}\right|$ for some $i \geq 1$. It is natural to ask if the lengths can decrease significantly or if oscillation is possible. It turns out that neither is possible. In Corollary 6.9 we prove that $\liminf _{i \rightarrow \infty}\left|X_{i}\right|=\infty$.

First we need a result on certain periods of (semi)standard words.

Lemma 6.7. Let $u, v \in \operatorname{Stand}^{+}(\alpha)$ and $|u|>|v|$. If $u$ is a prefix of some word in $v^{+}$, then $u=s_{k, \ell}$ and $v=s_{k-1}$ for some $k \geq 2$ with $0<\ell \leq a_{k}$.

Proof. Suppose that $u$ is a prefix of some word in $v^{+}$. If $u=s_{1}=0^{\mathfrak{a}} 1$, then necessarily $v=s_{0}=0$. Then obviously $u$ is not a prefix of any word in $v^{+}$. Therefore $u=s_{k, \ell}$ for some $k \geq 2$ with $0<\ell \leq a_{k}$. Suppose that $k=2$. Then $u=\left(0^{\mathfrak{a}} 1\right)^{\ell} 0$. It is straightforward to show that $v$ must equal to $s_{1}=0^{\mathfrak{a}} 1$; $u$ cannot be a prefix of a word in $v^{+}$if $v=s_{0}=0$ or $v=s_{2, \ell^{\prime}}$ for some $\ell^{\prime}$ such that $0<\ell^{\prime}<\ell$. Thus we may assume that $k>2$.

Suppose first that $|v|>\left|s_{k-1}\right|$. Then by the assumption $|u|>|v|$, it must be that $v=s_{k, \ell^{\prime}}$ for some $\ell^{\prime}$ such that $\ell^{\prime}<\ell$. Since $u$ is a prefix of some word in $v^{+}$, it follows that the word $w=s_{k-1}^{\ell-\ell^{\prime}} s_{k-2}$ is a prefix of some word in $s_{k-2} v^{+}$. Since the word $w$ begins with $s_{k-1} s_{k-2}$, we obtain that $s_{k-2} v$ begins with $s_{k-1} s_{k-2}$, so $s_{k-1} s_{k-2}=s_{k-2} s_{k-1}$. This is a contradiction.

Assume then that $|v|<\left|s_{k-1}\right|$. Now the prefix $s_{k-1}$ of $u$ is a prefix of some word in $v^{+}$, so by induction $v=s_{k-2}$. Now $u=\left(s_{k-2}^{a_{k-1}} s_{k-3}\right)^{\ell} s_{k-2}$, so as $u$ is a prefix of some word in $v^{+}$, it follows that $z=s_{k-3} s_{k-2}$ is a prefix of some word in $v^{+}$. This means that $z$ ends with a prefix of $s_{k-2}$ of length $\left|s_{k-3}\right|$. As the prefix of $s_{k-2}$ of length $\left|s_{k-3}\right|$ is $s_{k-3}$, the word $z$ ends with $s_{k-3}$. Consequently $s_{k-3} s_{k-2}=s_{k-2} s_{k-3} ;$ a contradiction.

The only remaining option is that $v=s_{k-1}$. This is certainly possible.

The next proposition describes precisely under which conditions it is possible that $\left|X_{i}\right|>$ $\left|X_{i+1}\right|$. Moreover, it rules out the possibility that the lengths decrease significantly or oscillate.

Proposition 6.8. Let $s=X_{1}^{2} X_{2}^{2} X_{3}^{2} \cdots$ be a Sturmian word of type A with slope $\alpha$ factorized as a product of maximal solutions $X_{i}$. If $\left|X_{1}\right|>\left|X_{2}\right|$, then $X_{1}=\widetilde{s}_{k, \ell}$ for some $k \geq 2$ with $0<\ell \leq a_{k}-1$, the primitive root of $X_{2}$ is $\widetilde{s}_{k-1}$, and $\left|X_{3}\right|>\left|X_{1}\right|$.

Proof. Assume that $\left|X_{1}\right|>\left|X_{2}\right|$. Let us first make the additional assumption that $X_{1}$ is primitive. In particular, $X_{1} \in \operatorname{RStand}^{+}(\alpha)$. Let $u$ be the primitive root of $X_{2}$. Then $u \in \operatorname{RStand}^{+}(\alpha)$ and, moreover, by the assumption $\left|X_{1}\right|>\left|X_{2}\right|$ it holds that $|u|<\left|X_{1}\right|$. By Proposition 6.6 the word $\lambda_{2}=X_{1} X_{2}$ is a suffix of the word $\mu_{2}=X_{1}^{2} X_{2}^{2}$. Therefore $X_{1}$ is a proper suffix of $X_{1} X_{2}$, so 
$X_{1} X_{2}=Z X_{1}$ for some nonempty word $Z$. A standard argument shows that $X_{1}$ is a suffix of some word in $X_{2}^{+}$(see e.g., [10, Proposition 1.3.4]). Consequently, $\widetilde{X}_{1}$ is a prefix of a word in $\widetilde{u}^{+}$. As $|u|<\left|X_{1}\right|$, Lemma 6.7 implies that that $X_{1}=\widetilde{s}_{k, \ell}$ and $u=\widetilde{s}_{k-1}$ for some $k \geq 2$ with $0<\ell \leq a_{k}$.

Suppose now that $\ell=a_{k}$. Then the word $X_{1}^{2} X_{2}^{2}$ contains $\widetilde{s}_{k-1} \widetilde{s}_{k-2} \widetilde{s}_{k-1}^{a_{k}+2}$ as a factor. Thus $s_{k-1}^{a_{k}+2} s_{k-2} s_{k-1} \in \mathcal{L}(\alpha)$. As $s_{k-1}$ is a prefix of $s_{k-2} s_{k-1}$, it follows that $s_{k-1}^{a_{k}+3} \in \mathcal{L}(\alpha)$ contradicting Proposition 2.7. Therefore $\ell \leq a_{k}-1$.

Let us then relax the assumption that $X_{1}$ is primitive. Let $v$ be the primitive root of $X_{1}$, so that $X_{1}=v^{j}$ for some $j \geq 1$. Consider now the Sturmian word $T^{(2 j-2)|v|}(s)=v^{2} X_{2}^{2} \cdots$. By the above arguments $v=\widetilde{s}_{k, \ell}$ for some $k \geq 2$ with $0<\ell \leq a_{k}-1$ and the primitive root of $X_{2}$ is $\widetilde{s}_{k-1}$. Further, as $\ell \neq a_{k}$, it follows from Proposition 2.7 that $v^{3} \notin \mathcal{L}(\alpha)$. Thus $j=1$, that is, $X_{1}=\widetilde{s}_{k, \ell}$.

It remains to show that $\left|X_{3}\right|>\left|X_{1}\right|$. Assume for a contradiction that $\left|X_{3}\right| \leq\left|X_{1}\right|$. It is not possible that $\left|X_{3}\right|<\left|X_{2}\right|$ as the preceding arguments show that then $X_{2}$ must be reversed semistandard word; however, $X_{2}$ is a power of the reversed standard word $\widetilde{s}_{k-1}$. Hence by the maximality of $X_{2}$ we have that $\left|X_{3}\right|>\left|X_{2}\right|$. Let $X_{3}=w^{t}$ with $w \in \operatorname{RStand}^{+}(\alpha)$ and $t \geq 1$. As $\left|X_{2}\right|<\left|X_{3}\right| \leq\left|X_{1}\right|$, we have that $\left|s_{k-1}\right|<t|w| \leq\left|s_{k, \ell}\right|$.

Assume for a contradiction that $|w|<\left|s_{k-1}\right|$. If $w$ is semistandard, then Proposition $2.7 \mathrm{im}$ plies that $t=1$, so $t|w|>\left|s_{k-1}\right|$ cannot hold. Thus $w$ is standard. If $w=\widetilde{s}_{0}=0$, then clearly $t|w|>\left|s_{k-1}\right| \geq\left|s_{1}\right|$ cannot hold as the index of the factor 0 in $\mathcal{L}(\alpha)$ is $a+1$. Thus $w \neq \widetilde{s}_{0}$. Suppose first that $w=\widetilde{s}_{k-2}$. Now

$$
t|w|>\left|s_{k-1}\right|=a_{k-1}\left|s_{k-2}\right|+\left|s_{k-3}\right|,
$$

so $t>a_{k-1}$. Since $X_{3}^{2} \in \mathcal{L}(\alpha)$, Proposition 2.7 implies that $2 t \leq a_{k-1}+2$. Therefore

$$
a_{k-1}+2 \geq 2 t>2 a_{k-1}
$$

implying that $a_{k-1}=1$. However, if $a_{k-1}=1$, then $a_{k-1}+2$ is odd, so actually $2 t<a_{k-1}+2$. Then $a_{k-1}+2>2 t>2 a_{k-1}$, so $a_{k-1}<1 ;$ a contradiction. Suppose then that $w=\widetilde{s}_{k-3}$. Now

$$
t|w|>\left|s_{k-1}\right| \geq\left|s_{k-2} s_{k-3}\right|=\left|s_{k-3}^{a_{k-2}} s_{k-4} s_{k-3}\right|>\left(a_{k-2}+1\right)\left|s_{k-3}\right|,
$$

so $t>a_{k-2}+1$. Like previously, as $X_{3}^{2} \in \mathcal{L}(\alpha)$, Proposition 2.7 implies that $2 t \leq a_{k-2}+2$. Like above, we obtain that $a_{k-2}<0$; a contradiction. Similar to above

$$
\left|s_{k-1}\right| \geq\left(a_{k-2}+1\right)\left|s_{k-3}\right|+\left|s_{k-4}\right| \geq 2\left|s_{k-3}\right|+\left|s_{k-4}\right|>\left(2 a_{k-3}+1\right)\left|s_{k-4}\right| .
$$

As $2 a_{k-3}+1 \geq a_{k-3}+2$, we conclude that $\left|s_{k-4}^{a_{k-3}+2}\right|<\left|s_{k-1}\right|$. Therefore by Proposition 2.7 it is not possible that $|w| \leq\left|s_{k-4}\right|$. In conclusion, it is not possible that $t|w|>\left|s_{k-1}\right|$. This is a contradiction.

Now $|w|>\left|\widetilde{s}_{k-1}\right|$ (by the maximality of $X_{2}$ it must be that $w \neq \widetilde{s}_{k-1}$ ). Because $|w| \leq\left|\widetilde{s}_{k, \ell}\right|$, we have that $w=\widetilde{s}_{k, \ell^{\prime}}$ for some $\ell^{\prime}$ such that $0<\ell^{\prime} \leq l$. Since $\ell \neq a_{k}$, the word $w$ is semistandard so by Proposition 2.7 we have that $t=1$. By Proposition 6.6 the word $\lambda_{3}=X_{1} X_{2} X_{3}$ is a suffix of the word $\mu_{3}=X_{1}^{2} X_{2}^{2} X_{3}^{2}$. It follows that $\widetilde{s}_{k-2} \widetilde{s}_{k-1}^{\ell+r}=\widetilde{s}_{k-1}^{\ell+r-\ell^{\prime}} \widetilde{s}_{k-2} \widetilde{s}_{k-1}^{\ell^{\prime}}$ where $r$ is such that $X_{2}=\widetilde{s}_{k-1}^{r}$. Therefore the words $\widetilde{s}_{k-2}$ and $\widetilde{s}_{k-1}$ commute; a contradiction. This final contradiction proves that $\left|X_{3}\right|>\left|X_{1}\right|$.

Corollary 6.9. Let $s=X_{1}^{2} X_{2}^{2} \cdots$ be a Sturmian word of type A with slope $\alpha$ factorized as a product of maximal solutions $X_{i}$. Then $\lim _{i n f} \operatorname{in}_{i \rightarrow \infty}\left|X_{i}\right|=\infty$.

Proof. This follows from Proposition 6.8: if $\left|X_{i+1}\right|<\left|X_{i}\right|$ for some $i \geq 1$, then $\left|X_{i+2}\right|>\left|X_{i}\right|$. 


\section{The Square Root of the Fibonacci Word}

In this section we prove a formula for the square root of the Fibonacci word. To do this we factorize the Fibonacci word as a product of maximal solutions to (8).

We denote by $\Phi$ the slope of the Fibonacci word, that is, $\Phi=[0 ; 2,1,1, \ldots]$. Further, we set

$$
t_{k}= \begin{cases}01, & \text { if } k \text { is even } \\ 10, & \text { if } k \text { is odd }\end{cases}
$$

We need two lemmas specific to the slope $\Phi$.

Lemma 7.1. For the standard words of slope $\Phi$ it holds that $t_{k} s_{k} s_{k+1} s_{k+2}=\widetilde{s}_{k+2}^{2} t_{k+1}$ for all $k \geq 0$.

Proof. The case $k=0$ is verified directly: $t_{0} s_{0} s_{1} s_{2}=01 \cdot 0 \cdot 01 \cdot 010=(010)^{2} \cdot 10=\widetilde{s}_{2}^{2} t_{1}$. Let then $k \geq 1$. There exists a palindrome $P_{k}$ such that $s_{k}=P_{k} \widetilde{t}_{k}$ for all $k \geq 1$ (see e.g. [11, Lemma 2.2.8]). Now

$$
\begin{aligned}
t_{k} s_{k} s_{k+1} s_{k+2} & =t_{k} P_{k} \widetilde{t}_{k} s_{k+1} s_{k+2}=\widetilde{s}_{k} \widetilde{t}_{k} P_{k+1} \widetilde{t}_{k+1} s_{k+2}=\widetilde{s}_{k} t_{k+1} P_{k+1} \widetilde{t}_{k+1} s_{k+2} \\
& =\widetilde{s}_{k} \widetilde{s}_{k+1} t_{k+1} P_{k+2} \widetilde{t}_{k+2}=\widetilde{s}_{k} \widetilde{s}_{k+1} \widetilde{s}_{k+2} \widetilde{t}_{k+2}=\widetilde{s}_{k+2}^{2} t_{k+1},
\end{aligned}
$$

which proves the claim.

Lemma 7.2. For the standard words of slope $\Phi$ it holds that $s_{3 k+4}=\prod_{i=0}^{k} \widetilde{s}_{3 i+2}^{2} \cdot t_{k+1}$ for all $k \geq 0$.

Proof. If $k=0$, then $s_{4}=01001010=\widetilde{s}_{2}^{2} t_{1}$. Let then $k \geq 1$. Now

$$
\begin{aligned}
s_{3 k+4} & =s_{3 k+3} s_{3 k+2}=s_{3 k+2} s_{3 k+1} s_{3 k+2}=s_{3 k+1} s_{3 k} s_{3 k+1} s_{3 k+2} \\
& =s_{3(k-1)+4} s_{3 k} s_{3 k+1} s_{3 k+2}=\prod_{i=0}^{k-1} \widetilde{s}_{3 i+2}^{2} \cdot t_{k} s_{3 k} s_{3 k+1} s_{3 k+2}
\end{aligned}
$$

where the last equality follows by induction. By applying Lemma 7.1 we obtain that

$$
s_{3 k+4}=\prod_{i=0}^{k-1} \widetilde{s}_{3 i+2}^{2} \cdot \widetilde{s}_{3 k+2}^{2} t_{k+1}=\prod_{i=0}^{k} \widetilde{s}_{3 i+2}^{2} \cdot t_{k+1},
$$

which proves the claim.

As an immediate corollary to Lemma 7.2 we obtain a formula for the square root of the Fibonacci word.

Theorem 7.3. For slope $\Phi$ we have that

$$
c_{\Phi}=\prod_{i=0}^{\infty} \widetilde{s}_{3 i+2}^{2} \quad \text { and } \quad \sqrt{c_{\Phi}}=s_{\frac{1}{2}, \Phi}=\prod_{i=0}^{\infty} \widetilde{s}_{3 i+2} .
$$

The preceding arguments are very specific to the Fibonacci word. The reader might wonder if formulas for the square roots of other standard Sturmian words exist. Surely, for some specific words such formulas can be derived, but we believe no general factorization for the square roots of standard Sturmian words can be given. Let us give some arguments supporting our belief.

Let $s=X_{1}^{2} X_{2}^{2} \cdots$ be a standard Sturmian word of slope $\alpha$ factorized as a product of maximal solutions to (8). The word $s$ begins with the word $0^{\mathfrak{a}} 1$. Therefore if $\mathfrak{a}>1$, then $X_{1}=0^{\lfloor\mathfrak{a} / 2\rfloor}$. Thus if $\mathfrak{a}>1$, then $X_{2}$ begins with 0 if and only if $\mathfrak{a}$ is odd. Because of the asymmetry of the letters 0 and 1 in the minimal squares of slope $\alpha(1)$, the parity of the parameter $\mathfrak{a}$ greatly influences the remaining words $X_{i}$. Moreover, it is not just the partial quotient $a_{1}$ which influences the 


\begin{tabular}{|c|c|c|c|}
\hline$a_{4} a_{3}$ & 1 & 2 & 3 \\
\hline 1 & $2,5,8$ & $2,4,7$ & $2,5,8$ \\
\hline 2 & $2,3,6$ & $2,4,7$ & $2,3,6$ \\
\hline 3 & $2,3,5$ & $2,4,7$ & $2,3,5$ \\
\hline
\end{tabular}

Table 1: How $X_{1}, X_{2}$, and $X_{3}$ are affected when $a_{3}$ and $a_{4}$ vary in the case that $a_{1}=2$ and $a_{2}=1$.

\begin{tabular}{|c|c|c|c|}
\hline$a_{4} a_{3}$ & 1 & 2 & 3 \\
\hline 1 & 1 & $\overline{0}$ & 1 \\
\hline 2 & 1 & 0 & 1 \\
\hline 3 & 0 & 0 & 0 \\
\hline
\end{tabular}

Table 2: How the first letter of $X_{4}$ varies when $a_{3}$ and $a_{4}$ vary in the case that $a_{1}=2$ and $a_{2}=1$.

factorization. Suppose for instance that $a_{1}=2$ and $a_{2}=1$. Table 1 shows how the values of the partial quotients $a_{3}$ and $a_{4}$ affect the words $X_{i}$. The cell of the table tells to which squares of reversed standard words the words $X_{1}, X_{2}$ and $X_{3}$ correspond to. For example if $a_{3}=2$ and $a_{4}=1$, then the standard Sturmian word of slope $[0 ; 2,1,2,1, \ldots]$ begins with $\widetilde{s}_{2}^{2} \widetilde{s}_{4}^{2} \widetilde{s}_{7}^{2}$. Table 2 tells the first letter of the corresponding word $X_{4}^{2}$. As can be observed from Table 2, the first letter of $X_{4}^{2}$ varies when $a_{3}$ and $a_{4}$ vary. Because of the asymmetry, it is thus expected that slight variation in partial quotients drastically changes the factorization as a product of maximal solutions to (8). Since similar behavior is expected from the rest of the partial quotients, it seems to us that no nice formula (like e.g., the formula of Theorem 7.3) can be given for the square root of a standard Sturmian word in terms of reversed standard words.

De Luca and Fici proved a nice formula for a certain shift of a standard Sturmian word [4, Theorem 18].

Proposition 7.4. Let $c_{\alpha}$ be the standard Sturmian word of slope $\alpha=[0 ; \mathfrak{a}+1, \mathfrak{b}+1, \ldots]$. Then

$$
c_{\alpha}=0^{\mathfrak{a}} 10^{\mathfrak{a}-1} \prod_{k=1}^{\infty} \widetilde{s}_{k}^{2}
$$

As a corollary of this theorem we obtain that the word $\sqrt{T^{2 \mathfrak{a}\left(c_{\alpha}\right)}}=\prod_{k=1}^{\infty} \widetilde{s}_{k}$ is a Sturmian word of slope $\alpha$ with intercept $\psi(\{(2 \mathfrak{a}+1) \alpha\})=\mathfrak{a} \alpha$. We have thus shown that

$$
c_{\alpha}=0^{\mathfrak{a}-1} \prod_{k=1}^{\infty} \widetilde{s}_{k} .
$$

In particular, we obtain the well-known result that the Fibonacci infinite word is a product of the reversed Fibonacci words.

\section{A Curious Family of Subshifts}

In this section we construct a family of linearly recurrent and optimal squareful words which are not Sturmian but are fixed points of the (more general) square root map. Moreover, we show that any subshift $\Omega$ generated by such a word has a curious property: for every $w \in \Omega$ either $\sqrt{w} \in \Omega$ or $\sqrt{w}$ is periodic.

It is evident from Proposition 2.2 that Sturmian words are a proper subclass of optimal squareful words. As Sturmian words have the exceptional property that their language is preserved under the square root map, it is natural to ask if other optimal squareful words can have this 
property. We show that, indeed, such words exist by an explicit construction. The idea behind the construction is to mimic the structure of the Sturmian words $01 c_{\alpha}$ and $10 c_{\alpha}$. The simple reason why these words are fixed points of the square root map (thus preserving the language) is that they have arbitrarily long squares of solutions to (8) as prefixes. Thus to obtain a fixed point of the square root map, it is sufficient to find a sequence $\left(u_{k}\right)$ of solutions to (8) with the property that $u_{k}^{2}$ is a proper prefix of $u_{k+1}^{2}$ for all $k \geq 1$. Let us show how such a sequence can be obtained.

Let $S$ be a fixed primitive solution to (8) in the language of some Sturmian word with slope $[0 ; \mathfrak{a}+1, \mathfrak{b}+1, \ldots]$ such that $|S|>\left|S_{6}\right|$. In particular, $S$ has the word $S_{6}=10^{\mathfrak{a}+1}\left(10^{\mathfrak{a}}\right)^{\mathfrak{b}+1}$ as a proper suffix. Recall from the proof of Lemma 5.6 that $|S| \geq\left|S_{5} S_{6}\right|$. We denote the word $L(S)$ simply by $L$. Using the word $S$ as a seed solution, we produce a sequence $\left(\gamma_{k}\right)$ of primitive solutions to (8) defined by the recurrence

$$
\gamma_{1}=S, \quad \gamma_{k+1}=L\left(\gamma_{k}\right) \gamma_{k}^{2} \quad \text { for } k \geq 2
$$

We need to prove that the sequence $\left(\gamma_{k}\right)$ really is a sequence of primitive solutions to (8). Before showing this, let us define

$$
\Gamma_{1}=\lim _{k \rightarrow \infty} \gamma_{2 k} \quad \text { and } \quad \Gamma_{2}=\lim _{k \rightarrow \infty} \gamma_{2 k+1}
$$

The limits exist as $\gamma_{k}^{2}$ is always a prefix of $\gamma_{k+2}$. Hence both $\Gamma_{1}$ and $\Gamma_{2}$ have arbitrarily long squares of words in the sequence $\left(\gamma_{k}\right)$ as prefixes. Observe also that $\mathcal{L}\left(\Gamma_{1}\right)=\mathcal{L}\left(\Gamma_{2}\right)$. As there is not much difference between $\Gamma_{1}$ and $\Gamma_{2}$ in terms of structure, we set $\Gamma$ to be either of these words.

Taking for granted that the sequence $\left(\gamma_{k}\right)$ is a sequence of solutions to (8), we see that $\sqrt{\Gamma}=\Gamma$. Note that we also need to ensure that the word $\Gamma$ is optimal squareful for the square root map to make sense.

Next we aim to prove the following.

Proposition 8.1. The word $\gamma_{k}$ is a primitive solution to (8) in $\mathcal{L}(\mathfrak{a}, \mathfrak{b})$ for all $k \geq 1$.

Recall from Section 5 that the language $\mathcal{L}(\mathfrak{a}, \mathfrak{b})$ consists of all factors of the infinite words in the language

$$
\left(10^{\mathfrak{a}+1}\left(10^{\mathfrak{a}}\right)^{\mathfrak{b}}+10^{\mathfrak{a}+1}\left(10^{\mathfrak{a}}\right)^{\mathfrak{b}+1}\right)^{\omega}=\left(S_{5}+S_{6}\right)^{\omega} .
$$

Before we can prove Proposition 8.1, we need to know that the words $\gamma_{k}$ are primitive and that they are factors of some optimal squareful word with parameters $\mathfrak{a}$ and $\mathfrak{b}$.

Lemma 8.2. The word $\gamma_{k}$ is primitive for all $k \geq 1$.

Proof. We proceed by induction. By definition $\gamma_{1}$ is primitive. Let $k \geq 1$, and suppose for a contradiction that $\gamma_{k+1}$ is not primitive; that is, $\gamma_{k+1}=L\left(\gamma_{k}\right) \gamma_{k}^{2}=z^{n}$ for some primitive word $z$ and $n>1$. If $n=2$, then obviously $\left|\gamma_{k}\right|$ must be even, and the suffix of $\gamma_{k}$ of length $\left|\gamma_{k}\right| / 2$ must be a prefix of $\gamma_{k}$. This contradicts the primitivity of $\gamma_{k}$. The case $n=3$ would clearly imply that $\gamma_{k}=L\left(\gamma_{k}\right)$, which is not possible. Hence $n>3$, and further $|z|<\left|\gamma_{k}\right|$. As $\gamma_{k}^{2}$ is a suffix of some word in $z^{+}$, it follows that $z=u v$ where $v u$ is a suffix of $\gamma_{k}$. On the other hand, $z$ is a suffix of $\gamma_{k}$, so $u v=v u$. Since $z$ is primitive, the only option is that $u$ is empty. Therefore $\gamma_{k} \in z^{+}$; a contradiction with the primitivity of $\gamma_{k}$.

Lemma 8.3. We have that $\gamma_{k}, L\left(\gamma_{k}\right) \in \mathcal{L}(\mathfrak{a}, \mathfrak{b})$ for all $k \geq 1$.

Proof. For a suitable slope $\alpha=[0 ; \mathfrak{a}+1, \mathfrak{b}+1, \ldots]$, either of the words $S$ and $L$ is a reversed standard word of slope $\alpha$. Thus by Theorem 5.2 both $S^{2}$ and $L^{2}$ are in $\mathcal{L}(\alpha)$, so $S^{2}, L^{2} \in \mathcal{L}(\mathfrak{a}, \mathfrak{b})$.

We clearly have that $\gamma_{1} \in \mathcal{L}(\mathfrak{a}, \mathfrak{b})$. Note that by the assumption $|S|>\left|S_{6}\right|$ both of the words $S$ and $L$ have the word $s=S_{6}=10^{\mathfrak{a}+1}\left(10^{\mathfrak{a}}\right)^{\mathfrak{b}+1}$ as a proper suffix. Write $S=u s$. Since $s$ begins with $10^{\mathfrak{a}+1}$ and $S^{2}$ has sus as a suffix, it follows that $u s \in\left(S_{5}+S_{6}\right)^{+}$. Using the fact that $L \in \mathcal{L}(\mathfrak{a}, \mathfrak{b})$, 
we see that $\gamma_{2}=L S S=L(u) s(u s)^{2} \in \mathcal{L}(\mathfrak{a}, \mathfrak{b})$. Clearly $L\left(\gamma_{2}\right)=S^{3}=(u s)^{3} \in \mathcal{L}(\mathfrak{a}, \mathfrak{b})$. Proceeding by induction we may assume that $k \geq 2$ and $\gamma_{k}, L\left(\gamma_{k}\right) \in \mathcal{L}(\mathfrak{a}, \mathfrak{b})$. Since $\gamma_{k}$ has either $S$ or $L$ as a prefix, it can be written that $\gamma_{k}=v s z s$ with $|v s|=|S|$. It follows that $s z \in\left(S_{5}+S_{6}\right)^{+}$. Since svs is a suffix of either $S^{2}$ or $L^{2}$, we have that $s v \in\left(S_{5}+S_{6}\right)^{+}$. Therefore $s v s z \in\left(S_{5}+S_{6}\right)^{+}$. As $L\left(\gamma_{k}\right)=$ $L(v s z) s \in \mathcal{L}(\mathfrak{a}, \mathfrak{b})$, we have that $L(v s z)$ is a suffix of some word in $\left(S_{5}+S_{6}\right)^{+}$. Overall, the word $\gamma_{k+1}=L(v s z)(s v s z)^{2} s$ is in $\mathcal{L}(\mathfrak{a}, \mathfrak{b})$. Clearly then must the word $L\left(\gamma_{k+1}=(v s z s)^{3}=v s z(s v s z)^{2} s\right.$ also be in $\mathcal{L}(\mathfrak{a}, \mathfrak{b})$.

Note that without the assumption $|S|>\left|S_{6}\right|$ the conclusion of the above lemma fails to hold. If $S=S_{6}=10^{\mathfrak{a}+1}\left(10^{\mathfrak{a}}\right)^{\mathfrak{b}+1}$, then $L=0\left(10^{\mathfrak{a}}\right)^{\mathfrak{b}+2}$ and $L S=0\left(10^{\mathfrak{a}}\right)^{\mathfrak{b}+2} 10^{\mathfrak{a}+1}\left(10^{\mathfrak{a}}\right)^{\mathfrak{b}+1}$. Therefore $L S \notin \mathcal{L}(\mathfrak{a}, \mathfrak{b})$, and consequently $\gamma_{2}=L S^{2} \notin \mathcal{L}(\mathfrak{a}, \mathfrak{b})$.

Proof of Proposition 8.1. We proceed by induction. By Lemma 8.2 the word $\gamma_{k}$ is primitive for all $k \geq 1$. Lemma 8.3 tells that both of the words $\gamma_{k}$ and $L\left(\gamma_{k}\right)$ are in $\mathcal{L}(\mathfrak{a}, \mathfrak{b})$ for all $k \geq 1$. By definition both $\gamma_{1}$ and $L\left(\gamma_{1}\right)$ are solutions to (8). We may thus assume that $k \geq 1$ and both $\gamma_{k}$ and $L\left(\gamma_{k}\right)$ are solutions to (8). It follows from Lemma 5.6 that

$$
\gamma_{k} L\left(\gamma_{k}\right) \in \Pi(\mathfrak{a}, \mathfrak{b}) \text { and } \sqrt{\gamma_{k} L\left(\gamma_{k}\right)}=\gamma_{k} .
$$

Since $L\left(\gamma_{k}\right)$ is a solution to (8), Lemma 5.6 also implies that

$$
L\left(\gamma_{k}\right) \gamma_{k} \in \Pi(\mathfrak{a}, \mathfrak{b}) \text { and } \sqrt{L\left(\gamma_{k}\right) \gamma_{k}}=L\left(\gamma_{k}\right)
$$

Because

$$
\gamma_{k+1}^{2}=L\left(\gamma_{k}\right) \gamma_{k} \cdot \gamma_{k} L\left(\gamma_{k}\right) \cdot \gamma_{k}^{2}
$$

we obtain that

$$
\gamma_{k+1}^{2} \in \Pi(\mathfrak{a}, \mathfrak{b}) \text { and } \sqrt{\gamma_{k+1}^{2}}=\sqrt{L\left(\gamma_{k}\right) \gamma_{k}} \sqrt{\gamma_{k} L\left(\gamma_{k}\right)} \sqrt{\gamma_{k}^{2}}=L\left(\gamma_{k}\right) \gamma_{k} \gamma_{k}=\gamma_{k+1} .
$$

This proves that $\gamma_{k+1}$ is a solution to (8). Consider next the word $L\left(\gamma_{k+1}\right)=\gamma_{k}^{3}$. Because $\left(L\left(\gamma_{k+1}\right)\right)^{2}=\left(\gamma_{k}^{2}\right)^{3}$, it is evident that

$$
\left(L\left(\gamma_{k+1}\right)\right)^{2} \in \Pi(\mathfrak{a}, \mathfrak{b}) \text { and } \sqrt{\left(L\left(\gamma_{k+1}\right)\right)^{2}}=\gamma_{k}^{3}=L\left(\gamma_{k+1}\right) .
$$

Therefore also $L\left(\gamma_{k+1}\right)$ is a solution to (8). The conclusion follows.

As we remarked earlier, we have now proved that $\Gamma$ is a fixed point of the square root map. Next we show that the word $\Gamma$ is aperiodic, linearly recurrent, and not Sturmian.

Lemma 8.4. The word $\gamma_{2}^{2}$ is not a factor of any Sturmian word.

Proof. By definition $\gamma_{2}=L S^{2}$. Write $S=x y w$ and $L=y x w$ for some word $w$ and distinct letters $x$ and $y$. Now $\gamma_{2}^{2}=x y x w(x y w)^{2} y x w(x y w)^{2}$, so the word $\gamma_{2}^{2}$ has factors $x w x$ and $y w y$. Hence $\gamma_{2}^{2}$ is not balanced, and it cannot be a factor of any Sturmian word.

Lemma 8.5. The word $\Gamma$ is aperiodic and linearly recurrent.

Proof. The recurrence (10) and the definition (11) of $\Gamma$ show that for all $k \geq 1$ the word $\Gamma$ is a product of the words $\gamma_{k+1}=L\left(\gamma_{k}\right) \gamma_{k}^{2}$ and $L\left(\gamma_{k+1}\right)=\gamma_{k}^{3}$ such that between two occurrences of $L\left(\gamma_{k+1}\right)$ there is always $\gamma_{k}^{2}$ or $\gamma_{k}^{5}$. From this it follows that the return time of a factor of $\Gamma$ of length $\gamma_{k}$ is at most the return time of the factor $L\left(\gamma_{k}\right)$, which is at most $6\left|\gamma_{k}\right|$. Let then $w$ be a factor of $\Gamma$ such that $\left|\gamma_{k}\right|<|w| \leq\left|\gamma_{k+1}\right|$. Since $w$ is a factor of some factor of $\Gamma$ of length $\left|\gamma_{k+1}\right|$, it follows 
that the return time of $w$ is at most $6\left|\gamma_{k+1}\right|$. Now $6\left|\gamma_{k+1}\right|=18\left|\gamma_{k}\right|<18|w|$ proving that $\Gamma$ is linearly recurrent.

The preceding shows that $\gamma_{k}$ is followed in $\mathcal{L}(\Gamma)$ by both $\gamma_{k}$ and $L\left(\gamma_{k}\right)$. As the first letters of $\gamma_{k}$ and $L\left(\gamma_{k}\right)$ are distinct, the factor $\gamma_{k}$ is right special. Thus $\mathcal{L}(\Gamma)$ contains arbitrarily long right special factors, so $\Gamma$ must be aperiodic.

Since linearly recurrent words have linear factor complexity [5, Theorem 24], it follows from Lemma 8.5 that $\Gamma$ has linear factor complexity.

We observed in the previous proof that the word $\Gamma$ is a product of the words $S$ and $L$ such that between two occurrences of $L$ in this product there is always $S^{2}$ or $S^{5}$. Since $S$ and $L$ are primitive, any word $w \in \mathcal{L}(\Gamma)$ which is a product of the words $S$ and $L$ such that $|w| \geq 6|S|$ must synchronize to the factorization of $\Gamma$ as a product of the words $S$ and $L$. That is, for any factorization $\Gamma=u w \Gamma^{\prime}$ we must have that $|u|$ is a multiple of $|S|$.

Theorem 8.6. The word $\Gamma$ is a non-Sturmian, linearly recurrent optimal squareful word which is a fixed point of the square root map.

Proof. The fact that $\Gamma$ is optimal squareful and linearly recurrent follows from Lemmas 8.3 and 8.5. The argument outlined at the beginning of this section shows that $\Gamma$ is a fixed point of the square root map as by Proposition 8.1 the words $\gamma_{k}$ which occur as square prefixes in $\Gamma$ are solutions to (8). Finally, $\Gamma$ contains the factor $\gamma_{2}^{2}$, so $\Gamma$ is not Sturmian by Lemma 8.4.

Denote by $\Omega$ the subshift consisting of the infinite words having language $\mathcal{L}(\Gamma)$. As $\Gamma$ is linearly recurrent, it is uniformly recurrent, so the subshift $\Omega$ is minimal. The rest of this section is devoted to proving the result mentioned in the beginning of this section.

Theorem 8.7. For all $w \in \Omega$ either $\sqrt{w} \in \Omega$ or $\sqrt{w}$ is (purely) periodic with minimal period conjugate to $S$. Moreover, there exists words $u, v \in \Omega$ such that $\sqrt{u} \in \Omega$ and $\sqrt{v}$ is periodic.

This result is very surprising since it is contrary to the plausible hypothesis that an aperiodic word must map to an aperiodic word under the square root map.

It is not difficult to prove Theorem 8.7 for words in $\Omega$ which are products of the words $S$ and $L$. We prove this special case next in Lemma 8.8. However, difficulties arise since a word in $\Omega$ can start in an arbitrary position of an infinite product of $S$ and $L$. There are certain well-behaved positions in $S$ and $L$ which are easier to handle. Theorem 8.7 is proved for these special positions in Lemma 8.10. The rest of the effort is in demonstrating that all the other cases can be reduced to these well-behaved cases. We begin by proving the easier cases, and we conclude with the reductions.

Lemma 8.8. If a word $w \in \Omega$ can be written as a product of the words $S$ and $L$, then $\sqrt{w} \in \Omega$.

Proof. Any word $u$ which is a product of the words $S$ and $L$ can be naturally written as a binary word $\bar{u}$ over the alphabet $\{S, L\}$. If such a word $\bar{u}$ has even length, then it is a word over the alphabet $A=\{S S, S L, L S, L L\}$. Using the fact that $\sqrt{S S}=S, \sqrt{S L}=S, \sqrt{L S}=L$, and $\sqrt{L L}=L$ (see Lemma 5.6), we can define a square root for a word over $A$.

The word $\gamma_{k}^{2}$ is a prefix of $\Gamma$ for all $k \geq 1$. Thus $\gamma_{k}$ has occurrences at positions 0 and $\left|\gamma_{k}\right|$ of $\Gamma$. Clearly $\left|\gamma_{k}\right|=3^{k-1}|S|$, so the word $\bar{\gamma}_{k}$ occurs in $\bar{\Gamma}$ in an even and in an odd position.

Let $v$ be a prefix of $w$ of length $|v|=2 n|S|$ for some $n \geq 1$, so $\bar{v}$ is a word over $A$. Since $v$ is a prefix of $w$, the word $v$ is a factor of some $\gamma_{k}$. Since $\bar{\gamma}_{k}$ occurs in $\bar{\Gamma}$ in an even and in an odd position, the word $\bar{v}$ occurs in an even position in $\bar{\Gamma}$. Hence $\bar{\Gamma}$ can be factored as $\bar{\Gamma}=z \bar{v} t$ where $z$ and $t$ are finite or infinite words over $A$. Since $\Gamma$ is a fixed point of the square root map, we have that $\bar{\Gamma}=\sqrt{z} \sqrt{\bar{v}} \sqrt{t}$. Hence $\sqrt{v} \in \mathcal{L}(\Gamma)$. It follows that $\mathcal{L}(\sqrt{w}) \subseteq \mathcal{L}(\Gamma)$, so $\sqrt{w} \in \Omega$. 
Definition 8.9. Let $w$ be a word and $\ell$ be an integer such that $0<\ell<|w|$. If the factor of $w^{3}$ of length $\left|w^{2}\right|$ starting at position $\ell$ can be written as a product of minimal squares $X_{1}^{2}, \ldots, X_{n}^{2}$, then we say that the position $\ell$ of $w$ is repetitive. If in addition $\left|X_{1}^{2} \cdots X_{m}^{2}\right| \neq|w|-\ell,\left|w^{2}\right|-\ell$ for all $m$ such that $1 \leq m \leq n$, then we say that the position $\ell$ is nicely repetitive.

For example if $\mathfrak{a}=1, \mathfrak{b}=0$, and $S=1001001010010$, then the position 1 of $S$ is repetitive as the factor 00100101001010010010100101 of $S^{3}$ of length $\left|S^{2}\right|=26$ starting at position 1 is in $\Pi(\mathfrak{a}, \mathfrak{b})$. This position is not nicely repetitive as $\left|0^{2} \cdot(10010)^{2}\right|=12=|S|-1$. The position 2 of $S$, however, can be checked to be nicely repetitive. The position 4 of $S$ is not repetitive as the factor 00101001010010010100101001 of length 26 starting at position 4 is not in $\Pi(\mathfrak{a}, \mathfrak{b})$.

In the upcoming proof of Theorem 8.7 we will show that if $w \in \Omega$ is a product of the words $S$ and $L$ and $\ell$ is a nicely repetitive position of $S$, then the word $\sqrt{T^{\ell}(w)}$ is always periodic. On the other hand, we show that if $\ell$ is not a nicely repetitive position then $\sqrt{T^{\ell}(w)}$ is always in $\Omega$.

Next we identify some good positions in the suffix $S_{6}$ of $S$. As we observed in the proof of Lemma 5.6, the suffix $S_{6}$ of $S$ restricts locally how a factorization of a word as a product of minimal squares continues after an occurrence of $S_{6}$. Consider a product $X_{1}^{2} \cdots X_{n}^{2}$ of minimal squares which has an occurrence of $S_{6}$ at position $\ell$. Then for some $m \in\{1, \ldots, n\}$ the minimal square $X_{m}^{2}$ must begin at some of the positions $\ell, \ell+1, \ldots, \ell+\left|S_{6}\right|-1$. Otherwise some minimal square would have $S_{6}$ as an interior factor; yet no such minimal square exists. Among the positions $\ell, \ell+1, \ldots, \ell+\left|S_{6}\right|-1$ we are interested in the largest position where a minimal square may begin. Let

$$
\mathcal{B}=\left\{\ell \in\left\{0, \ldots,\left|S_{6}\right|-1\right\}: \text { no square of length at most }\left|S_{6}\right|-\ell \text { begins at position } \ell \text { of } S_{6}\right\} .
$$

It is straightforward to see that

$$
\mathcal{B}=\left\{\left|S_{6}\right|-\left|S_{6}\right|,\left|S_{6}\right|-\left|S_{4}\right|,\left|S_{6}\right|-\left|S_{3}\right|,\left|S_{6}\right|-\left|S_{1}\right|\right\} .
$$

We are interested in those positions of the suffix $S_{6}$ of $S$ where no minimal square begins. Hence we define

$$
\mathcal{B}_{S}=\left\{\ell: \ell-|S|+\left|S_{6}\right| \in \mathcal{B}\right\}=\left\{|S|-\left|S_{6}\right|,|S|-\left|S_{4}\right|,|S|-\left|S_{3}\right|,|S|-\left|S_{1}\right|\right\} .
$$

A consequence of the definitions is that if $\ell$ is a position of $S$ such that $\ell \notin \mathcal{B}_{S}$, then there exists $\ell^{\prime} \in \mathcal{B}_{S} \cup\{|S|\}$ such that $S\left[\ell, \ell^{\prime}-1\right] \in \Pi(\mathfrak{a}, \mathfrak{b})$. This fact is used later several times.

Lemma 8.10. Suppose that $w \in \Omega$ can be written as a product of the words $S$ and $L$. Assume that the position $\ell \in \mathcal{B}_{S}$ is nicely repetitive. Let the prefix of $T^{\ell}(w)$ of length $\left|S^{2}\right|$ be factorized as a product of minimal squares $X_{1}^{2} \cdots X_{n}^{2}$. Then the word $\sqrt{T^{\ell}(w)}$ is periodic with minimal period $X_{1} \cdots X_{n}$. Moreover, $X_{1} \cdots X_{n}$ is conjugate to $S$.

Proof Sketch. As $\ell$ is repetitive, the factor $u$ of length $\left|S^{2}\right|$ of $S^{3}$ starting at position $\ell$ is in $\Pi(\mathfrak{a}, \mathfrak{b})$. If we substitute the middle $S$ in $S^{3}$ with $L$, then an application of Lemma 5.5 shows that the factor of length $\left|S^{2}\right|$ of $S L S$ starting at position $\ell$ is still in $\Pi(\mathfrak{a}, \mathfrak{b})$ and that the square root of this factor coincides with the square root of $u$ (here we need that $\ell \in \mathcal{B}_{S}$ ). Further analysis shows that if we substitute the words $S$ in $S^{3}$ in any way, then the square root of the factor of length $\left|S^{2}\right|$ beginning at position $\ell$ is unaffected. Since $\ell$ is repetitive, the prefix of $T^{\ell+\left|S^{2}\right|}(w)$ of length $\left|S^{2}\right|$ is again in $\Pi(\mathfrak{a}, \mathfrak{b})$ and has the same square root, and so on. Thus $\sqrt{T^{\ell}(w)}$ is periodic. Since both the square of the period and $S^{2}$ occur in a suitable Sturmian word; having equals lengths, they must be conjugate by Proposition 2.6.

Proof. We have that $|S| \geq\left|S_{5} S_{6}\right|$, so $\ell>1$. Let $u$ be the suffix of $S$ of length $|S|-\ell$. Since $\ell$ is repetitive, the factor $v$ of $S^{3}$ of length $\left|S^{2}\right|$ starting at position $\ell$ can be factorized as a product of minimal squares $Y_{1}^{2} \cdots Y_{m}^{2}$. We have that $\left|Y_{1}^{2}\right|>|u|$ because $\ell \in \mathcal{B}_{S}$. 
Next we consider how the situation changes if any of the words $S$ in $S^{3}$ is substituted with $L$. Substituting the first $S$ with $L$ does not affect the product as $\ell>1$. Suppose then that the second word $S$ is substituted with $L$. By applying Lemma 5.5 to the words $u$ and $S$ with $X=Y_{1}$, we see that the factor of length $\left|S^{2}\right|$ of $S L S$ starting at position $\ell$ can still be factorized as a product of minimal squares and that the square root of this factor coincides with the square root of $v$. Consider next what happens when the third word $S$ is substituted with $L$. Let

$$
r=\max \left\{i \in\{1, \ldots, m\}:\left|Y_{1}^{2} \cdots Y_{i}^{2}\right| \leq\left|S^{2}\right|-\ell\right\} .
$$

Set $\ell^{\prime}=\ell+\left|Y_{1}^{2} \cdots Y_{r}^{2}\right|-|S|$. Since $\ell$ is nicely repetitive, we have that $\ell^{\prime}<|S|$. By the maximality of $r$ and the definition of the set $\mathcal{B}_{S}$, we thus have that $\ell^{\prime} \in \mathcal{B}_{S}$. Applying Lemma 5.5 to the suffix of $S$ of length $|S|-\ell^{\prime}$ and $S$ with $X=Y_{r+1}$ we obtain, like above, that the product of minimal squares is affected but the square root is not. Substituting the second and third words $S$ with $L$ gives the same result: first proceed as above and substitute the second word $S$ and then make the second substitution like above but apply Lemma 5.5 for the word $L$ instead of $S$.

We have concluded that however we substitute the words $S$ in $S^{3}$, the square root of the factor of length $\left|S^{2}\right|$ beginning at position $\ell$ never changes. The word $w$ is obtained from the word $S^{\omega}$ by substituting some of the words $S$ with $L$. By the preceding, the prefix of $T^{\ell}(w)$ of length $\left|S^{2}\right|$ can be factorized as a product of minimal squares $X_{1}^{2} \cdots X_{n}^{2}$. Since $\ell$ is repetitive, the prefix of $T^{\ell+\left|S^{2}\right|}(w)$ of length $\left|S^{2}\right|$ can also be factorized as a product of some minimal squares (perhaps different) but the square root still equals $X_{1} \cdots X_{n}$. By repeating this observation we see that

$$
\sqrt{T^{\ell}(w)}=\left(X_{1} \cdots X_{n}\right)^{\omega} .
$$

By our choice of $S$ we have that $S \in\left\{\widetilde{s}_{k}, L\left(\widetilde{s}_{k}\right)\right\}$ where $\widetilde{s}_{k}$ is a reversed standard word of some slope $\alpha=[0 ; \mathfrak{a}+1, \mathfrak{b}+1, \ldots]$. Let $\beta=\left[0 ; b_{1}, b_{2}, \ldots\right]$ be a number such that $a_{i}=b_{i}$ for $1 \leq i \leq k$ and $b_{k+1} \geq 5$. Then by the definition of standard words $S^{5} \in \mathcal{L}(\beta)$. By the preceding, the prefix of $T^{\ell}\left(S^{5}\right)$ of length $\left|S^{4}\right|$ can be written as a product of minimal squares, and the square root of these minimal squares equals $\left(X_{1} \cdots X_{n}\right)^{2}$. Since the square root of a Sturmian word of slope $\beta$ is a Sturmian word of slope $\beta$, we have that $\left(X_{1} \cdots X_{n}\right)^{2} \in \mathcal{L}(\beta)$. As $\left|X_{1} \cdots X_{n}\right|=|S|$, it follows by Proposition 2.6 that $X_{1} \cdots X_{n}$ is conjugate to $S$. Since $S$ is primitive, so is $X_{1} \cdots X_{n}$, and hence the period $X_{1} \cdots X_{n}$ is minimal.

Lemma 8.11. Every seed solution $S$ has at least one nicely repetitive position $\ell$ such that $\ell \in \mathcal{B}_{S}$.

Proof. Suppose that $S=\widetilde{s}_{k, i}$ for some $k \geq 3$ and $0<i \leq a_{k}$. It is sufficient to show that $r=\left|\widetilde{s}_{k, i-1}\right|$ is a nicely repetitive position of $S$. If $r \notin \mathcal{B}_{S}$, then there exists $r^{\prime} \in \mathcal{B}_{S}$ such that $S\left[r, r^{\prime}-1\right] \in$ $\Pi(\mathfrak{a}, \mathfrak{b})$. Since the position $r$ is nicely repetitive, so must $r^{\prime}$ be. If $S=L\left(\widetilde{s}_{k, i}\right)$, then as $r>1$, an application of Lemma 5.5 shows that the conclusion holds also in this case.

Observe that the word $\widetilde{s}_{k, i-1}$ is both a prefix and a suffix of $S$. Using the fact that $\widetilde{s}_{k-2} \widetilde{s}_{k-3}=$ $L\left(\widetilde{s}_{k-3} \widetilde{s}_{k-2}\right)$ we obtain that

$$
\begin{aligned}
S^{3} & =\widetilde{s}_{k, i-1} \widetilde{s}_{k-1} \widetilde{s}_{k-2} \widetilde{s}_{k-1}^{i} \widetilde{s}_{k, i}=\widetilde{s}_{k, i-1} \cdot \widetilde{s}_{k-1} \widetilde{s}_{k-2} \widetilde{s}_{k-3} \widetilde{s}_{k-2}^{a_{k-1}-1} \cdot \widetilde{s}_{k, i-1} \widetilde{s}_{k, i} \\
& =\widetilde{s}_{k, i-1} \cdot \widetilde{s}_{k-1} L\left(\widetilde{s}_{k-1}\right) \cdot \widetilde{s}_{k, i-1}^{2} \widetilde{s}_{k-1} .
\end{aligned}
$$

By Lemma 5.6 the word $\widetilde{s}_{k-1} L\left(\widetilde{s}_{k-1}\right)$ is in $\Pi(\mathfrak{a}, \mathfrak{b})$. Since $\widetilde{s}_{k, i-1}$ is a solution to (8), we have that $\widetilde{s}_{k, i-1}^{2} \in \Pi(\mathfrak{a}, \mathfrak{b})$. Overall, the factor $\widetilde{s}_{k-1} L\left(\widetilde{s}_{k-1}\right) \widetilde{s}_{k, i-1}^{2}$ of $S^{3}$ of length $\left|S^{2}\right|$ starting at position $r$ is in $\Pi(\mathfrak{a}, \mathfrak{b})$. Thus the position $r$ of $S$ is repetitive.

Suppose for a contradiction that the suffix of $S$ of length $|S|-r$ is in $\Pi(\mathfrak{a}, \mathfrak{b})$, that is, $S=$ $\widetilde{s}_{k, i-1} X_{1}^{2} \cdots X_{n}^{2}$ for some minimal square roots $X_{j}$. It follows that $s_{k-1}=X_{1}^{2} \cdots X_{n}^{2}$. Since $s_{k-1}$ is a solution to (8), it follows that $s_{k-1}=\left(X_{1} \cdots X_{n}\right)^{2}$. This contradicts the primitivity of $s_{k-1}$. Similarly if the suffix of $S^{2}$ of length $\left|S^{2}\right|-r$ is in $\Pi(\mathfrak{a}, \mathfrak{b})$, then $\widetilde{s}_{k, i-1} \in \Pi(\mathfrak{a}, \mathfrak{b})$ contradicting the primitivity of $\widetilde{s}_{k, i-1}$. We conclude that the position $r$ is nicely repetitive. 
Lemma 8.10 and Lemma 8.11 now imply the following:

Corollary 8.12. There exist uncountably many linearly recurrent optimal squareful words having (purely) periodic square root.

Proof. We only need to show that there are uncountably many such words. Consider the words in $\Omega$ which can be written as a product of the words $S$ and $L$. Viewed over the binary alphabet $\{S, L\}$, these words form an infinite subshift $\bar{\Omega}$. Let us show that $\bar{\Omega}$ is minimal. Then the conclusion follows by well-known arguments from topology: a minimal subshift is always finite or uncountable and an aperiodic subshift cannot be finite (use the fact that a perfect set is always uncountable).

Let $\bar{w} \in \bar{\Omega}$ (we use the notation of the proof of Lemma 8.8). Let $\bar{u} \in \mathcal{L}(\bar{w})$ be a factor such that $|\bar{u}| \geq 6$. As $|u| \geq 6|S|$, every occurrence of $u$ in $\Gamma$ must synchronize to the factorization of $\Gamma$ as a product of $S$ and $L$. It follows that every return to $u$ in $\Gamma$ is a product of $S$ and $L$. Since the return time of $u$ is finite in $\Gamma$, the return time of the word $\bar{u}$ in $\bar{w}$ is also finite. Hence $\bar{\Omega}$ is minimal.

We also prove the following weaker result, which we need later.

Lemma 8.13. The position $|S|-\left|S_{6}\right|$ of $S$ is repetitive.

Proof. We prove first by induction that the prefix of the word $S_{6} \widetilde{s}_{k, \ell}^{2}$ of length $2\left|\widetilde{s}_{k, \ell}\right|-\left|S_{6}\right|$ is a product of minimal squares for $k \geq 2$ and $\ell$ such that $0<\ell \leq a_{k}$. Let us first establish the base cases.

Recall that $\widetilde{s}_{2}=0\left(10^{\mathfrak{a}}\right)^{\mathfrak{b}+1}$ and $\widetilde{s}_{3,1}=S_{6}$. We have that

$$
S_{6} \widetilde{S}_{2}^{2}=10^{\mathfrak{a}+1}\left(10^{\mathfrak{a}}\right)^{\mathfrak{b}+1}\left(0\left(10^{\mathfrak{a}}\right)^{\mathfrak{b}+1}\right)^{2}=S_{5}^{2} 10^{\mathfrak{a}+1}\left(10^{\mathfrak{a}}\right)^{\mathfrak{b}+1}=S_{5}^{2} S_{6} .
$$

In addition, for $0<\ell \leq a_{3}$, we have that

$$
S_{6} \widetilde{s}_{3, \ell}^{2}=S_{6} \widetilde{s}_{3,1} \widetilde{s}_{2}^{\ell-1} \widetilde{s}_{3, \ell}=S_{6}^{2} \widetilde{s}_{2}^{\ell-1} \widetilde{s}_{3, \ell}=S_{6}^{2} \widetilde{s}_{2}^{\ell-1} \widetilde{s}_{1} \widetilde{s}_{2}^{\ell} .
$$

The case $\ell=1$ is clear. So let us assume that $\ell>1$. We have that

$$
S_{6} \widetilde{S}_{3, \ell}^{2}=S_{6}^{2} \widetilde{S}_{2}^{\ell-1} \widetilde{S}_{1} \widetilde{S}_{2}^{\ell-2} \widetilde{S}_{0} \widetilde{S}_{1}^{\mathfrak{b}} S_{6}
$$

so it is sufficient to show that the word $\widetilde{s}_{2}^{\ell-1} \widetilde{s}_{1} \widetilde{s}_{2}^{\ell-2} \widetilde{s}_{0} \widetilde{s}_{1}^{\mathfrak{b}}$ is in $\Pi(\mathfrak{a}, \mathfrak{b})$.

Suppose first that $\ell-1$ is even. Then as $\widetilde{s}_{2}$ is a solution to (8), it is enough to show that $\widetilde{s}_{1} \widetilde{s}_{2}^{\ell-2} \widetilde{s}_{0} \widetilde{s}_{1}^{\mathfrak{b}} \in \Pi(\mathfrak{a}, \mathfrak{b})$. Since $\widetilde{s}_{1} \widetilde{s}_{2}=L\left(\widetilde{s}_{2}\right) \widetilde{s}_{1}$, we have that

$$
\widetilde{s}_{1} \widetilde{s}_{2}^{\ell-2} \widetilde{s}_{0} \widetilde{S}_{1}^{\mathfrak{b}}=L\left(\widetilde{s}_{2}\right)^{\ell-2} \widetilde{s}_{1} \widetilde{s}_{0} \widetilde{s}_{1}^{\mathfrak{b}} .
$$

Now $\widetilde{s}_{1} \widetilde{s}_{0} \widetilde{s}_{1}^{\mathfrak{b}}=L\left(\widetilde{s}_{2}\right)$. The word $L\left(\widetilde{s}_{2}\right)$ is a solution to (8), so the conclusion follows as $\ell-1$ is even.

Suppose next that $\ell-1$ is odd. We need to show that $\widetilde{s}_{2} \widetilde{S}_{1} \widetilde{S}_{2}^{\ell-2} \widetilde{s}_{0} \widetilde{s}_{1}^{\mathfrak{b}} \in \Pi(\mathfrak{a}, \mathfrak{b})$. Using the facts $\widetilde{s}_{1} \widetilde{s}_{2}=L\left(\widetilde{s}_{2}\right) \widetilde{s}_{1}$ and $\widetilde{s}_{1} \widetilde{s}_{0} \widetilde{s}_{1}^{\mathfrak{b}}=L\left(\widetilde{s}_{2}\right)$ we obtain that

$$
\widetilde{s}_{2} \widetilde{s}_{1} \widetilde{s}_{2}^{\ell-2} \widetilde{s}_{0} \widetilde{s}_{1}^{\mathfrak{b}}=\widetilde{s}_{2} L\left(\widetilde{s}_{2}\right)^{\ell-1} .
$$

By Lemma 5.6 the word $\widetilde{s}_{2} L\left(\widetilde{s}_{2}\right)$ is a product of minimal squares. Since $\ell-1$ is odd and $L\left(\widetilde{s}_{2}\right)$ is a solution to (8), the conclusion follows.

We have established the base cases. Now for $k \geq 4$ and $0<\ell \leq a_{k}$, we have that

$$
S_{6} \widetilde{s}_{k, \ell}^{2}=S_{6}\left(\widetilde{s}_{k-2} \widetilde{s}_{k-1}^{\ell}\right)^{2} .
$$

By induction $S_{6} \widetilde{s}_{k-2}=X_{1}^{2} \cdots X_{n}^{2} S_{6}$ and $S_{6} \widetilde{s}_{k-1}=Y_{1}^{2} \cdots Y_{m}^{2} S_{6}$ for some minimal square roots $X_{1}, \ldots, X_{n}, Y_{1}, \ldots, Y_{m}$. Therefore

$$
S_{6} \widetilde{s}_{k, \ell}^{2}=\left(X_{1}^{2} \cdots X_{n}^{2}\left(Y_{1}^{2} \cdots Y_{m}^{2}\right)^{\ell}\right)^{2} S_{6} .
$$


We have thus proved that the prefix of the word $S_{6} \widetilde{s}_{k, \ell}^{2}$ of length $2\left|\widetilde{s}_{k, \ell}\right|-\left|S_{6}\right|$ is a product of minimal squares for $k \geq 2$ and $\ell$ such that $0<\ell \leq a_{k}$.

Now if $S=\widetilde{s}_{k, \ell}$ for some $k \geq 2$ and $\ell$ such that $0<\ell \leq a_{k}$, then the claim is clear by the above. Suppose that $S=L\left(\widetilde{s}_{k, \ell}\right)$. Now if $S_{6} \widetilde{s}_{k, \ell} \notin \Pi(\mathfrak{a}, \mathfrak{b})$, then two applications of Lemma 5.5 (first with $u=S_{6}, v=S^{2}$ and then with $u=S_{6} L, v=S$ ) show that the claim holds. Assume that $S_{6} \widetilde{s}_{k, \ell} \in \Pi(\mathfrak{a}, \mathfrak{b})$. Since the prefix of $S_{6} \widetilde{s}_{k, \ell}$ of length $2\left|\widetilde{s}_{k, \ell}\right|-\left|S_{6}\right|$ is in $\Pi(\mathfrak{a}, \mathfrak{b})$, this means that the prefix of $\widetilde{s}_{k, \ell}$ of length $\left|\widetilde{s}_{k, \ell}\right|-\left|S_{6}\right|$ is in $\Pi(\mathfrak{a}, \mathfrak{b})$. It is sufficient to show that the prefixes of $\widetilde{s}_{k, \ell}$ and $L\left(\widetilde{s}_{k, \ell}\right)$ of length $2\left|\widetilde{s}_{2}\right|$ are in $\Pi(\mathfrak{a}, \mathfrak{b})$. Since $\widetilde{s}_{1} \widetilde{s}_{2}=L\left(\widetilde{s}_{2}\right) \widetilde{s}_{1}$, the word $\widetilde{s}_{4,1}=\widetilde{s}_{2} \widetilde{s}_{3}$ has $\widetilde{s}_{2} L\left(\widetilde{s}_{2}\right)$ as a prefix. If $a_{3}>1$, then the word $\widetilde{s}_{3}=\widetilde{s}_{1} \widetilde{s}_{2}^{a_{3}}$ has $L\left(\widetilde{s}_{2}\right) \widetilde{s}_{2}$ as a prefix. Finally if $a_{3}=1$, then the word $\widetilde{s}_{5,1}=\widetilde{s}_{3} \widetilde{s}_{4}=\widetilde{s}_{1} \widetilde{s}_{2} \widetilde{S}_{4}$ has $L\left(\widetilde{s}_{2}\right)^{2}$ as a prefix. Lemma 5.6 shows that $\widetilde{s}_{2} L\left(\widetilde{s}_{2}\right), L\left(\widetilde{s}_{2}\right) \widetilde{s}_{2}$, and $L\left(\widetilde{s}_{2}\right)^{2}$ are all in $\Pi(a, b)$. The conclusion follows.

There is no clear pattern for other positions in $\mathcal{B}_{S}$; it depends on the word $S$ if a position in $\mathcal{B}_{S}$ is repetitive or not. The position $|S|-\left|S_{6}\right|$ is not always nicely repetitive. Suppose that $\mathfrak{a}=1$, $\mathfrak{b}=0$, and $S=\widetilde{s}_{3,3}=10(010)^{3}$. Then the factor beginning at position $|S|-\left|S_{6}\right|=6$ of $S^{3}$ of length $\left|S^{2}\right|$ is a product of minimal squares: $(10010)^{2} \cdot(010)^{2} \cdot(100)^{2}$. As $\left|(10010)^{2} \cdot(010)^{2}\right|=$ $16=\left|S^{2}\right|-6$, the position 6 is not nicely repetitive.

Since none of the minimal squares can be a proper prefix of another minimal square, it is easy to factorize words as products of minimal squares from left to right. Next we consider what happens if we start to backtrack from a given position to the left.

Lemma 8.14 (Backtracking Lemma). Let $X, Y_{1}, \cdots Y_{n}$ be minimal square roots. Let w be a word having both of the words $X^{2}$ and $Y_{1}^{2} \cdots Y_{n}^{2}$ as suffixes. If $|X|>\left|Y_{n}\right|$, then $|X|>\left|Y_{1} \cdots Y_{n}\right|$ and the word $Y_{1} \cdots Y_{n}$ is a suffix of $X$.

Proof. Suppose that $|X|>\left|Y_{n}\right|$. We may assume that $n$ is as large as possible. We prove the lemma by considering different options for the word $X$.

Clearly we cannot have that $X=S_{1}$. Let $X=S_{4}$. Now $X^{2}$ can have a proper minimal square suffix only if $\mathfrak{a}>1$. If $\mathfrak{a}$ is even, then we must have that

$$
X^{2}=10^{\mathfrak{a}} 1\left(S_{1}^{2}\right)^{\mathfrak{a} / 2} \text { and } Y_{n-\mathfrak{a} / 2+1}=\ldots=Y_{n}=S_{1} .
$$

The suffix $\left(S_{1}\right)^{\mathfrak{a} / 2}$ of $w$ cannot be preceded by $S_{2}^{2}$ as otherwise $w$ would have $S_{2} S_{1}^{\mathfrak{a}}=010^{2 \mathfrak{a}-1}$ as a suffix; this is not possible as $2 \mathfrak{a}-1>\mathfrak{a}$. Therefore there is no choice for $Y_{n-\mathfrak{a} / 2}$. Thus $\left|Y_{1}^{2} \cdots Y_{n}^{2}\right|<\left|X^{2}\right|$ and $Y_{1} \cdots Y_{n}$ is a suffix of $X$. If $\mathfrak{a}$ is odd, then similarly

$$
X^{2}=10^{\mathfrak{a}} 10\left(S_{1}^{2}\right)^{(\mathfrak{a}-1) / 2} \text { and } Y_{n-(\mathfrak{a}-1) / 2+1}=\ldots=Y_{n}=S_{1} .
$$

Again there is no choice for $Y_{n-(\mathfrak{a}-1) / 2}$, and the conclusion holds. Similar considerations show that the conclusion holds if $X \in\left\{S_{2}, S_{3}\right\}$.

Let then $X=S_{5}$. It is obvious that now $Y_{n} \in\left\{S_{1}, S_{3}, S_{4}\right\}$. If $Y_{n}=S_{1}$ or $\mathfrak{b}=0$, then like above $Y_{1}=\ldots=Y_{n}=S_{1}$ and $Y_{1} \cdots Y_{n}$ is a suffix of $X$. We may thus suppose that $\mathfrak{b}>0$. Say $Y_{n}=S_{3}$. Then we must have $\mathfrak{b}=1$ and $X^{2}=10^{\mathfrak{a}+1} 10^{\mathfrak{a}-1} Y_{n}^{2}$. Like above, the remaining minimal square roots $Y_{i}$ with $i<n$ must equal to $S_{1}$ and there must be $\lfloor(\mathfrak{a}-1) / 2\rfloor$ of them. Since there is no further choice, the conclusion holds as clearly $Y_{1} \cdots Y_{n}$ is a suffix of $X$. Suppose then that $\mathfrak{b}>1$. The next case is $Y_{n}=S_{4}$. Assume first that $\mathfrak{b}$ is even. Then it is straightforward to see that necessarily

$$
Y_{n-\mathfrak{b} / 2+1}=\ldots=Y_{n}=S_{4} \text { and } X^{2}=10^{\mathfrak{a}+1}\left(10^{\mathfrak{a}}\right)^{\mathfrak{b}} 10^{\mathfrak{a}+1}\left(S_{4}^{2}\right)^{\mathfrak{b} / 2} .
$$

Thus $Y_{n-\mathfrak{b} / 2}=S_{1}$ and, further, it must be that

$$
Y_{n-\mathfrak{b}}=\ldots=Y_{n-\mathfrak{b} / 2-1}=S_{2} \text { and } X^{2}=10^{\mathfrak{a}+1} 10^{\mathfrak{a}-1}\left(S_{2}^{2}\right)^{\mathfrak{b} / 2} S_{1}^{2}\left(S_{4}^{2}\right)^{\mathfrak{b} / 2} .
$$


Like before, the remaining minimal squares $Y_{i}$ with $i<n-\mathfrak{b}$ must equal to $S_{1}$ and there must be $\lfloor(\mathfrak{a}-1) / 2\rfloor$ of them. Therefore

$$
Y_{1} \cdots Y_{n}=S_{1}^{\lfloor(\mathfrak{a}-1) / 2\rfloor} S_{2}^{\mathfrak{b} / 2} S_{1} S_{4}^{\mathfrak{b} / 2}=0^{\lfloor(\mathfrak{a}-1) / 2\rfloor+1}\left(10^{\mathfrak{a}}\right)^{\mathfrak{b}}
$$

is a suffix of $X$, so the conclusion holds. If $\mathfrak{b}$ is odd, then in a similar fashion

$$
X^{2}=10^{\mathfrak{a}+1} 10^{\mathfrak{a}-1}\left(S_{2}^{2}\right)^{(\mathfrak{b}-1) / 2} S_{3}^{2}\left(S_{4}\right)^{(\mathfrak{b}-1) / 2},
$$

so $Y_{n-(\mathfrak{b}-1) / 2}=S_{3}$ and

$$
Y_{n-(\mathfrak{b}-1) / 2+1}=\ldots=Y_{n}=S_{4} \text { and } Y_{n-\mathfrak{b}+1}=\ldots=Y_{n-(\mathfrak{b}-1) / 2-1}=S_{2} .
$$

Again, the final $\lfloor(\mathfrak{a}-1) / 2\rfloor$ minimal square roots must equal $S_{1}$. Since

$$
Y_{1} \cdots Y_{n}=S_{1}^{\lfloor(\mathfrak{a}-1) / 2\rfloor} S_{2}^{(\mathfrak{b}-1) / 2} S_{3} S_{4}^{(\mathfrak{b}-1) / 2}=0^{\lfloor(\mathfrak{a}-1) / 2\rfloor+1}\left(10^{\mathfrak{a}}\right)^{\mathfrak{b}}
$$

is a suffix of $X$, the conclusion holds.

If $X=S_{6}$, then it is clear that $Y_{n} \neq S_{5}$. The conclusion follows as in the case $X=S_{5}$.

The next lemma is useful in the proof of Theorem 8.7.

Lemma 8.15. Let $w$ be an infinite product of the words $S$ and $L$ and $\ell_{1}, \ell_{2}, \ell_{3}$ be positions of $w$ such that $\ell_{1}<\ell_{2}<\ell_{3}$. Let $r$ be the largest integer such that $\ell_{1} \geq r|S|$. If

- $w\left[\ell_{1}, \ell_{3}-1\right], w\left[\ell_{2}, \ell_{3}-1\right] \in \Pi(\mathfrak{a}, \mathfrak{b})$,

- $\ell_{1}-r|S| \in \mathcal{B}_{S}$, and

- $\ell_{2} \leq(r+1)|S|$,

then for all $u \in \Pi(\mathfrak{a}, \mathfrak{b})$ such that $u w\left[\ell_{2}, \ell_{3}-1\right]$ is a suffix of $w\left[0, l_{3}-1\right]$ we have that $\left|u w\left[\ell_{2}, \ell_{3}-1\right]\right|<$ $\left|w\left[\ell_{1}, \ell_{3}-1\right]\right|$.

Proof. Let $v=w\left[\ell_{1}, \ell_{3}-1\right]$ and $u=w\left[\ell_{2}, \ell_{3}-1\right]$. Since $v, u \in \Pi(\mathfrak{a}, \mathfrak{b})$, we may write $v=X_{1}^{2} \cdots X_{n}^{2}$ and $u=Y_{1}^{2} \cdots Y_{m}^{2}$ for some minimal square roots $X_{i}$ and $Y_{i}$. If $n \geq m$ and $X_{n-m+i}=Y_{i}$ for all $i \in\{1, \ldots, m\}$, then as $|v|>|u|$, we must have that $n>m$. This means that the prefix $X_{1}^{2}$ of $v$ ends before the position $\ell_{2}$, that is, $\ell_{1}+\left|X_{1}^{2}\right|<\ell_{2} \leq(r+1)|S|$. This contradicts the fact that $\ell_{1}-r|S| \in \mathcal{B}_{S}$. Therefore as $|v|>|u|$, we we conclude that there exists maximal $j \in\{1, \ldots, m\}$ such that $X_{n-m+j} \neq Y_{j}$. If $\left|Y_{j}\right|>\left|X_{n-m+j}\right|$, then by the Backtracking Lemma we have that $\left|X_{1}^{2} \cdots X_{n-m+j}^{2}\right|<\left|Y_{j}^{2}\right|$. This is not possible as $|v|>|u|$. Therefore $\left|Y_{j}\right|<\left|X_{n-m+j}\right|$. Let $z \in$ $\Pi(\mathfrak{a}, \mathfrak{b})$ be such that $z u$ is a suffix of $w\left[0, l_{3}-1\right]$. Write $z=Z_{1}^{2} \cdots Z_{t}^{2}$ for minimal square roots $Z_{i}$. Applying the Backtracking Lemma to the words $X_{n-m+j}^{2}$ and $Z_{1}^{2} \cdots Z_{t}^{2} Y_{1}^{2} \cdots Y_{j}^{2}$ yields that $\left|Z_{1}^{2} \cdots Z_{t}^{2} Y_{1}^{2} \cdots Y_{j}^{2}\right|<\left|X_{n-m+j}^{2}\right|$. It follows that $|z u|<|v|$.

Finally we can give a proof of Theorem 8.7.

Proof of Theorem 8.7. Let $w \in \Omega$. Since $\Gamma$ is uniformly recurrent and a product of the words $S$ and $L$, there exists a word $w^{\prime} \in \Omega$ such that $w^{\prime}$ is a product of $S$ and $L$ and $w=T^{\ell}\left(w^{\prime}\right)$ for some $\ell$ such that $0 \leq \ell<|S|$ (recall that a product of $S$ and $L$ occurring in $\Gamma$ having length at least $6|S|$ must synchronize to the factorization of $\Gamma$ as a product of $S$ and $L$ ). If $\ell=0$, then the conclusion holds by Lemma 8.8, so we can assume that $\ell>0$. Write $w$ as a product of minimal squares: $w=X_{1}^{2} X_{2}^{2} \cdots$. Let

$$
r_{1}=\max \left\{\{0\} \cup\left\{i \in\{1,2, \ldots\}:\left|X_{1}^{2} \cdots X_{i}^{2}\right| \leq|S|-\ell\right\}\right\} .
$$


If $r_{1}>0$, then set $\ell_{1}=\ell+\left|X_{1}^{2} \cdots X_{r_{1}}^{2}\right|$. If $r_{1}=0$, then we set $\ell_{1}=\ell$. By the maximality of $r_{1}$ and by the definition of the set $\mathcal{B}_{S}$, it follows that $\ell_{1} \in \mathcal{B}_{S} \cup\{|S|\}$ (indeed, the word $L$ also has $S_{6}$ as a suffix). See Figure 5.

To aid comprehension we have separated different parts of the proof as distinct claims with their own proofs. Any new definitions and assumptions given in one of the subproofs are valid only up to the end of the subproof.

Claim 8.15.1. If $\ell_{1}=|S|$, then $\sqrt{w} \in \Omega$.

Proof. Suppose that $\ell_{1}=|S|$. By the definition of the number $r_{1}$, we have that $r_{1}>0$ and the word $T^{|S|-\ell}(w)=T^{|S|}\left(w^{\prime}\right)=X_{r_{1}+1}^{2} X_{r_{2}+2}^{2} \cdots$ is a product of the words $S$ and $L$. Now $z w^{\prime} \in \Omega$ where $z \in\{S, L\}$. Since $z w^{\prime}$ is a product of $S$ and $L$, by Lemma $8.8 \sqrt{z w^{\prime}} \in \Omega$. By the choice of $S$ as a solution to (8) and by Lemma 5.6, the first $\left|S^{2}\right|$ letters of $z w^{\prime}$ can be written as a product of minimal squares. Hence $z w^{\prime}=Y_{1}^{2} \cdots Y_{n}^{2} X_{r_{1}+1}^{2} X_{r_{1}+2}^{2} \cdots$ for some minimal square roots $Y_{1}, \ldots, Y_{n}$. By the Backtracking Lemma, we have that $X_{1} \cdots X_{r_{1}}$ is a suffix of $Y_{1} \cdots Y_{n}$. Thus the word $\sqrt{w}=$ $X_{1} \cdots X_{r_{1}} X_{r_{1}+1} \cdots$ is a suffix of the word $\sqrt{z w^{\prime}}=Y_{1} \cdots Y_{n} X_{r_{1}} X_{r_{1}+1} \cdots$. Therefore $\mathcal{L}(\sqrt{w}) \subseteq$ $\mathcal{L}\left(\sqrt{z w^{\prime}}\right)=\mathcal{L}(\Gamma)$, so $\sqrt{w} \in \Omega$.

We assume that $\ell_{1} \in \mathcal{B}_{S}$. Now either the position $\ell_{1}$ of $S$ is nicely repetitive or it is not.

Claim 8.15.2. If $\ell_{1}$ is a nicely repetitive position of $S$, then $\sqrt{w}$ is periodic with minimal period conjugate to $S$.

Proof. By Lemma 8.10 the word $\sqrt{T^{\ell_{1}\left(w^{\prime}\right)}}$ is periodic with minimal period $z$ conjugate to $S$. If $\ell_{1}=\ell$, then there is nothing more to prove, so assume that $\ell_{1} \neq \ell$. There exists $u, v \in\{S, L\}$ such that $u v w^{\prime} \in \Omega$. Since $\ell_{1}$ is a nicely repetitive position of $S$, the prefix of $T^{\ell_{1}}\left(u v w^{\prime}\right)$ of length $\left|S^{2}\right|$ is a product of minimal squares and its square root equals $z$ by Lemma 8.10. Since the factor $w^{\prime}\left[\ell_{,} \ell_{1}-\right.$ $1]$ is also a product of minimal squares, the Backtracking Lemma implies that $\sqrt{w^{\prime}\left[\ell, \ell_{1}-1\right]}$ is a suffix of $z$. Now $\sqrt{w}=\sqrt{w^{\prime}\left[\ell, \ell_{1}-1\right]} \sqrt{T^{\ell_{1}}\left(w^{\prime}\right)}$, so $\sqrt{w}$ is periodic with minimal period conjugate to $S$.

If the position $\ell_{1}$ of $S$ is not nicely repetitive, then either it is not repetitive or it is repetitive but not nicely repetitive.

Claim 8.15.3. If $\ell_{1}$ is repetitive but not nicely repetitive position of $S$, then $\sqrt{w} \in \Omega$.

Proof. Suppose that $\ell_{1}$ is a repetitive but not a nicely repetitive position of $S$. This means that either $S^{3}\left[\ell_{1},|S|-1\right] \in \Pi(\mathfrak{a}, \mathfrak{b})$ or $S^{3}\left[\ell_{1},\left|S^{2}\right|-1\right] \in \Pi(\mathfrak{a}, \mathfrak{b})$ (they both cannot be in $\Pi(\mathfrak{a}, \mathfrak{b})$ as this would imply that $S$ is not primitive). Thus either $w^{\prime}\left[\ell_{1},|S|-1\right] \in \Pi(\mathfrak{a}, \mathfrak{b})$ or $w^{\prime}\left[\ell_{1},\left|S^{2}\right|-1\right] \in$ $\Pi(\mathfrak{a}, \mathfrak{b})$ (in the latter case Lemma 5.5 ensures that $w^{\prime}\left[\ell_{1},\left|S^{2}\right|-1\right] \in \Pi(\mathfrak{a}, \mathfrak{b})$ ). The former case is, however, not possible as it would contradict the maximality of $r_{1}$. Thus only the latter option is possible. Since $w^{\prime}$ is a product of the words $S$ and $L$, the prefix $w^{\prime}\left[0,\left|S^{2}\right|-1\right]$ of $w^{\prime}$ is a product of minimal squares. Since $w^{\prime}\left[\ell, \ell_{1}-1\right], w^{\prime}\left[\ell_{1},\left|S^{2}\right|-1\right] \in \Pi(\mathfrak{a}, \mathfrak{b})$, the Backtracking Lemma implies that $\sqrt{w^{\prime}\left[\ell,\left|S^{2}\right|-1\right]}$ is a suffix of $\sqrt{w^{\prime}\left[0,\left|S^{2}\right|-1\right]}$. Thus $\sqrt{w}$ is a suffix of $\sqrt{w^{\prime}}$. As $\sqrt{w^{\prime}} \in \Omega$ by Lemma 8.8, we conclude that $\sqrt{w} \in \Omega$.

Now we may suppose that $\ell_{1}$ is not a repetitive position of $S$. We let

$$
\begin{aligned}
& r_{2}=\max \left\{i \in\left\{r_{1}+1, r_{1}+2, \ldots\right\}:\left|X_{1}^{2} \cdots X_{i}^{2}\right| \leq\left|S^{2}\right|-\ell\right\}, \\
& r_{3}=\max \left\{i \in\left\{r_{2}+1, r_{2}+2, \ldots\right\}:\left|X_{1}^{2} \cdots X_{i}^{2}\right| \leq\left|S^{3}\right|-\ell\right\}, \text { and } \\
& r_{4}=\max \left\{i \in\left\{r_{3}+1, r_{3}+2, \ldots\right\}:\left|X_{1}^{2} \cdots X_{i}^{2}\right| \leq\left|S^{4}\right|-\ell\right\} .
\end{aligned}
$$


The numbers $r_{2}, r_{3}$, and $r_{4}$ are well-defined as the words $S$ and $L$ are not minimal squares. We set

$$
\begin{aligned}
& \ell_{2}=\ell_{1}+\left|X_{r_{1}+1}^{2} \cdots X_{r_{2}}^{2}\right|, \\
& \ell_{3}=\ell_{2}+\left|X_{r_{2}+1}^{2} \cdots X_{r_{3}}^{2}\right|, \text { and } \\
& \ell_{4}=\ell_{3}+\left|X_{r_{3}+1}^{2} \cdots X_{r_{4}}^{2}\right| .
\end{aligned}
$$

Intuitively, the positions $\ell_{1}, \ell_{2}, \ell_{3}$, and $\ell_{4}$ are the successive positions of $w$ which are closest from the left to the boundaries of the words $S$ and $L$ in the factorization of $w^{\prime}$ as a product of the words $S$ and $L$ such that the prefix up to the position is a product of minimal squares; see Figure 5. Let $g_{1}=\ell_{1}, g_{2}=\ell_{2}-|S|, g_{3}=\ell_{3}-\left|S^{2}\right|$, and $g_{4}=\ell_{4}-\left|S^{3}\right|$. It is clear by the definitions that $g_{i} \in B \cup\{|S|\}$ for all $i \in\{1,2,3,4\}$.

Claim 8.15.4. We have that $g_{1}, g_{3} \neq|S|$. If $g_{2}$ or $g_{4}$ equals $|S|$, then $\sqrt{w} \in \Omega$.

Proof. By our assumption that $\ell_{1} \in \mathcal{B}_{S}$, we have that $g_{1} \neq|S|$. If $g_{2}=|S|$, then the factor $w^{\prime}\left[\ell_{1},\left|S^{2}\right|-1\right]$ would be a product of minimal squares. This case was already considered in Claim 8.15.3 where we concluded that $\sqrt{w} \in \Omega$.

Suppose that $g_{3}=|S|$. Consider the positions $\ell_{1}$ and $|S|$ of $w^{\prime}$. Both of the factors $u=$ $w^{\prime}\left[\ell_{1}, \ell_{3}-1\right]$ and $v=w^{\prime}\left[|S|, \ell_{3}-1\right]=w^{\prime}\left[\ell_{3}-\left|S^{2}\right|, \ell_{3}-1\right]$ are in $\Pi(\mathfrak{a}, \mathfrak{b})$. Now $z w^{\prime} \in \Omega$ for some $z \in\{S, L\}$. Since $S S, S L, L S, L L \in \Pi(\mathfrak{a}, \mathfrak{b})$, the prefix of $z w^{\prime}$ of length $\left|S^{2}\right|$ is in $\Pi(\mathfrak{a}, \mathfrak{b})$. Lemma 8.15 applied to the word $\left(z w^{\prime}\right)\left[0,|S|+l_{3}-1\right]$ implies that $\left|\left(z w^{\prime}\right)\left[0,|S|+l_{3}-1\right]\right|<|u|<\left|S^{3}\right|$ which is nonsense. Therefore $g_{3} \neq|S|$.

Assume then that $g_{4}=|S|$. Suppose for a contradiction that $g_{2} \neq g_{4}$. Both of the factors $u^{\prime}=w^{\prime}\left[\ell_{2}, \ell_{4}-1\right]$ and $v^{\prime}=w^{\prime}\left[|S|^{2}, \ell_{4}-1\right]=w^{\prime}\left[\ell_{4}-\left|S^{2}\right|, \ell_{4}-1\right]$ are in $\Pi(\mathfrak{a}, \mathfrak{b})$. Since $g_{2} \neq g_{4}$, also $\ell_{2} \neq\left|S^{2}\right|$. Thus by the definition of $\ell_{2}$, we have that $\ell_{2}<\left|S_{2}\right|$. Lemma 8.15 applied to the word $w^{\prime}\left[0, \ell_{4}-1\right]$ shows that $\left|w^{\prime}\left[0, l_{4}-1\right]\right|<\left|u^{\prime}\right|<\left|S^{3}\right|$ which is absurd. This contradiction shows that $g_{2}=g_{4}=|S|$, so $\sqrt{w} \in \Omega$.

We may now assume that $g_{i} \in \mathcal{B}_{S}$ for all $i \in\{1,2,3,4\}$.

Claim 8.15.5. The position $g_{2}$ of $S$ is nicely repetitive.

Proof. Assume on the contrary that neither of the positions $g_{2}$ and $g_{3}$ is a repetitive position of $S$. First note that as $g_{1}$ is not repetitive, we have that $g_{3} \neq g_{1}$. Similarly $g_{2} \neq g_{4}$. If $g_{1}=g_{2}$, then it follows from Lemma 5.5 and the definitions of the positions $l_{2}$ and $l_{3}$ that $g_{2}=g_{3}$; a contradiction. Hence $g_{1} \neq g_{2}$. Similarly $g_{2} \neq g_{3}$ as otherwise the position $g_{2}$ would be repetitive. Finally, $g_{3} \neq g_{4}$ because $g_{3}$ is not repetitive. We have two cases: either $g_{1}=g_{4}$ or $g_{1} \neq g_{4}$.

Assume that $g_{4} \neq g_{1}$. By Lemma 8.13 the position $|S|-\left|S_{6}\right|$ of $S$ is repetitive, so $g_{1}, g_{2}, g_{3} \in$ $\mathcal{B}_{S} \backslash\left\{|S|-\left|S_{6}\right|\right\}=\left\{|S|-\left|S_{1}\right|,|S|-\left|S_{3}\right|,|S|-\left|S_{4}\right|\right\}$. Since all of the positions $g_{1}, g_{2}$, and $g_{3}$ are distinct, the only option is that $g_{4}=|S|-\left|S_{6}\right|$. Since the position $|S|-\left|S_{6}\right|$ is repetitive, by Lemma 5.5 the factor $u=w^{\prime}\left[\ell_{4}-\left|S^{2}\right|, \ell_{4}-1\right]$ is in $\Pi(\mathfrak{a}, \mathfrak{b})$. By the definition of the positions $\ell_{2}$, $\ell_{3}$, and $\ell_{4}$ also $v=w^{\prime}\left[\ell_{2}, \ell_{4}-1\right] \in \Pi(\mathfrak{a}, \mathfrak{b})$. Since $g_{2} \neq g_{4}$, also $\ell_{2} \neq \ell_{4}-\left|S^{2}\right|$. Since $|S|-\left|S_{6}\right|$ is the smallest element of the set $\mathcal{B}_{S}$, we have that $\ell_{2}>\ell_{4}-\left|S^{2}\right|$. As $w\left[l_{1}, l_{2}-1\right] \in \Pi(\mathfrak{a}, \mathfrak{b})$, we obtain by Lemma 8.15 that $\left|w\left[l_{1}, l_{4}-1\right]\right|<|u|=\left|S^{2}\right|$. This is a contradiction.

Hence we have that $g_{1}=g_{4}$. Since the factor $w\left[\ell_{1}, \ell_{2}-1\right]$ is a product of minimal squares, the number $c_{1}=\ell_{2}-\ell_{1}$ is even. Similarly the numbers $c_{2}=\ell_{3}-\ell_{2}$ and $c_{3}=\ell_{4}-\ell_{3}$ are even. Thus the number $c_{1}+c_{2}+c_{3}=3|S|$ is even, so $|S|$ is even. It follows that the numbers $d_{1}=g_{2}-g_{1}$, $d_{2}=g_{3}-g_{2}$, and $d_{3}=g_{4}-g_{3}=g_{1}-g_{3}$ are even. However, exactly two of the numbers $\left|S_{1}\right|$, $\left|S_{3}\right|$, and $\left|S_{4}\right|$ have odd length. Hence exactly two of the numbers $g_{1}, g_{2}$, and $g_{3}$ are odd. Thus it is not possible that all of the numbers $d_{1}, d_{2}$, and $d_{3}$ are even. This is a contradiction.

The previous contradiction shows that either of the positions $g_{2}$ and $g_{3}$ is a repetitive position of $S$. Suppose for a contradiction that $g_{3}$ is repetitive. We have that $w^{\prime}\left[\ell_{1}, \ell_{3}-1\right] \in \Pi(\mathfrak{a}, \mathfrak{b})$ and $w^{\prime}\left[\ell_{3}-\left|S^{2}\right|, \ell_{3}-1\right] \in \Pi(\mathfrak{a}, \mathfrak{b})$. Similar to the second paragraph of this subproof, using 


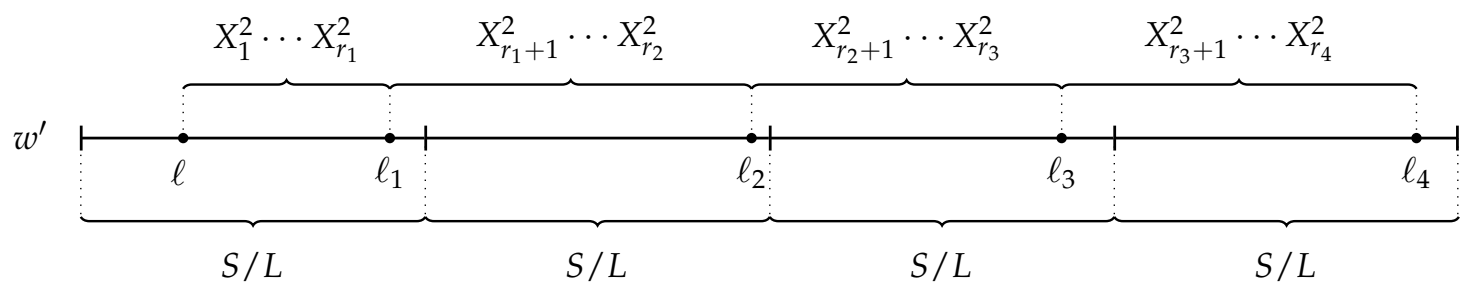

Figure 5: The positions $\ell_{,} \ell_{1}, \ell_{2}, \ell_{3}$, and $\ell_{4}$ of $w^{\prime}$ and the minimal squares between the positions.

Lemma 8.15 we obtain a contradiction unless $g_{1}=g_{3}$. Even this conclusion is contradictory as $g_{1}$ is not repetitive. Therefore $g_{3}$ can not be repetitive, so $g_{2}$ is a repetitive position of $S$. Now if $g_{2}$ would not be nicely repetitive, we would have by the maximality of $r_{2}$ that $w^{\prime}\left[\ell_{2},\left|S^{3}\right|-1\right] \in \Pi(\mathfrak{a}, \mathfrak{b})$, that is, $g_{3}=|S|$. However, since $g_{3} \in \mathcal{B}_{S}$, we have that $g_{2}$ is a nicely repetitive position of $S$.

We are now in the final stage of the proof. We will show that $\sqrt{w}$ is periodic with minimal period conjugate to $|S|$.

We can now argue as in the proof of Claim 8.15.2. Since $g_{2}$ is a nicely repetitive position of $S$, by Lemma 8.10 the word $\sqrt{T^{\ell_{2}}\left(w^{\prime}\right)}$ is periodic with minimal period $z$ conjugate to $S$. We have that $u w^{\prime} \in \Omega$ for some $u \in\{S, L\}$. Since $g_{2}$ is a nicely repetitive position of $S$, the prefix of $T^{g_{2}}\left(u w^{\prime}\right)$ of length $\left|S^{2}\right|$ is a product of minimal squares and its square root equals $z$ by Lemma 8.10. Since $w^{\prime}\left[\ell, \ell_{2}-1\right] \in \Pi(\mathfrak{a}, \mathfrak{b})$, the Backtracking Lemma implies that $\sqrt{w^{\prime}\left[\ell, \ell_{2}-1\right]}$ is a suffix of $z$. Now $\sqrt{w}=\sqrt{w^{\prime}\left[\ell, \ell_{2}-1\right]} \sqrt{T^{\ell_{2}\left(w^{\prime}\right)}}$, so $\sqrt{w}$ is periodic with minimal period conjugate to $S$.

By Lemma 8.11 the word $S$ always has at least one nicely repetitive position. It therefore follows that there exists a word in $\Omega$ having periodic square root.

\section{Remarks on Generalizations}

It is natural to think that the square root map could be generalized to obtain a cube root map and, further, a $k^{\text {th }}$ root map. However, in [16, Theorem 5.3.] Saari proves the following reformulation of a result of Mignosi, Restivo, and Salemi.

Proposition 9.1. If $w$ is an everywhere $\alpha$-repetitive word with $\alpha \geq \phi+1$, where $\phi$ is the golden mean, then $w$ is ultimately periodic.

Generalizing the square root map to a cube root map would require everywhere 3-repetitive words. By the above such words must be ultimately periodic, so we expect that this direction of research would not be fruitful.

Another way to generalize the square root map is to use abelian powers instead of ordinary powers. For abelian powers a result like Proposition 9.1 does not exist. For instance, by [15, Theorem 1.9.] every position in a Sturmian word begins with an abelian $k^{\text {th }}$ power for all $k \geq$ 2. Abelian square root can be defined for e.g. optimal squareful words as we will see shortly. However, abelian cubes in Sturmian words do not work. Consider again the Fibonacci word $f$. The minimal abelian cube prefix of $T(f)$ is $10 \cdot 01 \cdot 01$. This abelian cube is followed by the factor 00 , so the root of the next abelian cube must begin with 00 . Hence if we define the abelian cube root of $T(f)$ to be the product of the roots of the abelian cubes, the resulting word begins with 1000 which is not a factor of $f$. Thus by defining an abelian cube root map in this way, we lose the main property that the mapping preserves the languages of Sturmian words.

In [17] Saari also considers optimal abelian squareful words. Optimal abelian squareful words are defined by replacing minimal squares with minimal abelian squares in the definition of optimal squareful words. Let $w=X_{1} X_{1}^{\prime} X_{2} X_{2}^{\prime} \cdots$ be a product of minimal abelian squares $X_{i} X_{i}^{\prime}$. 
We define its abelian square root as the word $\sqrt[a b]{w}=X_{1} X_{2} \cdots$. It follows from [17, Theorem 18] that the six minimal squares are products of exactly five minimal abelian squares (this is straightforward to verify directly). Thus if $w$ is an optimal squareful word, then $\sqrt{w}=\sqrt[a b]{w}$. Thus by Theorem 3.2 the abelian square root of a Sturmian word $s_{x, \alpha}$ is the Sturmian word $s_{\psi(x), \alpha}$. Also, by Theorem 8.7 there exists a minimal subshift $\Omega$ such that for all $w \in \Omega$ either $\sqrt[a b]{w} \in \Omega$ or $\sqrt[a b]{w}$ is periodic. Saari proves in [17, Theorem 19] that an optimal abelian squareful word must have at least five distinct minimal abelian squares, but he leaves the characterization of these sets of minimal abelian squares open. Thus it is possible that there exists optimal abelian squareful words which contain other minimal abelian squares than those given by [17, Theorem 18]. For such words the abelian square root map could exhibit different behavior than the square root map (if the square root map is even defined for such words). We have not extended our research to this direction.

We could also generalize the special function $\psi$. Divide the distance $D$ between $x$ and $1-\alpha$ into $k$ parts and choose the image of $x$ to be $x+\frac{t}{k} D$ among the points

$$
x+\frac{1}{k} D, x+\frac{2}{k} D, \ldots, x+\frac{k-1}{k} D
$$

to obtain the function

$$
\psi_{k, t}: \mathbb{T} \rightarrow \mathbb{T}, x \mapsto \frac{1}{k}(t x+(k-t)(1-\alpha)) .
$$

The map $\psi_{k, t}$ is a perfectly nice function on the circle $\mathbb{T}$, but to make things interesting we would need to find a symbolic interpretation for it. We have not figured out any such interpretation for these generalized functions.

\section{Open Problems}

In the Section 8 we saw that there are non-Sturmian words whose language is preserved under the square root map. However, Sturmian words satisfy an even stronger property: by Theorem 3.2 for the Sturmian subshift $\Omega_{\alpha}$ of slope $\alpha$ it holds that $\sqrt{\Omega_{\alpha}} \subseteq \Omega_{\alpha}$. This property is not satisfied by the aperiodic and minimal subshift $\Omega_{\Gamma}$ of the word $\Gamma$ constructed in Section 8 since by Theorem 8.7 there is a word in $\Omega_{\Gamma}$ having periodic square root; since $\Omega_{\Gamma}$ is aperiodic and minimal, it cannot contain such words. We are thus led to ask the following question we could not answer:

Question. If $\Omega$ is a subshift containing optimal squareful words satisfying $\sqrt{\Omega} \subseteq \Omega$, does the subshift $\Omega$ only contain Sturmian words?

Let us briefly see that if we do not require all words in $\Omega$ to be aperiodic then the above question has a negative answer.

Proposition 10.1. There exists a non-minimal non-Sturmian subshift $\Omega$ containing squareful words such that $\sqrt{\Omega} \subseteq \Omega$.

Proof Sketch. Let $S$ be a seed solution as in Section 8, and let $\Gamma$ be a corresponding fixed point of the square root map generated by the seed $S$ as in Section 8 . Further, set $\Delta=S^{\omega}$, let $\Omega_{\Delta}$ be the subshift generated by $\Delta$, and let $\Omega_{\Gamma}$ be the subshift generated by $\Gamma$. If $w \in \Omega_{\Gamma}$, then by Theorem 8.7 either $\sqrt{w} \in \Omega_{\Gamma}$ or $\sqrt{w} \in \Omega_{\Delta}$. Hence if we are able to show that $\sqrt{\Omega_{\Delta}} \subseteq \Omega_{\Delta}$, then the non-minimal and non-Sturmian subshift $\Omega_{\Gamma} \cup \Omega_{\Delta}$ has the desired properties.

Let $w \in \Omega_{\Delta}$, so $w=T^{\ell}(\Delta)$ for some $0 \leq \ell<|S|$. Write $w$ as a product of minimal squares: $w=X_{1}^{2} X_{2}^{2} \cdots$. We can now argue as in the proof of Theorem 8.7. If $\left|X_{1}^{2} \cdots X_{n}^{2}\right|=|S|-\ell$ for some $n \geq 1$ or $\left|X_{1}^{2} \cdots X_{m}^{2}\right|=\left|S^{2}\right|-\ell$ for some $m \geq 1$, then using the fact that $\sqrt{\Delta}=\Delta$ it is 
straightforward to see that $\sqrt{w} \in \Omega_{\Delta}$. Otherwise either $\ell$ is a nicely repetitive position of $S$ or $\ell+\left|X_{1}^{2} \cdots X_{i}^{2}\right|-|S|$ is a nicely repetitive position of $S$ where

$$
i=\max \left\{j \in\{1,2, \ldots\}:\left|X_{1}^{2} \cdots X_{j}^{2}\right| \leq\left|S^{2}\right|-\ell\right\} .
$$

In both of these cases we deduce with the help of Lemma 8.10 that $\sqrt{w} \in \Omega_{\Delta}$.

There are other interesting related questions. Consider the limit set

$$
\Omega \cap \sqrt{\Omega} \cap \sqrt{\sqrt{\Omega}} \cap \ldots
$$

We know very little about the limit set except in the Sturmian case when it contains the two fixed points $01 c_{\alpha}$ and $10 c_{\alpha}$. For the word $\Gamma$ of Section 8 we proved that the limit set contains at least two fixed points. We ask:

Question. When is the limit set nonempty? If it is nonempty, does it always contain fixed points? Can it contain points which are not fixed points?

It is a genuine possibility that the limit set is empty. Consider for instance the word $\zeta=$ $\tau\left(\sigma^{\omega}(6)\right)$, the morphic image of the fixed point of the morphism $\sigma: 6 \mapsto 656556,5 \mapsto 5$ under $\tau: 6 \mapsto S_{6}^{2}, 5 \mapsto S_{5}^{2}$ where $S_{5}=100$ and $S_{6}=10010$ are minimal square roots of slope $\alpha=$ $[0 ; 2,1, \ldots]$. It is straightforward to verify that $\zeta$ is optimal squareful and uniformly recurrent and that the returns to the factor 101 in $\mathcal{L}(\zeta)$ are $10100,101(001)^{2} 00$ and $101(001)^{4} 00$. By considering all possible occurrences of the factor $w=\tau(56565) \in \mathcal{L}(\zeta)$ in any product of minimal squares of slope $\alpha$, it can be shown that the square root of the product always contains a return to the factor 101 which is not in $\mathcal{L}(\zeta)$. Since the factor $w$ occurs in every point in the subshift $\Omega_{\zeta}$ generated by $\zeta$, we conclude that $\Omega_{\zeta} \cap \sqrt{\Omega_{\zeta}}=\varnothing$.

In Section 8 we constructed infinite families of primitive solutions to (8) using the recurrence $\gamma_{k+1}=L\left(\gamma_{k}\right) \gamma_{k}^{2}$. Why this construction worked was because the seed solution $S$ and the word $L=L(S)$ satisfy $\sqrt{S S}=S, \sqrt{S L}=S, \sqrt{L S}=L$, and $\sqrt{L L}=L$, that is, $\sqrt{(L S S)^{2}}=\sqrt{L S \cdot S L \cdot S S}=L S S$. Similarly $\sqrt{(S L L L L)^{2}}=S L L L L$, so substituting for example $S=01010010$ we obtain the primitive solution

$$
S_{2} S_{1} S_{4} S_{3} S_{5} S_{4} S_{3} S_{5} S_{6} S_{5} S_{4} S_{3} S_{5} S_{4} S_{3}=0101001010010010100100101001001010010010
$$

to $(8)$ in $\mathcal{L}(1,0)$. More solutions can be obtained with analogous constructions. Restricting to the languages of optimal squareful words, we ask:

Question. What are the primitive solutions $w$ of $(8)$ in $\mathcal{L}(a, b)$ such that $w$ or $w^{2}$ is not Sturmian and $w$ is not obtainable by the above construction?

\section{Acknowledgments}

The authors were supported by University of Turku Graduate School UTUGS Matti programme and by the FiDiPro grant (137991) from the Academy of Finland.

We thank our supervisors Juhani Karhumäki and Luca Zamboni for suggesting that the square root map might preserve the language of a Sturmian word. We also thank Tero Harju for valuable comments.

\section{References}

[1] J. Berstel. On the index of Sturmian words. In: Jewels Are Forever. Springer-Verlag, 1999, pp. 287-294. 
[2] J. W. S. Cassels. An Introduction to Diophantine Approximation. Cambridge Tracts in Mathematics and Mathematical Physics 45. Cambridge University Press, 1957.

[3] D. Damanik and D. Lenz. Powers in Sturmian sequences. European Journal of Combinatorics 24 (2003), 377-390. DOI: 10.1016/S0195-6698(03)00026-X.

[4] A. De Luca and G. Fici. Open and closed prefixes of Sturmian words. Combinatorics on Words. 9th International Conference, WORDS 2013. Ed. by J. Karhumäki, A. Lepistö, and L. Zamboni. Lecture Notes in Computer Science 8079. Springer, 2013, pp. 132-142. DOI: 10.1007/978-3-642-40579-2.

[5] F. Durand, B. Host, and C. Skau. Substitution dynamical systems, Bratteli diagrams and dimension groups. Ergodic Theory and Dynamical Systems 19 (1999), 953-993.

[6] Štěpán Holub. A solution of the equation $\left(x_{1}^{2} \cdots x_{n}^{2}\right)^{3}=\left(x_{1}^{3} \cdots x_{n}^{3}\right)^{2}$. In: Contributions to General Algebra, 11 (Olomouc/Velké Karlovice, 1998). Heyn, Klagenfurt, 1999, pp. 105-111.

[7] Štěpán Holub. In search of a word with special combinatorial properties. In: Computational and Geometric Aspects of Modern Algebra. Vol. 275. London Mathematical Society Lecture Note Series. Cambridge University Press, 2000, pp. 120-127. DOI: 10.1017/CB09780511600609.011.

[8] Štěpán Holub. Local and global cyclicity in free semigroups. Theoretical Computer Science 262.1-2 (2001), 25-36. DOI: 10.1016/S0304-3975 (00)00156-0.

[9] A. Ya. Khinchin. Continued Fractions. Dover Publications, Mineola, New York, 1997.

[10] M. Lothaire. Combinatorics on Words. Encyclopedia of Mathematics and Its Applications 17. AddisonWesley, 1983.

[11] M. Lothaire. Algebraic Combinatorics on Words. Encyclopedia of Mathematics and Its Applications 90. Cambridge University Press, 2002.

[12] J. Peltomäki. Characterization of repetitions in Sturmian words: A new proof. Information Processing Letters 115.11 (2015), 886-891. DOI: 10.1016/j.ipl.2015.05.011.

[13] J. Peltomäki and M. Whiteland. A square root map on Sturmian words. (Extended abstract). Combinatorics of Words. 10th International Conference, WORDS 2015. Ed. by F. Manea and D. Nowotka. Lecture Notes in Computer Science 9304. Springer, 2015, pp. 197-209. DOI: 10. 1007/978-3-319-23660-5.

[14] N. Pytheas Fogg. Substitutions in Dynamics, Arithmetics and Combinatorics. Lecture Notes in Mathematics 1794. Springer, 2002. DOI: 10.1007/b13861.

[15] G. Richomme, K. Saari, and L. Q. Zamboni. Abelian complexity of minimal subshifts. Journal of the London Mathematical Society 83.2 (2011), 79-95. DOI: 10.1112/jlms/jdq063.

[16] K. Saari. “On the Frequency and Periodicity of Infinite Words". Ph.D. dissertation. Turku Centre for Computer Science, 2008. URL: http://users . utu.fi/kasaar/pubs/phdth.pdf.

[17] K. Saari. Everywhere $\alpha$-repetitive sequences and Sturmian words. European Journal of Combinatorics 31 (2010), 177-192. DOI: 10.1016/j . ejc. 2009.01.004. 